\title{
Pacific
}

Journal of

Mathematics

\section{THE BERGMAN AND SZEGŐ KERNELS NEAR POINTS OF INFINITE TYPE}

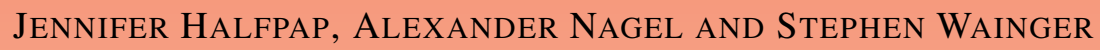




\title{
THE BERGMAN AND SZEGŐ KERNELS NEAR POINTS OF INFINITE TYPE
}

\author{
Jennifer HalfPap, Alexander Nagel And Stephen Wainger
}

\begin{abstract}
We study the singularities of the Bergman and Szegó kernels for domains $\Omega=\left\{\left(z_{1}, z_{2}\right) \in \mathbb{C}^{2} \mid \operatorname{Im} z_{2}>b\left(\operatorname{Re} z_{1}\right)\right\}$. Here $b$ is an even function in $C^{\infty}(\mathbb{R})$ satisfying $b(0)=b^{\prime}(0)=0, b^{\prime \prime}(r)>0$ for $r \neq 0$, and vanishing to infinite order at $r=0$. A model example is $b(r)=\exp \left(-|r|^{-a}\right)$ for $|r|$ small and $b(r)=r^{2 m}$ for $|r|$ large, with $a, m>0$. If $\Delta \subset \partial \Omega \times \partial \Omega$ is the diagonal of the boundary, our results show in particular that if $0<a<1$ the Bergman and Szegó kernels extend smoothly to $\bar{\Omega} \times \bar{\Omega} \backslash \Delta$, while if $a \geq 1$ the kernels are singular at points on $\bar{\Omega} \times \bar{\Omega} \backslash \Delta$.
\end{abstract}

\section{Introduction}

If $\Omega \subset \mathbb{C}^{n}$ is a domain, let $O(\Omega)$ denote the space of holomorphic functions on $\Omega$. The associated Bergman projection $\mathbf{B}=\mathbf{B}_{\Omega}$ is the orthogonal projection of $L^{2}(\Omega)$ onto the closed subspace $B^{2}(\Omega)=L^{2}(\Omega) \cap \mathcal{O}(\Omega)$ of square-integrable holomorphic functions on $\boldsymbol{\Omega}$. If $\boldsymbol{\Omega}$ has smooth boundary $\partial \boldsymbol{\Omega}$, the Szegő projection $\mathbf{S}=\mathbf{S}_{\boldsymbol{\Omega}}$ is the orthogonal projection of $L^{2}(\partial \Omega)$ onto the closed subspace $H^{2}(\Omega)$ of squareintegrable boundary values of holomorphic functions. These operators have integral representations

$$
\begin{aligned}
& \mathbf{B}[f](z)=\int_{\Omega} \mathscr{B}(z, w) f(w) d w, \\
& \mathbf{S}[f](z)=\int_{\partial \Omega} \mathscr{S}(z, w) f(w) d \sigma(w),
\end{aligned}
$$

where $d w$ denotes Lebesgue measure on $\Omega$ and $d \sigma$ is an appropriate measure on $\partial \Omega$. The functions $\mathscr{B}$ and $\mathscr{Y}$ are known respectively as the Bergman and Szegó kernels. In this paper we study the boundary behavior of these kernels on model domains for which the boundary has infinitely flat points but does not contain any analytic disks. Our main result is that in certain cases these kernels have singularities on the boundary of the domain away from the diagonal of the boundary.

MSC2000: 32T99, 42B20.

Keywords: Bergman kernel, Szegő kernel.

Halfpap was supported in part by NSF grant DMS-0654195 at the University of Montana. Wainger was supported in part by NSF grant DMS-0555850 at the University of Wisconsin-Madison. 
Explicitly, let $b \in \mathscr{C}^{\infty}(\mathbb{R})$, and put

$$
\Omega=\left\{\left(z_{1}, z_{2}\right) \in \mathbb{C}^{2} \mid \Im \mathrm{m}\left(z_{2}\right)>b\left(\Re \mathrm{e}\left(z_{1}\right)\right)\right\} .
$$

Then $\Omega$ is pseudoconvex if and only if $b$ is convex, $\Omega$ is of finite type if and only if $b^{\prime \prime}(r)$ vanishes to at most finite order at any point $r \in \mathbb{R}$, and $\partial \Omega$ contains an analytic disk if and only if $b^{\prime \prime}(r)=0$ on some nonempty open interval $I \subset \mathbb{R}$. In this paper, we consider the case in which $b$ is convex and even, and $b^{\prime \prime}(r)$ vanishes to infinite order at the point $r=0$ but is nonzero at all other points. We assume that for large $|r|$ the function $b$ behaves like a convex polynomial. The motivating examples for our analysis are the functions $b$ given for small $|r|$ by

$$
b(r)=\exp \left(-|r|^{-a}\right)
$$

for $a>0$. In this case, the behavior of the kernels $\mathscr{B}$ and $\mathscr{S}$ on $\partial \Omega \times \partial \Omega$ depends on the size of $a$. As particular examples of our main results, we show

(i) if $0<a<1$, the Bergman and Szegó kernels for $\Omega$ extend smoothly to $\bar{\Omega} \times \bar{\Omega} \backslash \Delta$, where $\Delta=\{(z, w) \in \partial \Omega \times \partial \boldsymbol{\Omega} \mid z=w\}$ is the diagonal of the boundary;

(ii) if $a>1$, the kernels are singular precisely at all points of $\Delta \cup \Sigma \subset \partial \Omega \times \partial \Omega$ where

$$
\Sigma=\left\{(z, w) \in \partial \Omega \times \partial \Omega \mid \Re \mathrm{e}\left(z_{1}\right)=\Re \mathrm{e}\left(w_{1}\right)=0, \Re \mathrm{e}\left(z_{2}\right)=\Re \mathrm{e}\left(w_{2}\right)\right\} ;
$$

(iii) if $a=1$, there is a constant $\epsilon>0$ such that the kernels are singular at all points of $\Delta \cup \Sigma_{\epsilon}$, where

$$
\Sigma_{\epsilon}=\left\{(z, w) \in \Sigma|| \mathfrak{\Im m}\left(z_{1}-w_{1}\right) \mid<\epsilon\right\} .
$$

1.1. Previous results. There is a huge literature on the boundary behavior of the Bergman kernel. Here we recall some results dealing with the smooth extension of the kernel $\mathscr{B}(z, w)$ to the boundary of $\Omega \times \Omega$.

First, it is known in many cases that the Bergman kernel for $\Omega$ becomes infinite on the diagonal $\Delta$ of the boundary $\partial \Omega$. If $z \in \Omega$, let $\delta(z)$ be the distance from $z$ to the boundary $\partial \Omega$. If $\Omega$ is strictly pseudoconvex, Hörmander [1965] showed that $\delta(z)^{n+1} \mathscr{B}(z, z)$ has a finite nonzero limit as $z \rightarrow z_{0} \in \partial \Omega$, and hence $\mathscr{B}(z, z) \rightarrow$ $+\infty$. In [Boas et al. 1995], Boas, Straube, and Yu established the existence of a finite limit for $\delta(z)^{m} B(z, z)$ for a much more general class of domains. Here $m$ is a constant that depends on certain boundary invariants of the domain at the boundary point $z_{0}$. More generally, Pflug $\left[1975\right.$; 1982] showed that if $\Omega \subset \mathbb{C}^{n}$ is pseudoconvex and has a $\mathscr{C}^{2}$-boundary, then $\mathscr{B}(z, z) \geq C_{\epsilon} \delta(z)^{-2+\epsilon}$ for any $\epsilon>0$. This result was later improved by Ohsawa [1984]. In [1994], Siqi Fu showed that Pflug's result holds with $\epsilon=0$. 
One can understand the singularity of the Bergman kernel on the diagonal $\Delta$ in terms of the existence of unbounded functions in $B^{2}(\Omega)$. Thus it is well known (see for example [Krantz 1992]) that the Bergman kernel on the diagonal solves an extremal problem: If $z \in \Omega$, then

$$
\mathscr{B}(z, z)=\sup \left\{|f(z)|^{2} \mid f \in B^{2}(\Omega) \text { and }\|f\|_{L^{2}(\Omega)} \leq 1\right\} .
$$

Thus if $\Omega \subset \mathbb{C}^{n}$ is a domain, if $z_{0} \in \partial \Omega$, and if there exists a square-integrable holomorphic function $f$ such that $\limsup _{z \rightarrow z_{0}}|f(z)|=+\infty$, then the Bergman kernel is singular at $\left(z_{0}, z_{0}\right)$.

On the other hand, it is sometimes possible to prove that the Bergman kernel extends smoothly to part of $\bar{\Omega} \times \bar{\Omega}$. Kerzman [1972] showed that if $\Omega \subset \mathbb{C}^{n}$ is a bounded strictly pseudoconvex domain, then the Bergman kernel $\mathscr{B}(z, w)$ extends to a $\mathscr{C}^{\infty}$-function on $\overline{\boldsymbol{\Omega}} \times \overline{\boldsymbol{\Omega}} \backslash \Delta$. Kerzman's argument uses J. J. Kohn's result on the existence of subelliptic estimates for the $\bar{\partial}$-Neumann problem in strictly pseudoconvex domains; see [Kohn 1963; 1964]. Later work of Kohn [1979], Catlin [1987], and Diederich and Fornaess [1978] established the existence of subelliptic estimates for bounded weakly pseudoconvex domains of finite type. Kerzman's result is true for these domains as well. Other results on the smooth extension of the Bergman kernel can be found in the work of Bell [1986] and Boas [1987]. For weakly pseudoconvex domains of finite type, the Bergman kernel extends to a $\mathscr{C}^{\infty}$ function precisely on the set $\overline{\boldsymbol{\Omega}} \times \overline{\boldsymbol{\Omega}} \backslash \Delta$.

If the domain $\Omega$ is not of finite type, the situation is less clear. For example, if the boundary $\partial \Omega$ contains an analytic disk, the singularities of the Bergman and Szegő kernels can "propagate" along the disk, and thus the kernels can have singularities away from the diagonal. A simple example is the polydisk in $\mathbb{C}^{n}$, where the Bergman and Szegó kernels are just products of one-dimensional kernels in each variable. The results of this paper show that the Bergman and Szegó kernels can be singular away from the diagonal of the boundary even if there are no analytic disks in the boundary.

When $b$ is a convex polynomial, domains of the form given in (1-3) were discussed in [Nagel 1986], which gives an explicit integral formula for the Szegó kernel:

$$
\mathscr{Y}\left(\left(z_{1}, z_{2}\right),\left(w_{1}, w_{2}\right)\right)=\frac{1}{2 \pi^{2}} \iint_{\tau>0} \frac{e^{\eta\left(z_{1}+\bar{w}_{1}\right)+i \tau\left(z_{2}-\bar{w}_{2}\right)}}{\int_{\mathbb{R}} e^{2(\eta r-\tau b(r))} d r} d \eta d \tau .
$$

That paper estimates this integral, and shows that there is a natural nonisotropic metric on $\partial \Omega$ relative to which the Szegő projection is a variant of a classical Calderón-Zygmund operator. The integral formula (1-8) was later used by Christ and Geller [1992] to obtain examples of the failure of analytic hypoellipticity in domains of finite type. Haslinger [1995] considered such integrals, and then 
Christ [2002] made a deeper study of this formula. ${ }^{1}$ The program of studying the Szegó projection $\mathbf{S}$ as a singular integral operator was carried out for general pseudoconvex domains of finite type in $\mathbb{C}^{2}$ in [Nagel et al. 1988; 1989].

There are closely related problems involving the functions $b(r)=\exp \left(-|r|^{-a}\right)$ for which there is a qualitative difference in behavior for $a<1$ and for $a \geq 1$. For example, Kusuoka and Stroock [1985] showed that the second order partial differential operator

$$
L=\frac{\partial^{2}}{\partial x^{2}}+\frac{\partial^{2}}{\partial y^{2}}+e^{-|x|^{-a}} \frac{\partial^{2}}{\partial t^{2}}
$$

is hypoelliptic if and only if $a<1$. This result has led to considerable further research; see for example [Christ 2001a; 2001b].

1.2. Plan of the paper. The double integral in (1-8) involves two oscillatory terms: $e^{i \eta \Im \mathrm{m}\left(z_{1}+\bar{w}_{1}\right)}$ and $e^{i \tau \Re \mathrm{Re}\left(z_{2}-\bar{w}_{2}\right)}$. To obtain good estimates for the integral, one needs to take advantage of these oscillations. When $b$ is a polynomial, this is accomplished in [Nagel 1986] essentially through integration by parts. However, if $b^{\prime \prime}(r)$ vanishes to infinite order, it seems that integration by parts in $\eta$ no longer suffices to give good estimates. To overcome this difficulty, we will regard the function

$$
N(\eta, \tau)=\int_{-\infty}^{+\infty} e^{2(\eta r-\tau b(r))} d r
$$

as a holomorphic function of $\eta, \tau \in \mathbb{C}$ with $\Re \mathrm{e}(\tau)>0$. We can then use Cauchy's theorem to change a contour in (1-8). Since the function $N(\eta, \tau)$ appears in the denominator, we need information about the location of its zeros. (This is also a main concern of [Christ and Geller 1992] and [Christ 2002]). In particular, to show that for $a \geq 1$ in (1-4), the kernels are singular off the diagonal of the boundary, we show that the main contribution to the size of the kernel comes from the residue of $N(\eta, \tau)^{-1}$ at the smallest zero on the imaginary axis.

The rest of the paper is organized as follows.

In Section 2, we give precise definitions of the Szegó projection and a family of Bergman projections in weighted $L^{2}$-spaces. The domain $\Omega$ is unbounded, and we take this opportunity to show how the usual theory for bounded domains carries over to this situation.

In Section 3, we introduce appropriate notation and state our basic results. Theorem 3.4 gives formulas for the Bergman and Szegó kernels. Theorem 3.6 shows that the kernels extend to $\mathscr{C}^{\infty}$-functions on appropriate parts of the boundary. Theorem 3.8 shows that the kernels are singular on the set $\Sigma$ defined in (1-5).

The proofs of Theorems 3.4 and 3.6 depend on various estimates for integrals involving convex functions. Rather than prove these results piecemeal, we gather

\footnotetext{
${ }^{1}$ See especially [Christ 2002, Lemma 4].
} 
these technical arguments together in Section 4. In particular, the change in the contour of integration in (1-8) leads to the integrals in Lemmas 4.17 and 4.18. These are the key estimates of the paper. To deal with the integrals we need very precise estimates for the functions $\varphi(\lambda, \tau)$ and $h_{\alpha}(\lambda-\alpha)$ that appear there, and these in turn require the results in the earlier parts of the section.

In Section 5, we study the function $N(\eta, \tau)$ defined in (1-10) as a function of complex variables.

Finally, in Sections 6, 7 and 8 we give the proofs of Theorems 3.4, 3.6, and 3.8.

\section{Bergman and Szegő projections}

The domain $\Omega$ given in (1-3) is unbounded, but most standard discussions of Bergman and Szegó projections deal with the case of bounded domains. In this section we give precise definitions of the relevant weighted Bergman spaces $B_{\rho}^{2}(\Omega)$ and the space $\mathscr{H}^{2}(\Omega)$, and develop enough of the theory in our unbounded context to allow us to derive formulas such as (1-8) for the Bergman and Szegó projections.

Let $b \in \mathscr{C}^{\infty}(\mathbb{R})$, and suppose $b^{\prime \prime}(r) \geq 0, b(r)=b(-r)$, and $b(0)=b^{\prime}(0)=0$. As in (1-3), put

$\Omega=\left\{\left(z_{1}, z_{2}\right) \in \mathbb{C}^{2} \mid \Im \mathrm{m}\left(z_{2}\right)>b\left(\Re \mathrm{e}\left(z_{1}\right)\right)\right\}=\left\{(x+i y, t+i s) \in \mathbb{C}^{2} \mid b(x)-s<0\right\}$.

We identify the boundary of $\Omega$ with $\mathbb{R}^{3}$ so that $(x+i y, t+i b(x)) \in \partial \Omega$ corresponds to $(x, y, t) \in \mathbb{R}^{3}$. We take Lebesgue measure $d x d y d t$ as the measure on the boundary. ${ }^{2}$

2.1. Weighted Bergman spaces and Bergman projections. For $0 \leq \rho<1$, let $d m_{\rho}$ denote the measure on $\Omega$ given by $(s-b(x))^{-\rho} d x d y d t d s$, and let $L_{\rho}^{2}(\Omega)$ denote the space of Lebesgue measurable functions on $\Omega$ that are square integrable with respect to the measure $d m_{\rho}$. Note that $s-b(x)$ is essentially the distance to the boundary. The norm in $L_{\rho}^{2}(\Omega)$ is given by

$$
\|f\|_{\rho}^{2}=\int_{\Omega}|f(x, y, t, s)|^{2}(s-b(x))^{-\rho} d x d y d t d s .
$$

The weighted Bergman space for the measure $d m_{\rho}$ is

$$
B_{\rho}^{2}(\Omega)=\left\{F \in \mathcal{O}(\Omega) \mid F \in L_{\rho}^{2}(\Omega)\right\} .
$$

The mean-value property of holomorphic functions implies that for any compact set $K \subset \Omega$, there is a constant $C_{K}$ such that $\sup _{z \in K}|f(z)| \leq C_{K}\|f\|_{\rho}$ if $f \in B_{\rho}^{2}(\Omega)$.

${ }^{2}$ Lebesgue measure is not the same as the surface area measure $d \sigma_{s}$ on the boundary of $\Omega$; in fact, $d \sigma_{S}=\sqrt{1+b^{\prime}(x)^{2}} d x d y d t$. Since $\sqrt{1+b^{\prime}(x)^{2}}$ is unbounded, using this measure would result in a different space of boundary values. We choose $d x d y d t$ because we want $\mathscr{H}^{2}(\Omega)$ to be the limiting case as $\rho \rightarrow 1$ of the spaces $B_{\rho}^{2}(\Omega)$ defined in Section 2.1 . 
It follows that $B_{\rho}^{2}(\Omega)$ is a closed subspace of $L_{\rho}^{2}(\Omega)$, and the Bergman projection is the orthogonal projection

$$
\mathbf{B}_{\rho}: L_{\rho}^{2}(\Omega) \rightarrow B_{\rho}^{2}(\Omega) .
$$

The case $\rho=0$ is the standard Bergman space.

The operator $\mathbf{B}_{\rho}$ is given by integration against the Bergman kernel $\mathscr{B}_{\rho}(z, w)$, which is defined as follows. Point evaluation $f \rightarrow f(z)$ is a bounded linear functional on $B_{\rho}^{2}(\Omega)$ and hence by the Riesz representation theorem is given by integrating $f(w)$ against the conjugate of an element $b_{z}(w)$ in $B_{\rho}^{2}(\Omega)$. Then $\mathscr{B}_{\rho}(z, w)=\overline{b_{z}(w)}$. The following result is standard; see, e.g., [Krantz 1992].

Proposition 2.2. There exists a unique function $\mathscr{B}_{\rho}: \Omega \times \Omega \rightarrow \mathbb{C}$, called the Bergman kernel, such that

(a) for each $w \in \Omega$, the function $z \rightarrow \mathscr{B}_{\rho}(z, w)$ belongs to $B_{\rho}^{2}(\Omega)$;

(b) $\mathscr{B}_{\rho}(z, w)=\overline{\mathscr{B}_{\rho}(w, z)}$ for $z, w \in \Omega$;

(c) $f(z)=\int_{\Omega} \mathscr{B}_{\rho}(z, w) f(w) d m_{\rho}(w)$ for $f \in B_{\rho}(\Omega)$;

(d) $\mathbf{B}_{\rho}[f](z)=\int_{\Omega} \mathscr{B}_{\rho}(z, w) f(w) d m_{\rho}(w)$ for $f \in L_{\rho}^{2}(\Omega)$.

2.3. The space $\mathscr{H}^{2}(\Omega)$ and the Szegó projection. In an appropriate sense, the limiting case of $B_{\rho}^{2}(\Omega)$ as $\rho \rightarrow 1$ is the Hardy space $\mathcal{H}^{2}(\Omega)$, but dealing with boundary values that are defined only almost everywhere makes its definition more delicate than that of $B_{\rho}^{2}(\Omega){ }^{3}$ We proceed as follows. For $F \in \mathbb{O}(\Omega)$ and $\epsilon>0$, set

$$
F_{\epsilon}(x, y, t)=F(x+i y, t+i b(x)+i \epsilon) .
$$

Then

$$
\mathscr{H}^{2}(\Omega)=\left\{\left.F \in \mathcal{O}(\Omega)\left|\sup _{\epsilon>0} \int_{\partial \Omega}\right| F_{\epsilon}(x, y, t)\right|^{2} d x d y d t \equiv\|F\|_{\mathscr{H}^{2}}^{2}<\infty\right\} .
$$

The following proposition gives some of the basic facts about $\mathscr{H}^{2}(\Omega)$. In particular, it shows that $\mathscr{H}^{2}(\Omega)$ can be identified with a closed subspace of $L^{2}(\partial \Omega)=L^{2}\left(\mathbb{R}^{3}\right)$.

Proposition 2.4. Let $F \in \mathscr{H}^{2}(\Omega)$. Then there exists $F^{b} \in L^{2}(\partial \Omega)$ such that

(a) $F^{b}(x, y, t)=\lim _{\epsilon \rightarrow 0^{+}} F_{\epsilon}(x, y, t)$ for almost every $(x, y, t) \in \mathbb{R}^{3}$;

(b) there is a constant $C$ independent of $F$ such that

$$
\int_{\mathbb{R}^{3}} \sup _{s>0}|F(x+i y, t+i b(x)+i s)|^{2} d x d y d t \leq C^{2}\|F\|_{\mathscr{H}^{2}}^{2} ;
$$

${ }^{3} \mathrm{~A}$ discussion of the Bergman and Hardy spaces on certain model domains can be found [Haslinger 1998]. 
(c) $\lim _{\epsilon \rightarrow 0^{+}}\left\|F_{\epsilon}-F^{b}\right\|_{L^{2}(\partial \Omega)}=0$, and $\left\|F^{b}\right\|_{L^{2}\left(\mathbb{R}^{3}\right)}=\|F\|_{\mathscr{L}^{2}(\Omega)}$;

(d) the boundary function $F^{b}$ satisfies the differential equation

$$
\frac{\partial F^{b}}{\partial x}+i \frac{\partial F^{b}}{\partial y}-i b^{\prime}(x) \frac{\partial F^{b}}{\partial t}=0
$$

in the sense of distributions;

(e) for any compact subset $K \subset \Omega$, there is a positive constant $C(K)$ independent of $F$ such that

$$
\sup _{z \in K}|F(z)| \leq C(K)\|F\|_{\mathscr{H}^{2}} .
$$

Proof. Parts (a), (b), and (c) follow as in [Stein 1993] from the analogous results ${ }^{4}$ for the Hardy space $\mathscr{H}^{2}(U)$ in the upper half-space $U=\{z=x+i y \in \mathbb{C} \mid y>0\}$. Part (d) follows from (c) and the fact that each $F_{\epsilon}$ satisfies the differential equation of (d) in the classical sense since it is the restriction to $\partial \Omega$ of a holomorphic function defined in a neighborhood of $\partial \Omega$. Part (e) follows from the mean-value property of holomorphic functions and the estimate corresponding to that of (e) for $\mathcal{H}^{2}(U)$.

We shall also need the fact that the space of boundary values $F^{b} \in L^{2}(\partial \Omega)$ of functions $F \in \mathscr{H}^{2}(\Omega)$ is exactly the space of functions $f \in L^{2}(\partial \Omega)$ that satisfy the inequality in (b) in the sense of distributions. To see this, define a partial Fourier transform $\mathscr{F}: L^{2}\left(\mathbb{R}^{3}\right) \rightarrow L^{2}\left(\mathbb{R}^{3}\right)$ by the integral

$$
\mathscr{F}[f](x, \eta, \tau)=\hat{f}(x, \eta, \tau)=\iint_{\mathbb{R}^{2}} e^{-2 \pi i(y \eta+t \tau)} f(x, y, t) d y d t .
$$

This converges for almost every $x \in \mathbb{R}$ if $f \in L^{1}\left(\mathbb{R}^{3}\right)$. The two-variable Plancherel theorem and Fubini's theorem then give

$$
\iiint_{\mathbb{R}^{3}}|f(x, y, t)|^{2} d x d y d t=\iiint_{\mathbb{R}^{3}}|\hat{f}(x, \eta, \tau)|^{2} d x d \eta d \tau
$$

if $f \in L^{1}\left(\mathbb{R}^{3}\right) \cap L^{2}\left(\mathbb{R}^{3}\right)$. Thus $\mathscr{F}$ extends to an isometry of $L^{2}\left(\mathbb{R}^{3}\right)$. If $\hat{f} \in L^{1}\left(\mathbb{R}^{3}\right)$, the inversion formula

$$
f(x, y, t)=\iint_{\mathbb{R}^{2}} e^{2 \pi i(y \eta+t \tau)} \hat{f}(x, \eta, \tau) d \eta d \tau
$$

holds for almost every $x \in \mathbb{R}$.

Proposition 2.5. Let $b$ be convex and even, let $b(0)=b^{\prime}(0)=0$, and suppose $\lim _{x \rightarrow \pm \infty}|x|^{-1} b(x)=+\infty$. Let $f \in L^{2}(\partial \Omega)=L^{2}\left(\mathbb{R}^{3}\right)$. Then

\footnotetext{
${ }^{4}$ The argument in [Stein 1993] deals with the case in which there is a large group of biholomorphic mappings of $\Omega$, and this is not true in our situation. For convenience, we include the details of the argument in the appendix, along with a discussion of the spaces $\mathscr{H}^{p}(\Omega)$ for $1<p<\infty$. Only minor modifications of the argument in [Stein 1993] are needed.
} 
(a) the function $f$ satisfies

$$
\frac{\partial f}{\partial x}+i \frac{\partial f}{\partial y}-i b^{\prime}(x) \frac{\partial f}{\partial t}=0
$$

on $\mathbb{R}^{3}$ in the distributional sense if and only if the partial Fourier transform $\mathscr{F}[f]=\hat{f}$ satisfies

$$
\frac{\partial}{\partial x}\left(e^{-2 \pi(\eta x-\tau b(x))} \hat{f}(x, \eta, \tau)\right)=0
$$

on $\mathbb{R}^{3}$ in the sense of distributions;

(b) if $f$ satisfies (2-9), then $\hat{f}(x, \eta, \tau)=0$ almost everywhere when $\tau<0$. In particular, if we set $h_{s}(x, \eta, \tau)=e^{-2 \pi \tau s} \hat{f}(x, \eta, \tau)$, then $h_{s} \in L^{2}\left(\mathbb{R}^{3}\right)$ for $s \geq 0$

(c) if $f$ satisfies (2-9) and if

$$
F\left(z_{1}, z_{2}\right)=F(x+i y, t+i b(x)+i s)=\mathscr{F}^{-1}\left[h_{s}\right](x, y, t),
$$

then $F \in \mathscr{H}^{2}(\Omega)$ and $F^{b}=f$.

Proof. Part (a) follows directly from (2-6) if $f \in \mathscr{C}_{0}^{\infty}\left(\mathbb{R}^{3}\right)$. If $f \in L^{2}\left(\mathbb{R}^{3}\right)$, the equivalence of (2-9) and (2-10) follows from the identity

$$
\iiint_{\mathbb{R}^{3}} f_{1}(x, y, t) \overline{f_{2}(x, y, t)} d x d y d t=\iiint_{\mathbb{R}^{3}} \hat{f}_{1}(x, \eta, \tau) \overline{\hat{f}_{2}(x, \eta, \tau)} d x d \eta d \tau
$$

and integration by parts when $f_{1}, f_{2} \in L^{2}\left(\mathbb{R}^{3}\right)$ and $f_{2} \in \mathscr{C}_{0}^{\infty}\left(\mathbb{R}^{3}\right)$.

To establish (b), note that there is a set $E \subset \mathbb{R}^{2}$ of measure zero such that if $(\eta, \tau) \notin E$, the function $x \rightarrow \hat{f}(x, \eta, \tau)$ belongs to $L^{2}(\mathbb{R})$. For such $(\eta, \tau)$, it follows from (2-10) that $x \rightarrow e^{-2 \pi(\eta x-\tau b(x))} \hat{f}(x, \eta, \tau)$ is a constant, which we write $g(\eta, \tau)$. But then

$$
\iint|g(\eta, \tau)|^{2}\left(\int_{\mathbb{R}} e^{4 \pi(\eta x-\tau b(x))} d x\right) d \eta d \tau=\iiint_{\mathbb{R}^{3}}|\hat{f}(x, \eta, \tau)|^{2} d x d \eta d \tau<+\infty .
$$

It follows from the hypothesis $\lim _{x \rightarrow \pm \infty}|x|^{-1} b(x)=+\infty$ that the inner integral on the left side is infinite for $\tau<0$, and hence we must have $\hat{f}(x, \eta, \tau)=0$ almost everywhere for $\tau<0$. The statement about $h_{s}$ is then clear.

To establish (c), observe that Plancherel's theorem gives

$$
\begin{aligned}
\iiint_{\mathbb{R}^{3}}|F(x+i y, t+i b(x)+i s)|^{2} d x d y d t & =\iiint_{\mathbb{R}^{3}} e^{-4 \pi \tau s}|\hat{f}(x, \eta, \tau)| t^{2} d x d \eta d \tau \\
& \leq\|\hat{f}\|_{L^{2}}=\|f\|_{L^{2}} .
\end{aligned}
$$

Thus the function $F$ is locally square-integrable on $\Omega$. On the other hand, the inverse partial Fourier transform $\mathscr{F}^{-1}\left[h_{s}\right]$ is the limit in $L^{2}\left(\mathbb{R}^{3}\right)$ as $N \rightarrow \infty$ of the 
functions

$$
\begin{gathered}
\iint_{\eta^{2}+\tau^{2} \leq N^{2}} e^{2 \pi i[\eta y+\tau t]} h_{s}(x, \eta, \tau) d \eta d \tau=\iint_{\eta^{2}+\tau^{2} \leq N^{2}} e^{2 \pi i[\eta y+\tau t]} e^{-2 \pi \tau s} \hat{f}(x, \eta, \tau) d \eta d \tau \\
=\iint_{\eta^{2}+\tau^{2} \leq N^{2}} e^{2 \pi[\eta(x+i y)+i \tau(t+i b(x)+i s)]} g(\eta, \tau) d \eta d \tau \\
=\iiint_{\eta^{2}+\tau^{2} \leq N^{2}} e^{2 \pi\left[\eta z_{1}+i \tau z_{2}\right]} g(\eta, \tau) d \eta d \tau=F_{N}\left(z_{1}, z_{2}\right) .
\end{gathered}
$$

It is clear that each $F_{N}$ is holomorphic on $\Omega$, and since $F_{N} \rightarrow F$ locally in $L^{2}$, it follows that $F$ is also holomorphic. Thus $F \in \mathscr{H}^{2}(\Omega)$. Finally, it follows from Proposition 2.4 that $F$ has boundary values $F^{b}$ and $\lim _{s \rightarrow 0^{+}} F_{s}=F^{b}$ in $L^{2}\left(\mathbb{R}^{3}\right)$. However $F_{s}=\mathscr{F}^{-1}\left[h_{s}\right]$, and $\lim _{s \rightarrow 0^{+}} h_{s}=\hat{f}$ in $L^{2}\left(\mathbb{R}^{3}\right)$. Since $\mathscr{F}^{-1}$ is an isometry, it follows that $F^{b}=f$.

Corollary 2.6. The set of functions $f \in L^{2}(\partial \Omega)$ such that there exists $F \in \mathscr{H}^{2}(\Omega)$ with $F^{b}=f$ is the set of functions whose partial Fourier transforms $\hat{f}$ satisfy equation (2-10) in the sense of distributions.

We now define the Szegó projection $\mathbf{S}$ for the domain $\Omega$ to be the orthogonal projection from $L^{2}\left(\mathbb{R}^{3}\right)$ to the closed subspace of functions $f \in L^{2}\left(\mathbb{R}^{3}\right)$ satisfying $\partial f / \partial x+i \partial f / \partial y-i b^{\prime}(x) \partial f / \partial t=0$ in the sense of distributions. It follows from Corollary 2.6 that $\mathbf{S}$ maps $L^{2}(\partial \Omega)$ to $\mathscr{H}^{2}(\Omega)$.

\section{Main results}

3.1. Basic assumptions on the function $\boldsymbol{b}$. We make the following hypotheses about the function $b \in \mathscr{C}^{\infty}(\mathbb{R})$ used in the definition of the domain $\Omega$ given in Equation (1-3).

(3-1) $b$ is convex on $\mathbb{R}$ and is normalized at $0 \in \mathbb{R}$ :

$$
b^{\prime \prime}(r) \geq 0, \quad b(0)=0, \quad b^{\prime}(0)=0 .
$$

(3-2) $b$ is even: $b(r)=b(-r)$.

(3-3) $b^{\prime}$ and $b^{\prime \prime}$ are convex on the positive real axis: ${ }^{5}$

$$
r \geq 0 \text { implies } b^{(3)}(r) \geq 0 \text { and } b^{(4)}(r) \geq 0 \text {. }
$$

(3-4) Let $B(r)=\int_{0}^{r} b(t) d t$, so that $B^{\prime}(r)=b(r)$ and $B(0)=0$.

Then there exists a constant $C>1$ such that

$$
0<\frac{B^{(j+1)}(r)}{B^{(j)}(r)} \leq C \frac{B^{(k+1)}(r)}{B^{(k)}(r)} \quad \text { for } r>0 \text { and any } j, k \in\{0,1,2,3\},
$$


(3-5) There is an even convex polynomial $P$ of degree $N \geq 2$ such that

$$
|r| \geq 1 \text { implies } b(r)=P(r) .
$$

Suppose that $b$ satisfies (3-1)-(3-5). We introduce a quantity that measures the degree of "flatness" of the function $b$ at $r=0$. For $r \neq 0$, put

$$
\Theta(r)=b(r) /\left(r b^{\prime}(r)\right) .
$$

$\Theta$ is also an even function. Since $b^{\prime}$ is increasing on the positive real axis, we have $0<\Theta(r) \leq 1$ for $r \neq 0$. If $b$ is a convex polynomial, Bruna et. al. [1988] showed that there is an $\epsilon>0$ depending only on the degree such that $\epsilon \leq \Theta(r) \leq 1$ for $r \neq 0$.

In contrast, we are interested in the case that $b$ vanishes to infinite order at the origin and $\lim _{r \rightarrow 0} \Theta(r)=0$. If $b(r)=\exp \left(-|r|^{-a}\right)$ for small $|r|$ as in (1-4), then for these $r$ we have $b(r) /\left(r b^{\prime}(r)\right)=|r|^{a} / a$.

Definition 3.2. Let $b \in \mathscr{C}^{\infty}(\mathbb{R})$ satisfy (3-1)-(3-5), and define $\Theta$ as in (3-6). Then $b$ is subcritical at $r=0$ if there are constants $c>0$ and $0<\gamma<1$ such that

$$
\Theta(r)>c|r|^{\gamma} \quad \text { for small }|r|
$$

it is critical at $r=0$ if there is a constant $0<C<\infty$ such that

$$
\Theta(r) \leq C|r| \text { for small }|r|
$$

and it is supercritical at $r=0$ if there are constants $C>0$ and $a>1$ such that

$$
\Theta(r) \leq C|r|^{a} \text { for small }|r| \text {. }
$$

Throughout this paper, we shall use the symbol $A$ to denote a constant that is independent of the choice of the function $b$, while we shall use $C$ to denote a constant depending on the convex function $b$ but independent of other parameters. As usual, the value of $A$ or $C$ may change from line to line.

3.3. The Bergman and Szegó kernels: absolute convergence. We begin with the formulas for the integral kernels for the Bergman and Szegó projections. For $\eta \in \mathbb{R}$ and $\tau>0$, set

$$
N(\eta, \tau)=\int_{-\infty}^{+\infty} e^{2(\eta r-\tau b(r))} d r
$$

Then for $(z, w)=\left(\left(z_{1}, z_{2}\right),\left(w_{1}, w_{2}\right)\right) \in \mathbb{C}^{2} \times \mathbb{C}^{2}$ and $0 \leq \rho \leq 1$, set

$$
\mathscr{B}_{\rho}(z, w)=\frac{2^{1-\rho}}{4 \pi^{2} \Gamma(1-\rho)} \iint_{\tau>0} e^{\eta\left(z_{1}+\bar{w}_{1}\right)+i \tau\left(z_{2}-\bar{w}_{2}\right)} \frac{\tau^{1-\rho}}{N(\eta, \tau)} d \eta d \tau .
$$

\footnotetext{
${ }^{5}$ It is quite possible that weaker conditions would suffice.
} 
If we can show that the integral in (3-8) converges absolutely in a neighborhood of a point $\left(z_{0}, w_{0}\right) \in \mathbb{C}^{2} \times \mathbb{C}^{2}$, the function $\mathscr{B}_{\rho}(z, w)$ is holomorphic in $z$ and conjugate holomorphic in $w$ in that neighborhood. The first theorem describes such a region of absolute convergence, and identifies the functions $\mathscr{B}_{\rho}$ for $\rho \leq 1$ with the Szegô and Bergman kernels.

We use the notation

$$
\begin{aligned}
z=\left(z_{1}, z_{2}\right) & =(x+i y, t+i b(x)+i h), \\
w=\left(w_{1}, w_{2}\right) & =(u+i v, s+i b(u)+i k) .
\end{aligned}
$$

Theorem 3.4. Suppose $b \in \mathscr{C}^{\infty}(\mathbb{R})$ satisfies the hypotheses in (3-1)-(3-5).

(a) The integral in (3-8) defining $\mathscr{B}_{\rho}(z, w)$ converges absolutely in the region

$$
h+k+b(x)+b(u)-2 b\left(\frac{1}{2}(x+u)\right)>0 .
$$

This is an open neighborhood of the set $(\Omega \times \Omega) \cup\{(z, w) \in \bar{\Omega} \times \bar{\Omega} \mid x \neq u\}$. More generally, for any nonnegative integers $a, b, c, d$,

$$
\begin{aligned}
\partial_{z_{1}}^{a} \partial_{\bar{w}_{1}}^{b} \partial_{z_{2}}^{c} \partial_{\bar{w}_{2}} \mathscr{P}_{\rho}(z, w) & \\
& =\frac{2^{1-\rho} i^{c-d}}{4 \pi^{2} \Gamma(1-\rho)} \iint_{\tau>0} e^{\eta\left(z_{1}+\bar{w}_{1}\right)+i \tau\left(z_{2}-\bar{w}_{2}\right)} \frac{\eta^{a+b} \tau^{c+d+1-\rho}}{N(\eta, \tau)} d \eta d \tau,
\end{aligned}
$$

and this integral converges absolutely in the same region.

(b) The Bergman projection $\mathbf{B}_{\rho}$, defined in equation (2-3) for $\rho<1$, is given by

$$
\mathbf{B}_{\rho}[f](z)=\int_{\Omega} \mathscr{B}_{\rho}(z, w) f(w) d m_{\rho}(w) \quad \text { for } z \in \Omega .
$$

(c) The Szegó projection $\mathbf{S}$, defined after Corollary 2.6, is given by

$$
\mathbf{S}[f](z)=\int_{\partial \Omega} \mathscr{B}_{1}(z, w) f(w) d w \quad \text { for } z \in \Omega .
$$

\subsection{The Bergman and Szegö kernels: smooth extensions.}

Theorem 3.6. Suppose $b \in \mathscr{C}^{\infty}(\mathbb{R})$ satisfies the hypotheses in (3-1)-(3-5).

(a) Suppose b is subcritical. If $U$ is any open neighborhood in $\mathbb{C}^{4}$ of the diagonal $\Delta$ in $\partial \Omega \times \partial \Omega$, and if $a, b, c, d$ are arbitrary nonnegative integers, the function $\partial_{z_{1}}^{a} \partial_{\bar{w}_{1}}^{b} \partial_{z_{2}}^{c} \partial_{\bar{w}_{2}}^{d} \mathscr{P}_{\rho}(z, w)$ is uniformly bounded on $(\Omega \times \Omega) \backslash \mathcal{U}$. In particular, the function $\mathscr{B}_{\rho}$ extends to a $\mathscr{C}^{\infty}$-function on $(\bar{\Omega} \times \bar{\Omega}) \backslash \Delta$.

(b) Suppose b is supercritical. Let

$$
\Sigma=\{(z, w) \in \partial \Omega \times \partial \Omega \mid x=u=0, t=s\} .
$$


Let $\mathcal{W}$ be any open neighborhood in $\mathbb{C}^{4}$ of $\Delta \cup \Sigma$ in $\partial \Omega \times \partial \Omega$. If $a, b, c, d$ are arbitrary nonnegative integers, $\partial_{z_{1}}^{a} \partial_{\bar{w}_{1}}^{b} \partial_{z_{2}}^{c} \partial_{\bar{w}_{2}}^{d} \Re_{\rho}(z, w)$ is uniformly bounded on $(\Omega \times \Omega) \backslash \mathscr{W}$. In particular, the function $\mathscr{B}_{\rho}$ extends to a $\mathscr{C}^{\infty}$ function on $(\bar{\Omega} \times \bar{\Omega}) \backslash(\Delta \cup \Sigma)$.

3.7. The Bergman and Szegó kernels: singularities off the diagonal. The next results assert that in the critical or supercritical case, the kernels $\mathscr{B}_{\rho}$ for $0 \leq \rho \leq 1$ actually have singularities away from the diagonal.

Theorem 3.8. Suppose $b \in \mathscr{C}^{\infty}(\mathbb{R})$ satisfies the hypotheses in (3-1)-(3-5). Let $h, k>0$ and put $\delta=h+k$. Let $t \in \mathbb{R}$.

(a) If $b$ is critical, there exists $\epsilon>0$ such that if $|y-v|<\epsilon$,

$$
\lim _{\delta \rightarrow 0}\left|\Re_{\rho}((i y, t+i h),(i v, t+i k))\right|=+\infty .
$$

(b) If b is supercritical,

$$
\lim _{\delta \rightarrow 0}\left|\Re_{\rho}((i y, t+i h),(i v, t+i k))\right|=+\infty \quad \text { for all } y, v \in \mathbb{R} .
$$

\section{Estimates for convex functions}

In this section we establish various estimates that are needed for the proofs of Theorems 3.4, 3.6, and 3.8.

4.1. Notation. For any smooth convex function $b$ with $b(0)=b^{\prime}(0)=0$, define renormalizations about an arbitrary point $\alpha \in \mathbb{R}$, by setting

$$
b_{\alpha}(r)=b(\alpha+r)-b(\alpha)-r b^{\prime}(\alpha)=\int_{0}^{r} \int_{0}^{t} b^{\prime \prime}(\alpha+s) d s d t .
$$

Then

$$
\begin{aligned}
& b_{\alpha}^{\prime}(r)=b^{\prime}(\alpha+r)-b^{\prime}(\alpha)=\int_{0}^{r} b^{\prime \prime}(\alpha+t) d t, \quad \text { and } \\
& b_{\alpha}^{\prime \prime}(r)=b^{\prime \prime}(\alpha+r) \geq 0 .
\end{aligned}
$$

If $b$ satisfies (3-1), then each $b_{\alpha}$ also satisfies (3-1), and $b_{0}=b$. It is also easy to check that

$$
\left(b_{\alpha_{1}}\right)_{\alpha_{2}}=b_{\alpha_{1}+\alpha_{2}} .
$$

Next, given $b$, let $h$ denote the function ${ }^{6}$ given by

$$
h(r)=r b^{\prime}(r)-b(r) .
$$

${ }^{6}$ If $\hat{b}$ denotes the Legendre transform of $b$, then $h(r)=\hat{b}\left(\left(b^{\prime}\right)^{-1}(r)\right)$. 
Also put

$$
\begin{aligned}
h_{\alpha}(r)=r b_{\alpha}^{\prime}(r)-b_{\alpha}(r) & =r b^{\prime}(\alpha+r)-b(\alpha+r)+b(\alpha) \\
& =\int_{0}^{r} \int_{t}^{r} b^{\prime \prime}(\alpha+s) d s d t,
\end{aligned}
$$

so that

$$
h_{\alpha}^{\prime}(r)=r b_{\alpha}^{\prime \prime}(r)=r b^{\prime \prime}(\alpha+r) .
$$

It follows from (4-6) that $h_{\alpha}(0)=0$, and since $b^{\prime \prime}(x) \geq 0$, it follows from (4-7) that $h_{\alpha}(x)$ is increasing for $x>0$ and decreasing for $x<0$. Note that $h_{\alpha}(-\alpha)=b(\alpha)$ and $b_{\alpha}(-\alpha)=h(\alpha)$.

If we let $\tilde{b}(r)=b(-r)$ and $\tilde{h}(r)=h(-r)$, then

$$
\begin{array}{lll}
b_{\alpha}(-r)=\tilde{b}_{-\alpha}(r), & b_{\alpha}^{\prime}(-r)=-\tilde{b}_{-\alpha}^{\prime}(r), & b_{\alpha}^{\prime \prime}(-r)=\tilde{b}_{-\alpha}^{\prime \prime}(r) ; \\
h_{\alpha}(-r)=\tilde{h}_{-\alpha}(r), & h_{\alpha}^{\prime}(-r)=-\tilde{h}_{-\alpha}^{\prime}(r), & h_{\alpha}^{\prime \prime}(-r)=\tilde{h}_{-\alpha}^{\prime \prime}(r) .
\end{array}
$$

These identities often allow us to verify identities by considering only $r \geq 0$. Also, if $b$ is an even function, so is $h$, and the identities in (4-8) hold for $\tilde{b}=b$ and $\tilde{h}=h$.

\subsection{Elementary estimates for $b_{\alpha}$ and $h_{\alpha}$.}

Proposition 4.3. Suppose that $b$ satisfies (3-1)-(3-5). Let $\alpha, r \in \mathbb{R}$, and let $\epsilon>0$.

(a) $b_{\alpha}((1+\epsilon) r) \geq(1+\epsilon) b_{\alpha}(r)$.

(b) $0 \leq b_{\alpha}(r) \leq r b_{\alpha}^{\prime}(r) \leq \epsilon^{-1} b_{\alpha}((1+\epsilon) r)$ and in particular $r b_{\alpha}^{\prime}(r) \leq b_{\alpha}(2 r)$.

(c) $0 \leq h_{\alpha}(r) \leq \epsilon^{-1} b_{\alpha}((1+\epsilon) r)-b_{\alpha}(r)$, and in particular $h_{\alpha}(r) \leq b_{\alpha}(2 r)-b_{\alpha}(r)$.

(d) the function $r \mapsto r^{-1} b_{\alpha}(r)$ is monotone increasing.

Proof. Suppose $r \geq 0$. Since $b_{\alpha}^{\prime}$ is monotone increasing and $b_{\alpha}(0)=0$, we have

$$
\begin{aligned}
b_{\alpha}((1+\epsilon) r) & \geq b_{\alpha}(r)+\int_{r}^{(1+\epsilon) r} b_{\alpha}^{\prime}(s) d s \geq b_{\alpha}(r)+\epsilon r b_{\alpha}^{\prime}(r) \\
& \geq b_{\alpha}(r)+\epsilon \int_{0}^{r} b_{\alpha}^{\prime}(s) d s=(1+\epsilon) b_{\alpha}(r) .
\end{aligned}
$$

This proves (a) and (b) for $r \geq 0$; the case $r \leq 0$ then follows by applying these inequalities to $\tilde{b}$. Since $h_{\alpha}(r)=r b_{\alpha}^{\prime}(r)-b_{\alpha}(r)$, part (c) follows from (b). Finally,

$$
\left(\frac{b_{\alpha}(r)}{r}\right)^{\prime}=\frac{r b_{\alpha}^{\prime}(r)-b_{\alpha}(r)}{r^{2}}=\frac{h_{\alpha}(r)}{r^{2}} \geq 0,
$$

which gives (d).

The next proposition describes the relationship between the sizes of the functions $b_{\alpha}$ and $h_{\alpha}$. 
Proposition 4.4. Suppose that b satisfies (3-1)-(3-5). Then

$$
\begin{array}{ll}
\alpha \geq 0 \text { implies } & \begin{cases}b_{\alpha}(r) \leq h_{\alpha}(r) & \text { if } r \notin[-2 \alpha, 0] ; \\
b_{\alpha}(r) \geq h_{\alpha}(r) & \text { if } r \in[-2 \alpha, 0] .\end{cases} \\
\alpha \leq 0 \quad \text { implies } & \begin{cases}b_{\alpha}(r) \leq h_{\alpha}(r) & \text { if } r \notin[0,-2 \alpha] ; \\
b_{\alpha}(r) \geq h_{\alpha}(r) & \text { if } r \in[0,-2 \alpha]\end{cases}
\end{array}
$$

Proof. The case $\alpha \leq 0$ follows from the results for $\alpha \geq 0$ by using the identities in (4-8), so we can assume that $\alpha \geq 0$. Let $\varphi(r)=r b_{\alpha}^{\prime}(r)$ and $\psi(r)=r b_{\alpha}^{\prime \prime}(r)$. Note that $\varphi(0)=\psi(0)=0$. For $r \neq 0$ we can use Cauchy's mean value theorem to establish the existence of points $s$ and $t$ with $0<|t|<|s|<|r|$ such that

$$
\begin{aligned}
\frac{r b_{\alpha}^{\prime}(r)}{b_{\alpha}(r)} & =\frac{\varphi(r)}{b_{\alpha}(r)}=\frac{\varphi(r)-\varphi(0)}{b_{\alpha}(r)-b_{\alpha}(0)}=\frac{\varphi^{\prime}(s)}{b_{\alpha}^{\prime}(s)}=\frac{s b_{\alpha}^{\prime \prime}(s)+b_{\alpha}^{\prime}(s)}{b_{\alpha}^{\prime}(s)}=1+\frac{\psi(s)}{b_{\alpha}^{\prime}(s)} \\
& =1+\frac{\psi(s)-\psi(0)}{b_{\alpha}^{\prime}(s)-b_{\alpha}^{\prime}(0)}=1+\frac{\psi^{\prime}(t)}{b_{\alpha}^{\prime \prime}(t)}=1+\frac{b_{\alpha}^{\prime \prime}(t)+t b_{\alpha}^{\prime \prime \prime}(t)}{b_{\alpha}^{\prime \prime}(t)}=2+\frac{t b_{\alpha}^{\prime \prime \prime}(t)}{b_{\alpha}^{\prime \prime}(t)} \\
& =2+\frac{t b^{\prime \prime \prime}(\alpha+t)}{b^{\prime \prime}(\alpha+t)} .
\end{aligned}
$$

If $r>0$ it follows that $t>0$, in which case the last fraction is nonnegative, so $r b_{\alpha}^{\prime}(r) \geq 2 b_{\alpha}(r)$, and hence $b_{\alpha}(r) \leq h_{\alpha}(r)$. Also, if $-\alpha \leq r<0$ it follows that $-\alpha<t<0$, in which case the last fraction is nonpositive, so $r b_{\alpha}^{\prime}(r) \leq 2 b_{\alpha}(r)$, and hence $b_{\alpha}(r) \geq h_{\alpha}(r)$.

Next, observe that since $b$ is an even function, we have

$$
\begin{gathered}
h_{\alpha}(-2 \alpha)-b_{\alpha}(-2 \alpha)=2 \alpha b^{\prime}(\alpha)-2 \alpha b^{\prime}(\alpha)=0, \\
h_{\alpha}^{\prime}(-2 \alpha)-b_{\alpha}^{\prime}(-2 \alpha)=2\left(b^{\prime}(\alpha)-\alpha b^{\prime \prime}(\alpha)\right) \leq 0,
\end{gathered}
$$

and $h_{\alpha}^{\prime \prime}(r)-b_{\alpha}^{\prime \prime}(r)=r b^{\prime \prime \prime}(\alpha+r) \geq 0$ for $r \leq-\alpha$. It follows that $\left(h_{\alpha}-b_{\alpha}\right)(r) \geq 0$ for $r \leq-2 \alpha$ and $\left(h_{\alpha}-b_{\alpha}\right)(r) \leq 0$ for $-2 \alpha \leq r \leq-\alpha$.

If $b$ satisfies (3-1), then $b$ and $h$ are monotone increasing on the positive axis and map $[0, \infty)$ to itself, so we can define inverse functions $b^{-1}, h^{-1}:[0, \infty) \rightarrow[0, \infty)$. Then both $b^{-1}$ and $h^{-1}$ are monotone increasing. Although the functions $b$ and $h$ are in general not comparable and do not satisfy a doubling property of the form $f(2 x) \leq C f(x)$, we have the following result about the inverse functions.

Proposition 4.5. Suppose $b$ satisfies (3-1)-(3-5). Then for any $\lambda \geq 0$,

$$
\begin{aligned}
h^{-1}(\lambda) & \leq b^{-1}(\lambda) \leq 2 h^{-1}(\lambda), \\
b^{-1}(2 \lambda) & \leq 2 b^{-1}(\lambda) \leq 2 b^{-1}(2 \lambda), \\
h^{-1}(2 \lambda) & \leq 2 h^{-1}(\lambda) \leq 2 h^{-1}(2 \lambda) .
\end{aligned}
$$


Proof. Let $\lambda \geq 0$, and let $r=b^{-1}(\lambda) \geq 0$. It follows from Proposition 4.4 that $\lambda=b(r) \leq h(r)$, and since $h^{-1}$ is monotone increasing, $h^{-1}(\lambda) \leq r=b^{-1}(\lambda)$. This gives the first inequality. Next, if $\lambda \geq 0$ and if $r \geq 0$ satisfies $\lambda=h(r / 2)$, it follows from Proposition 4.3(c) that $\lambda=h(r / 2) \leq b(r)-b(r / 2) \leq b(r)$. Thus $b^{-1}(\lambda) \leq r=2 h^{-1}(\lambda)$. Finally we have

$b(2 r)=b(r)+\int_{r}^{2 r} b^{\prime}(t) d t=b(r)+\int_{0}^{r} b^{\prime}(t+r) d t \geq b(r)+\int_{0}^{r} b^{\prime}(t) d t=2 b(r)$, and this gives $b^{-1}(2 \lambda) \leq 2 b^{-1}(\lambda)$. The same argument works for any function with monotone increasing derivative, and in particular for $h$.

In general, the functions $\left\{b_{\alpha}\right\}$ are not even for $\alpha \neq 0$, even if $b$ is even. ${ }^{7}$ The next proposition clarifies this lack of symmetry.

Proposition 4.6. Suppose that $b$ satisfies (3-1)-(3-5). Let $r>0$. Then $\alpha \geq 0$ implies $\left\{\begin{array}{l}b_{\alpha}(-r) \leq b_{\alpha}(r), \\ h_{\alpha}(-r) \leq h_{\alpha}(r) ;\end{array} \quad\right.$ and $\quad \alpha \leq 0$ implies $\left\{\begin{array}{l}b_{\alpha}(-r) \geq b_{\alpha}(r), \\ h_{\alpha}(-r) \geq h_{\alpha}(r) .\end{array}\right.$

Proof. Suppose first that $\alpha \geq 0$. Since $b_{\alpha}(0)=b_{\alpha}^{\prime}(0)=0$, for $r>0$ we have

$$
b_{\alpha}(+r)=\int_{0}^{r} b^{\prime \prime}(\alpha+t)(r-t) d t \quad \text { and } \quad b_{\alpha}(-r)=\int_{0}^{r} b^{\prime \prime}(\alpha-t)(r-t) d t .
$$

Thus $b_{\alpha}(+r)-b_{\alpha}(-r)=\int_{0}^{r}(r-t) \int_{\alpha-t}^{\alpha+t} b^{\prime \prime \prime}(s) d s d t$. If $\alpha-t \geq 0$, then the inner integral is positive, so $b_{\alpha}(+r)-b_{\alpha}(-r) \geq 0$. On the other hand, if $\alpha-t<0$ we have

$$
\int_{\alpha-t}^{\alpha+t} b^{\prime \prime \prime}(s) d s=\int_{\alpha-t}^{t-\alpha} b^{\prime \prime \prime}(s) d s+\int_{t-\alpha}^{t+\alpha} b^{\prime \prime \prime}(s) d s=\int_{t-\alpha}^{t+\alpha} b^{\prime \prime \prime}(s) d s \geq 0
$$

since $b^{\prime \prime \prime}(-s)=-b^{\prime \prime \prime}(s)$, and so $\int_{\alpha-t}^{t-\alpha} b^{\prime \prime \prime}(s) d s=0$. Thus $b_{\alpha}(+r)-b_{\alpha}(-r) \geq 0$ again, which is the first inequality if $\alpha>0$.

Next, for $r>0$ we have

$$
\begin{aligned}
h_{\alpha}(+r) & =\int_{0}^{r} h_{\alpha}^{\prime}(s) d s=\int_{0}^{r} s b_{\alpha}^{\prime \prime}(s) d s, \\
-h_{\alpha}(-r) & =\int_{-r}^{0} h_{\alpha}^{\prime}(s) d s=\int_{0}^{r}(-s) b_{\alpha}^{\prime \prime}(-s) d s,
\end{aligned}
$$

so $h_{\alpha}(r)-h_{\alpha}(-r)=\int_{0}^{r} s\left(b_{\alpha}^{\prime \prime}(s)-b_{\alpha}^{\prime \prime}(-s)\right) d s=\int_{0}^{r} s\left(b^{\prime \prime}(\alpha+s)-b^{\prime \prime}(\alpha-s)\right) d s$. But for $\alpha, s \geq 0$ we have $b^{\prime \prime}(\alpha+s)-b^{\prime \prime}(\alpha-s) \geq 0$, and so $h_{\alpha}(r)-h_{\alpha}(-r) \geq 0$, completing the proof for $\alpha>0$. The case $\alpha<0$ follows similarly, using (4-8).

${ }^{7}$ However, if $b(r)=r^{2}$, then $b_{\alpha}(r)=r^{2}$ for all $\alpha \in \mathbb{R}$. 
4.7. Estimates for $\left|\left\{r \in \mathbb{R} \mid \boldsymbol{b}_{\alpha}(\boldsymbol{r})<\boldsymbol{\tau}^{-1}\right\}\right|$. We need estimates for the measure of the intervals on which the functions $b_{\alpha}$ are bounded by a constant $\tau^{-1}$. In case $\alpha \geq 0$, these depend on estimates of the functions $b_{\alpha}$ and $h_{\alpha}$ on the interval $[-\alpha, 0]$. The key fact is that there is an interval $[\alpha-\eta(\alpha), \alpha]$ on which $b(r), b^{\prime}(r)$, and $b^{\prime \prime}(r)$ are essentially constant.

Set $\eta(\alpha)=\left(b(\alpha) / b^{\prime}(\alpha)\right) /(2 C)$ where $C>1$ is the constant in (3-4). Note that $\eta(\alpha)<\alpha / 2$. It follows from (3-4) that for $j \in\{0,1,2\}$,

$$
\frac{1}{2 C^{2}} b^{(j)}(\alpha) \leq \eta(\alpha) b^{(j+1)}(\alpha) \leq \frac{1}{2} b^{(j)}(\alpha) .
$$

Proposition 4.8. Suppose that $b$ satisfies (3-1)-(3-5), and let $\alpha>0$.

(a) The functions $\left\{b^{(j)}\right\}$ satisfy

(i) $\frac{1}{2} b^{(j)}(\alpha) \leq b^{(j)}(r) \leq b^{(j)}(\alpha)$ if $j \in\{0,1,2\}$ and $\alpha-\eta(\alpha) \leq r \leq \alpha$;

(ii) $0 \leq b^{(j)}(r) \leq\left(1-\left(4 C^{2}\right)^{-1}\right) b^{(j)}(\alpha)$ if $j \in\{0,1\}$ and $0 \leq r \leq \alpha-\eta(\alpha)$.

(b) The function $h_{\alpha}$ satisfies

(i) $\frac{1}{4} r^{2} b^{\prime \prime}(\alpha) \leq h_{\alpha}(-r) \leq \frac{1}{2} r^{2} b^{\prime \prime}(\alpha)$ if $0 \leq r \leq \eta(\alpha)$;

(ii) $r b^{\prime}(\alpha) /\left(16 C^{2}\right) \leq b_{\alpha}(-r) \leq r b^{\prime}(\alpha)$ if $\eta(\alpha) \leq r \leq 2 \alpha$;

(iii) $b(\alpha)+h(r-\alpha) \leq h_{\alpha}(-r) \leq 4 h(r)$ if $\alpha \leq r$.

(c) The function $b_{\alpha}$ satisfies

(a) $\frac{1}{4} r^{2} b^{\prime \prime}(\alpha) \leq b_{\alpha}(-r) \leq \frac{1}{2} r^{2} b^{\prime \prime}(\alpha)$ if $0 \leq r \leq \eta(\alpha)$;

(b) $r b^{\prime}(\alpha) /\left(16 C^{2}\right) \leq b_{\alpha}(-r) \leq r b^{\prime}(\alpha)$ if $\eta(\alpha) \leq r \leq 2 \alpha$;

(c) $b(r / 2) \leq b_{\alpha}(-r) \leq 3 b(r)$ if $r \geq 2 \alpha$.

Proof. (a) Since the functions $b^{(j)}(r)$ are monotone increasing for $j \leq 3$ and $r>0$, it follows from (4-9) that for $\alpha-\eta(\alpha) \leq r \leq \alpha$ we have

$$
b^{(j)}(\alpha)-b^{(j)}(r) \leq(\alpha-r) b^{(j+1)}(\alpha) \leq \eta(\alpha) b^{(j+1)}(\alpha) \leq \frac{1}{2} b^{(j)}(\alpha),
$$

and so $b^{(j)}(\alpha) / 2 \leq b^{(j)}(r) \leq b^{(j)}(\alpha)$. This gives the first set of inequalities. Next, if $0 \leq r \leq \alpha-\eta(\alpha)$ and $j \in\{0,1\}$, it follows from the first inequality that $b^{(j+1)}(\alpha-\eta(\alpha)) \geq b^{(j+1)}(\alpha) / 2$, and so we have

$$
b^{(j)}(\alpha)-b^{(j)}(r) \geq \int_{\alpha-\eta(\alpha)}^{\alpha} b^{(j+1)}(t) d t \geq \frac{1}{2} \eta(\alpha) b^{(j+1)}(\alpha) \geq b^{(j)}(\alpha) /\left(4 C^{2}\right) .
$$

Thus $b^{(j)}(r) \leq\left(1-\left(4 C^{2}\right)^{-1}\right) b^{(j)}(\alpha)$. This proves (a).

(b) Using Equation (4-6) and the monotonicity of $b^{\prime \prime}$,we have

$$
h_{\alpha}(-r)=\int_{\alpha-r}^{\alpha} \int_{\alpha-r}^{t} b^{\prime \prime}(s) d s d t \leq \frac{1}{2} r^{2} b^{\prime \prime}(\alpha)
$$


for $0 \leq r \leq \alpha$. On the other hand, if $r \leq \eta(\alpha)$, we have

$$
h_{\alpha}(-r)=\int_{\alpha-r}^{\alpha} \int_{\alpha-r}^{t} b^{\prime \prime}(s) d s d t \geq \frac{1}{2} r^{2} b^{\prime \prime}(\alpha-r) \geq \frac{1}{2} r^{2} b^{\prime \prime}(\alpha-\eta(\alpha)) \geq \frac{1}{4} r^{2} b^{\prime \prime}(\alpha)
$$

by the first inequality in part (a). This gives the first set of inequalities. Next, recall from (4-7) that $h_{\alpha}^{\prime}(-r)=-r b^{\prime \prime}(\alpha-r)$, and so $h_{\alpha}^{\prime}(-r)$ is monotone decreasing for $r \geq 0$. Using the first inequality in (b) and (4-9), it follows that $h_{\alpha}(-\eta(\alpha)) \geq 4^{-1} \eta(\alpha)^{2} b^{\prime \prime}(\alpha) \geq\left(16 C^{4}\right)^{-1} b(\alpha)$ and $h_{\alpha}(-\alpha)=b(\alpha)$. Thus we have $\left(16 C^{4}\right)^{-1} b(\alpha) \leq h_{\alpha}(-r) \leq b(\alpha)$. This gives the second set of inequalities. To prove the third set of inequalities, note that

$$
\begin{aligned}
h_{\alpha}(-r) & -h(r-\alpha) \\
& =\left(-r b^{\prime}(\alpha-r)-b(\alpha-r)+b(\alpha)\right)-\left((r-\alpha) b^{\prime}(r-\alpha)-b(r-\alpha)\right) \\
& =\alpha b^{\prime}(r-\alpha)+b(\alpha) .
\end{aligned}
$$

If $r \geq \alpha$, then $\alpha b^{\prime}(r-\alpha) \geq 0$, so $h_{\alpha}(-r) \geq h(r-\alpha)+b(\alpha)$. Also, if $r \geq \alpha$, $\alpha b^{\prime}(r-\alpha) \leq r b^{\prime}(r)=h(r)+b(r) \leq 2 h(r)$. Thus

$$
h_{\alpha}(-r) \leq h(r-\alpha)+b(\alpha)+2 h(r) \leq 4 h(r) .
$$

This proves (b).

(c) First suppose $0 \leq r \leq \eta(\alpha)$. Then $b^{\prime \prime}(\alpha-r) \geq b^{\prime \prime}(\alpha-\eta(\alpha)) \geq b^{\prime \prime}(\alpha) / 2$ by the first inequality in (a). But $b_{\alpha}(-r)=\int_{\alpha-r}^{\alpha} \int_{t}^{\alpha} b^{\prime \prime}(s) d s d t$, so using the monotonicity of $b^{\prime \prime}$ it follows that

$$
\frac{1}{4} r^{2} b^{\prime \prime}(\alpha) \leq \frac{1}{2} r^{2} b^{\prime \prime}(\alpha-r) \leq b_{\alpha}(-r) \leq \frac{1}{2} r^{2} b^{\prime \prime}(\alpha) .
$$

This is the first inequality. Next suppose $\eta(\alpha) \leq r \leq 2 \alpha$. We have $b(\alpha-r) \leq b(\alpha)$, and hence

$$
b_{\alpha}(-r)=r b^{\prime}(\alpha)-(b(\alpha)-b(\alpha-r)) \leq r b^{\prime}(\alpha) .
$$

On the other hand, using the second estimate in (a) for $b^{\prime}$ and the monotonicity of $b^{\prime}$, we have

$$
\begin{aligned}
b(\alpha)-b(\alpha-r) & =\int_{\alpha-r}^{\alpha-\eta(\alpha)} b^{\prime}(s) d s+\int_{\alpha-\eta(\alpha)}^{\alpha} b^{\prime}(s) d s \\
& \leq\left(1-\left(4 C^{2}\right)^{-1}\right) b^{\prime}(\alpha)(r-\eta(\alpha))+\eta(\alpha) b^{\prime}(\alpha) \\
& =\left(1-\left(4 C^{2}\right)^{-1}\right) r b^{\prime}(\alpha)+\left(4 C^{2}\right)^{-1} \eta(\alpha) b^{\prime}(\alpha) .
\end{aligned}
$$

Thus if $2 \eta(\alpha) \leq r$, it follows that $b(\alpha)-b(\alpha-r) \leq\left(1-\left(8 C^{2}\right)^{-1}\right) r b^{\prime}(\alpha)$, and hence $b_{\alpha}(-r)=r b^{\prime}(\alpha)-(b(\alpha)-b(\alpha-r)) \geq\left(8 C^{2}\right)^{-1} r b^{\prime}(\alpha)$. It remains to establish a lower bound for $b_{\alpha}(-r)$ for $\eta(\alpha) \leq r \leq 2 \eta(\alpha)$. In this case, using the fact that 
$\eta(\alpha) \leq \alpha / 2$ so that $\alpha-\eta(\alpha)>0$, we have

$$
\begin{aligned}
b_{\alpha}(-r) & \geq b_{\alpha}(-\eta(\alpha))=\int_{\alpha-\eta(\alpha)}^{\alpha} \int_{t}^{\alpha} b^{\prime \prime}(s) d s d t \geq \frac{1}{2} \eta(\alpha)^{2} b^{\prime \prime}(\alpha-\eta(\alpha)) \\
& \geq \frac{1}{4} \eta(\alpha)^{2} b^{\prime \prime}(\alpha) \geq\left(8 C^{2}\right)^{-1} \eta(\alpha) b^{\prime}(\alpha) \geq\left(16 C^{2}\right)^{-1} r b^{\prime}(\alpha) .
\end{aligned}
$$

This gives the second inequality. Finally if $r \geq 2 \alpha$,

$$
b_{\alpha}(-r)=b(r-\alpha)-b(\alpha)+r b^{\prime}(\alpha) \geq b(r / 2)+\alpha b^{\prime}(\alpha)-b(\alpha) \geq b(r / 2),
$$

and by Proposition 4.3, $b_{\alpha}(-r) \leq b(r)+r b^{\prime}(\alpha) \leq b(r)+r b^{\prime}(r / 2) \leq 3 b(r)$.

We can now derive estimates for the measure of the set $\left|\left\{r \in \mathbb{R} \mid b_{\alpha}(r) \leq \tau^{-1}\right\}\right|$.

Proposition 4.9. There are constants $c<1<C$ such that

$$
\begin{aligned}
& \text { if } 0<\tau^{-1} \leq b(\alpha) \text {, then } \\
& \quad c\left(\tau b^{\prime \prime}(\alpha)\right)^{-1 / 2} \leq\left|\left\{r \in \mathbb{R} \mid b_{\alpha}(r) \leq \tau^{-1}\right\}\right| \leq C\left(\tau b^{\prime \prime}(\alpha)\right)^{-1 / 2} ;
\end{aligned}
$$

(4-11) $\quad$ if $b(\alpha) \leq \tau^{-1} \leq h(\alpha)$, then

$$
c\left(\tau b^{\prime}(\alpha)\right)^{-1} \leq\left|\left\{r \in \mathbb{R} \mid b_{\alpha}(r) \leq \tau^{-1}\right\}\right| \leq C\left(\tau b^{\prime}(\alpha)\right)^{-1} ;
$$

if $h(\alpha) \leq \tau^{-1}$ then

$$
c b^{-1}\left(\tau^{-1}\right) \leq\left|\left\{r \in \mathbb{R} \mid b_{\alpha}(r) \leq \tau^{-1}\right\}\right| \leq C b^{-1}\left(\tau^{-1}\right) .
$$

In particular, for any $\tau>0$,

$$
\begin{aligned}
c\left(\tau^{1 / 2} b^{\prime \prime}(\alpha)^{1 / 2}\right. & \left.+\tau b^{\prime}(\alpha)+\left(b^{-1}\left(\tau^{-1}\right)\right)^{-1}\right)^{-1} \\
& \leq\left|\left\{r \in \mathbb{R} \mid b_{\alpha}(r) \leq \tau^{-1}\right\}\right| \\
& \leq C\left(\tau^{-1 / 2} b^{\prime \prime}(\alpha)^{-1 / 2}+\tau^{-1} b^{\prime}(\alpha)^{-1}+b^{-1}\left(\tau^{-1}\right)\right) .
\end{aligned}
$$

Proof. Since $b_{\alpha}(r)=b_{-\alpha}(-r)$, it suffices to establish the estimates for $\alpha>0$. Now $b_{\alpha}(r)=\tau^{-1}$ has two roots, $-r_{1}<0<r_{2}$, and so $\left|\left\{r \in \mathbb{R} \mid b_{\alpha}(r) \leq \tau^{-1}\right\}\right|=r_{1}+r_{2}$. By Proposition 4.6, $r_{2} \leq r_{1}$, and so $r_{1} \leq\left|\left\{r \in \mathbb{R} \mid b_{\alpha}(r) \leq \tau^{-1}\right\}\right| \leq 2 r_{1}$. Thus in proving (4-10), (4-11), and (4-12), it suffices to obtain estimates for $r_{1}$.

Since $b_{\alpha}\left(-r_{1}\right)=\tau^{-1}$, it follows from Proposition 4.8(c) that

$$
\begin{array}{rcrrrl}
(4-14) & r_{1} & \leq \eta(\alpha) & \text { implies } & \sqrt{2}\left(\tau b^{\prime \prime}(\alpha)\right)^{-1 / 2} \leq r_{1} \leq 2\left(\tau b^{\prime \prime}(\alpha)\right)^{-1 / 2} ; \\
(4-15) & \eta(\alpha) \leq r_{1} \leq 2 \alpha & \text { implies } & \left(\tau b^{\prime}(\alpha)\right)^{-1} \leq r_{1} \leq 16 C^{2}\left(\tau b^{\prime}(\alpha)\right)^{-1} ; \\
(4-16) & r_{1} & \geq 2 \alpha & \text { implies } & b^{-1}\left(3^{-1} \tau^{-1}\right) & \leq r_{1} \leq 2 b^{-1}\left(\tau^{-1}\right) .
\end{array}
$$$$
\text { (4-16) } \quad r_{1} \geq 2 \alpha
$$

It follows from (4-9) and the first inequality in part (c) of Proposition 4.8 that $\left(16 C^{4}\right)^{-1} b(\alpha) \leq b_{\alpha}(-\eta(\alpha)) \leq 8^{-1} b(\alpha)$. Remembering that $\tau^{-1}=b_{\alpha}\left(-r_{1}\right)$ and that $b_{\alpha}(r)$ is decreasing for $r<0$, it follows that if $\tau^{-1} \leq\left(16 C^{4}\right)^{-1} b(\alpha)$, then 
$b_{\alpha}\left(-r_{1}\right)=\tau^{-1} \leq\left(16 C^{4}\right)^{-1} b(\alpha) \leq b_{\alpha}(-\eta(\alpha))$, and so

$$
\tau^{-1} \leq b(\alpha) /\left(16 C^{4}\right) \quad \text { implies } \quad r_{1} \leq \eta(\alpha) .
$$

Similarly, if $\tau^{-1} \geq 8^{-1} b(\alpha)$, then $b_{\alpha}\left(-r_{1}\right)=\tau^{-1} \geq 8^{-1} b(\alpha) \geq b_{\alpha}(-\eta(\alpha))$, and so

$$
\tau^{-1} \geq b(\alpha) / 8 \quad \text { implies } \quad r_{1} \geq \eta(\alpha) .
$$

Now $b_{\alpha}(-2 \alpha)=b(-\alpha)-b(\alpha)+2 \alpha b^{\prime}(\alpha)=2 h(\alpha)+2 b(\alpha)$. Since $0 \leq b(\alpha) \leq h(\alpha)$ by Proposition 4.4, it follows that $2 h(\alpha) \leq b_{\alpha}(-2 \alpha) \leq 4 h(\alpha)$. If $\tau^{-1} \leq 2 h(\alpha)$, then $b_{\alpha}\left(-r_{1}\right)=\tau^{-1} \leq 2 h(\alpha) \leq b_{\alpha}(-2 \alpha)$, and so

$$
\tau^{-1} \leq 2 h(\alpha) \text { implies } \quad r_{1} \leq 2 \alpha,
$$

while if $\tau^{-1} \geq 4 h(\alpha)$, then $b_{\alpha}\left(-r_{1}\right)=\tau^{-1} \geq b_{\alpha}(-2 \alpha)$, and so

$$
\tau^{-1} \geq 4 h(\alpha) \text { implies } \quad r_{1} \geq 2 \alpha .
$$

The implications in (4-17) and (4-14) establish (4-10) if $\tau^{-1} \leq\left(16 C^{4}\right)^{-1} b(\alpha)$, those in (4-17), (4-18) and (4-15) establish (4-11) if $b(\alpha) / 8 \leq \tau^{-1} \leq 2 h(\alpha)$, and those in (4-20) and (4-16), together with Proposition 4.3(a), establish (4-12) if $\tau^{-1} \geq 4 h(\alpha)$.

Thus to complete the proof, it suffices to show that, given constants $A \leq 1 \leq B$, there is a constant $C$ such that

$$
\begin{array}{lll}
A b(\alpha) \leq \tau^{-1} \leq B b(\alpha) & \text { implies } & C^{-1} \leq \tau b^{\prime}(\alpha) /\left(\tau b^{\prime \prime}(\alpha)\right)^{1 / 2} \leq C \\
A h(\alpha) \leq \tau^{-1} \leq B h(\alpha) & \text { implies } & C^{-1} \leq \tau b^{\prime}(\alpha) b^{-1}\left(\tau^{-1}\right) \leq C .
\end{array}
$$

To check (4-21), observe that $\tau b^{\prime}(\alpha) / \sqrt{\tau b^{\prime \prime}(\alpha)}=\sqrt{\tau b(\alpha)} \sqrt{b^{\prime}(\alpha)^{2} /\left(b(\alpha) b^{\prime \prime}(\alpha)\right)}$. It follows from the hypothesis (3-4) that this last quantity is bounded above and below by constants independent of $\alpha$.

To check (4-22), observe that $\tau b^{\prime}(\alpha)=(\tau / \alpha)(h(\alpha)+b(\alpha))$, and so the hypothesis of (4-22) gives

$$
\frac{1}{B \alpha} \leq \frac{\tau}{\alpha} h(\alpha) \leq \tau b^{\prime}(\alpha)=\frac{\tau}{\alpha} h(\alpha)+\frac{\tau}{\alpha} b(\alpha) \leq \frac{1}{A \alpha}+\frac{\tau}{\alpha} b(\alpha) .
$$

But $A h(\alpha) \leq \tau^{-1}$ and Proposition 4.5 imply $\alpha \leq h^{-1}\left((A \tau)^{-1}\right) \leq b^{-1}\left((A \tau)^{-1}\right)$, and so $b(\alpha) \leq(A \tau)^{-1}$. Thus the hypothesis of (4-22) implies

$$
\frac{1}{B} \frac{b^{-1}\left(\tau^{-1}\right)}{\alpha} \leq \tau b^{\prime}(\alpha) b^{-1}\left(\tau^{-1}\right) \leq \frac{2}{A} \frac{b^{-1}\left(\tau^{-1}\right)}{\alpha} .
$$

Also, the hypothesis can be written $h^{-1}\left((B \tau)^{-1}\right) \leq \alpha \leq h^{-1}\left((A \tau)^{-1}\right)$, and so again by Proposition 4.5, it follows that $b^{-1}\left((B \tau)^{-1}\right) \leq 2 \alpha$ and $\alpha \leq b^{-1}\left((A \tau)^{-1}\right)$. 
Choose $m, n>0$ so that $2^{-m-1}<A \leq 2^{-m}$ and $2^{n-1} \leq B \leq 2^{n}$. Then

$$
b^{-1}\left(\frac{1}{\tau}\right)=b^{-1}\left(\frac{2^{n}}{2^{n} \tau}\right) \leq 2^{n} b^{-1}\left(\frac{1}{B \tau}\right) \leq 2^{n+1} \alpha
$$

so $b^{-1}\left(\tau^{-1}\right) \leq 4 B \alpha$. Also

$$
2 A^{-1} b^{-1}\left(\frac{1}{\tau}\right) \geq 2^{m+1} b^{-1}\left(\frac{1}{\tau}\right) \geq b^{-1}\left(\frac{2^{m+1}}{\tau}\right) \geq b^{-1}\left(\frac{1}{A \tau}\right) \geq \alpha
$$

so $b^{-1}\left(\tau^{-1}\right) \geq A \alpha / 2$. Combining these estimates with (4-23), it follows that $A(2 B)^{-1} \leq \tau b^{\prime}(\alpha) b^{-1}\left(\tau^{-1}\right) \leq 8 A^{-1} B$, which completes the proof.

4.10. Estimates of integrals. In our analysis, a key role is played by the functions

$$
\begin{aligned}
\varphi(\alpha, \tau) & =\int_{-\infty}^{+\infty} e^{-2 \tau b_{\alpha}(r)} d r, \quad \text { and } \\
N_{\alpha}(\eta, \tau) & =\int_{-\infty}^{+\infty} e^{2\left(\eta r-\tau b_{\alpha}(r)\right)} d r,
\end{aligned}
$$

as well as the quantities

$$
\begin{aligned}
& \mu(\alpha, \tau)=\left|\left\{r \in \mathbb{R} \mid b_{\alpha}(r) \leq \tau^{-1}\right\}\right|, \quad \text { and } \\
& \nu(\alpha, \tau)=\left|\left\{r \in \mathbb{R} \mid h_{\alpha}(r) \leq \tau^{-1}\right\}\right|
\end{aligned}
$$

We will frequently make the substitution $\eta=\tau b_{\alpha}^{\prime}(\lambda)$ and use the following identity.

Proposition 4.11. $N_{\alpha}\left(\tau b_{\alpha}^{\prime}(\lambda), \tau\right)=e^{2 \tau h_{\alpha}(\lambda)} \int_{-\infty}^{+\infty} e^{-2 \tau b_{\alpha+\lambda}(r)} d r$.

Proof. We have $N_{\alpha}\left(\tau b_{\alpha}^{\prime}(\lambda), \tau\right)=\int_{-\infty}^{+\infty} e^{2 \tau\left(b_{\alpha}^{\prime}(\lambda) r-b_{\alpha}(r)\right)} d r$. Making the change of variables $r \rightarrow r+\lambda$ and then using identity (4-4) gives the stated formula.

The following simple estimates, in particular, relate $\varphi(\alpha, \tau)$ and $\mu(\alpha, \tau)$.

Proposition 4.12. For $j, k \geq 0$ there are constants $A_{j, k}$ (independent of $b$ ) such that if $b$ satisfies (3-1) and $\mu=|\{r \in \mathbb{R} \mid b(r) \leq 1\}|$, then

$$
\int_{-\infty}^{+\infty} b(r)^{j}|r|^{k} e^{-b(r)} d r \leq A_{j, k} \mu^{k+1} \leq A_{j, k} e^{k+1}\left(\int_{-\infty}^{+\infty} e^{-b(r)} d r\right)^{k+1} .
$$

Proof. Let $[\alpha, \beta]$ be the interval on which $b(r) \leq 1$. Then

$$
\int_{-\infty}^{+\infty} e^{-b(r)} d r \geq \int_{\alpha}^{\beta} e^{-b(r)} d r \geq e^{-1}(\beta-\alpha)=e^{-1} \mu .
$$

This establishes the right side of the inequality in Proposition 4.12. To establish the left hand side, it suffices to make estimates on the positive real axis. Let $r_{m} \geq 0$ 
be the point such that $b\left(r_{m}\right)=m$. Then $r_{0}=0$ and $r_{1}=|\{r \geq 0 \mid b(r) \leq 1\}| \leq \mu$. We have

$$
\int_{0}^{+\infty} b(r)^{j} r^{k} e^{-b(r)} d r=\sum_{m=1}^{\infty} \int_{r_{m-1}}^{r_{m}} b(r)^{j} r^{k} e^{-b(r)} d r \leq \frac{e}{k+1} \sum_{m=1}^{\infty} r_{m}^{k+1} m^{j} e^{-m} .
$$

On the other hand, since $b^{\prime}$ is monotone increasing and $1=b\left(r_{1}\right) \leq r_{1} b^{\prime}\left(r_{1}\right)$, we have

$$
m=b\left(r_{m}\right)=1+\int_{r_{1}}^{r_{m}} b^{\prime}(s) d s \geq 1+b^{\prime}\left(r_{1}\right)\left(r_{m}-r_{1}\right) \geq 1+\left(\left(r_{m}-r_{1}\right)\right) / r_{1} .
$$

Thus $r_{m} \leq m r_{1} \leq m \mu$, and it follows that

$$
\int_{0}^{+\infty} b(r)^{j} r^{k} e^{-b(r)} d r \leq \frac{e}{k+1}\left(\sum_{m=1}^{\infty} e^{-m} m^{j+k+1}\right) \mu^{k+1} .
$$

Corollary 4.13. There is a constant $A>0$ independent of $b$ such that

$$
A^{-1} \leq \frac{\mu(\alpha, \tau)}{\varphi(\alpha, \tau)} \leq A
$$

The next result gives additional information about $\varphi(\alpha, \tau)$ as a function of $\alpha$.

Lemma 4.14. Assume that $b$ satisfies (3-1), (3-2), and (3-3), and that $\varphi(\alpha, \tau)$ is as defined in (4-24).

(a) Let $\tau>0$. Then

$$
\frac{\partial \varphi}{\partial \alpha}(\alpha, \tau) \leq 0 \text { if } \alpha>0 \quad \text { and } \quad \frac{\partial \varphi}{\partial \alpha}(\alpha, \tau) \geq 0 \text { if } \alpha<0 .
$$

(b) There is a constant $A>0$ independent of $b$ such that for all $\alpha \in \mathbb{R}$ and all $\tau>0$,

$$
\left|\frac{\partial \varphi}{\partial \alpha}(\alpha, \tau)\right| \leq A \tau b^{\prime \prime}(\alpha) \varphi(\alpha, \tau)^{2} .
$$

(c) There is a constant $A>0$ independent of $b$ such that if $\tau>0$,

$$
\left\{\begin{array}{l}
0 \leq x_{1}<x_{2} \quad \text { implies } \varphi\left(x_{2}, \tau\right)^{-1} \leq \varphi\left(x_{1}, \tau\right)^{-1}+A \tau\left(b^{\prime}\left(x_{2}\right)-b^{\prime}\left(x_{1}\right)\right), \\
y_{1}<y_{2} \leq 0 \quad \text { implies } \varphi\left(y_{1}, \tau\right)^{-1} \leq \varphi\left(y_{2}, \tau\right)^{-1}+A \tau\left(b^{\prime}\left(y_{2}\right)-b^{\prime}\left(y_{1}\right)\right) .
\end{array}\right.
$$

Proof. Since $\varphi(\alpha, \tau)=\int_{-\infty}^{+\infty} e^{-2 \tau\left(b(\alpha+r)-b(\alpha)-r b^{\prime}(\alpha)\right)} d r$, we have

$$
\begin{aligned}
\frac{\partial \varphi}{\partial \alpha}(\alpha, \tau) & =(-2 \tau) \int_{-\infty}^{+\infty} e^{-2 \tau\left(b(\alpha+r)-b(\alpha)-r b^{\prime}(\alpha)\right)}\left(b^{\prime}(\alpha+r)-b^{\prime}(\alpha)-r b^{\prime \prime}(\alpha)\right) d r \\
= & (-2 \tau) \int_{-\infty}^{+\infty} e^{-2 \tau\left(b(\alpha+r)-b(\alpha)-r b^{\prime}(\alpha)\right)}\left(b^{\prime}(\alpha+r)-b^{\prime}(\alpha)\right) d r \\
& +2 \tau b^{\prime \prime}(\alpha) \int_{-\infty}^{+\infty} r e^{-2 \tau b_{\alpha}(r)} d r
\end{aligned}
$$




$$
\begin{aligned}
& =\int_{-\infty}^{+\infty} \frac{d}{d r}\left(e^{-2 \tau b_{\alpha}(r)}\right) d r+2 \tau b^{\prime \prime}(\alpha) \int_{-\infty}^{+\infty} r e^{-2 \tau b_{\alpha}(r)} d r \\
& =2 \tau b^{\prime \prime}(\alpha) \int_{-\infty}^{+\infty} r e^{-2 \tau b_{\alpha}(r)} d r .
\end{aligned}
$$

It follows from Proposition 4.12 that

$$
\left|\frac{\partial \varphi}{\partial \alpha}(\alpha, \tau)\right| \leq 2 \tau b^{\prime \prime}(\alpha) \int_{-\infty}^{+\infty}|r| e^{-2 \tau b_{\alpha}(r)} d r \leq A \tau b^{\prime \prime}(\alpha) \varphi(\alpha)^{2},
$$

which establishes (b). Also,

$$
\int_{-\infty}^{+\infty} r e^{-2 \tau b_{\alpha}(r)} d r=\int_{0}^{\infty} r\left(e^{-2 \tau b_{\alpha}(r)}-e^{-2 \tau b_{\alpha}(-r)}\right) d r .
$$

According to Proposition 4.6, $b_{\alpha}(r)>b_{\alpha}(-r)$ when $\alpha>0$ and $r>0$. Hence $(\partial \varphi / \partial \alpha)(\alpha, \tau)$ is positive for $\alpha<0$ and negative for $\alpha>0$, which establishes (a).

Now if $s>0$ we have

$$
-\frac{\partial \varphi}{\partial s}(s, \tau)=\left|\frac{\partial \varphi}{\partial s}(s, \tau)\right| \leq 2 \tau b^{\prime \prime}(s) \int_{-\infty}^{+\infty}|r| e^{-2 \tau b_{s}(r)} d r \leq A \tau b^{\prime \prime}(s) \varphi(s, \tau)^{2},
$$

and so

$$
\frac{\partial}{\partial s}\left(\frac{1}{\varphi(s, \tau)}\right) \leq A \tau b^{\prime \prime}(s)
$$

On the other hand, if $s<0$, we have

$$
\frac{\partial \varphi}{\partial s}(s, \tau)=\left|\frac{\partial \varphi}{\partial s}(s, \tau)\right| \leq 2 \tau b^{\prime \prime}(s) \int_{-\infty}^{+\infty}|r| e^{-2 \tau b_{s}(r)} d r \leq A \tau b^{\prime \prime}(s) \varphi(s, \tau)^{2},
$$

and so

$$
-\frac{\partial}{\partial s}\left(\frac{1}{\varphi(s, \tau)}\right) \leq A \tau b^{\prime \prime}(s)
$$

Thus if $0 \leq x_{1}<x_{2}$ we can integrate (4-28) from $x_{1}$ to $x_{2}$ and obtain

$$
\frac{1}{\varphi\left(x_{1}, \tau\right)} \leq \frac{1}{\varphi\left(x_{2}, \tau\right)} \leq \frac{1}{\varphi\left(x_{1}, \tau\right)}+A \tau\left(b^{\prime}\left(x_{2}\right)-b^{\prime}\left(x_{1}\right)\right) .
$$

If $y_{1}<y_{2}<0$ we can integrate (4-29) from $y_{1}$ to $y_{2}$ and obtain

$$
\frac{1}{\varphi\left(y_{1}, \tau\right)} \leq \frac{1}{\varphi\left(y_{2}, \tau\right)}+A \tau\left(b^{\prime}\left(y_{2}\right)-b^{\prime}\left(y_{1}\right)\right) .
$$

We need one further preliminary estimate.

Proposition 4.15. Suppose that $b$ is subcritical. Then for any $\beta, N>0$

$$
\int_{-1}^{+1} e^{-\beta|\lambda|^{-1}}\left(b(\lambda)^{-N}+\left|b^{\prime}(\lambda)\right|^{-N}+b^{\prime \prime}(\lambda)^{-N}\right) d \lambda<+\infty .
$$


Proof. If $b$ is subcritical, then according to Definition 3.2, there are constants $0<\gamma<1$ and $C>0$ such that $\left|b^{\prime}(r)\right| b(r)^{-1} \leq C|r|^{-1-\gamma}$. Integration of this inequality shows that $b(r)^{-1} \leq C_{1} \exp \left(C_{2}|r|^{-\gamma}\right)$ for some constants $C_{1}$ and $C_{2}$. It follows that for any $N \geq 0$, we have, since $\gamma<1$,

$$
\int_{-1}^{+1} e^{-\beta|\lambda|^{-1}} b(\lambda)^{-N} d \lambda \leq C_{1} \int_{-1}^{+1} e^{-\beta|\lambda|^{-1}+N C_{2}|\lambda|^{-\gamma}} d \lambda<\infty .
$$

To deal with the integral involving $\left|b^{\prime}(\lambda)\right|^{-N}$ or $b^{\prime \prime}(\lambda)^{-N}$ instead of $b(\lambda)^{-N}$, we can use the hypothesis in (3-4). We have

$$
\begin{gathered}
\frac{1}{\left|b^{\prime}(\lambda)\right|}=\frac{1}{b(\lambda)} \frac{b(\lambda)}{\left|b^{\prime}(\lambda)\right|} \leq \frac{C}{b(\lambda)} \frac{|B(\lambda)|}{b(\lambda)} \leq C\left(\sup _{|\lambda| \leq 1}|B(\lambda)|\right) \frac{1}{b(\lambda)^{2}} \leq \frac{C^{\prime}}{b(\lambda)^{2}}, \\
\frac{1}{b^{\prime \prime}(\lambda)}=\frac{1}{b(\lambda)} \frac{b(\lambda)}{\left|b^{\prime}(\lambda)\right|} \frac{\left|b^{\prime}(\lambda)\right|}{b^{\prime \prime}(\lambda)} \leq \frac{C^{2}}{b(\lambda)} \frac{B(\lambda)^{2}}{b(\lambda)^{2}} \leq C^{2}\left(\sup _{|\lambda| \leq 1} B(\lambda)^{2}\right) \frac{1}{b(\lambda)^{3}} \leq \frac{C^{\prime \prime}}{b(\lambda)^{3}} .
\end{gathered}
$$

Thus $\left|b^{\prime}(\lambda)\right|^{-N} \lesssim b(\lambda)^{-2 N}$, and $b^{\prime \prime}(\lambda)^{-N} \lesssim b(\lambda)^{-3 N}$, and we are reduced to a known case.

4.16. The key estimates. Recall that we assume $b(r)$ is given by a convex polynomial for $|r| \geq 1$, and hence that $b(r) \lesssim(1+|r|)^{N}$ for some positive integer $N$. We shall deal with functions like $b^{\prime}(\lambda)-b^{\prime}(\alpha)$ or $b^{\prime}(\lambda+\alpha)$ which then grow like $|\lambda|^{N-1}$, uniformly for $|\alpha|$ bounded. Thus let $\Phi(\lambda, \alpha)$ be any function such that if $|\alpha| \leq \alpha_{0}<\infty$, we have $|\Phi(\lambda, \alpha)| \leq C(1+|\lambda|)^{N-1}$, where $C$ can depend on $\alpha_{0}$.

Lemma 4.17. Let $m \geq n+1 \geq 1$ be positive, let $M_{0}>0$, and let $0 \leq \alpha_{0}<+\infty$. Then there is a constant $C=C\left(m, n, M_{0}, \alpha_{0}\right)<+\infty$ such that if $M \geq M_{0}$ and $|\alpha| \leq \alpha_{0}$ then

$$
\iint_{\tau>0} e^{-\tau\left(M+2 h_{\alpha}(\lambda-\alpha)\right)} \varphi(\lambda, \tau)^{-1} \tau^{m}|\Phi(\lambda, \alpha)|^{n} b^{\prime \prime}(\lambda) d \lambda d \tau<C .
$$

Proof. We can assume that $b(r) \leq C|r|^{N}$ for large $|r|$ and $b(r) \leq C r^{2}$ for small $|r|$. It follows that $\left(b^{-1}\left(\lambda^{-1}\right)\right)^{-1} \leq(C \lambda)^{1 / N}$ for $\lambda$ small, and $\left(b^{-1}\left(\lambda^{-1}\right)\right)^{-1} \leq(C \lambda)^{1 / 2}$ for $\lambda$ large. Then using Proposition 4.12 and inequality (4-13) of Proposition 4.9, we have

$$
\begin{aligned}
\varphi(\lambda, \tau)^{-1}=\left(\int_{\mathbb{R}} e^{-2 \tau b_{\lambda}(r)} d r\right)^{-1} & \leq C\left|\left\{r \in \mathbb{R} \mid b_{\lambda}(r) \leq \tau^{-1}\right\}\right|^{-1} \\
& \leq C\left(\tau^{1 / 2} b^{\prime \prime}(\lambda)^{1 / 2}+\tau b^{\prime}(\lambda)+\left(b^{-1}\left(\tau^{-1}\right)\right)^{-1}\right) \\
& \leq C\left(\tau^{1 / 2} b^{\prime \prime}(\lambda)^{1 / 2}+\tau b^{\prime}(\lambda)+\tau^{1 / 2}+\tau^{1 / N}\right) .
\end{aligned}
$$


Thus to prove Lemma 4.17, it suffices to show that each of the following three integrals is bounded by a constant depending only on $m, n, M_{0}$, and $\alpha_{0}$ :

$$
\begin{aligned}
I & =\iint_{\tau \geq 0} e^{-\tau\left[M_{0}+2 h_{\alpha}(\lambda-\alpha)\right]} \tau^{m+1 / 2} b^{\prime \prime}(\lambda)^{3 / 2}|\Phi(\lambda, \alpha)|^{n} d \lambda d \tau \\
& =C_{m} \int_{-\infty}^{+\infty} \frac{|\Phi(\lambda, \alpha)|^{n} b^{\prime \prime}(\lambda)^{3 / 2}}{\left(M_{0}+2 h_{\alpha}(\lambda-\alpha)\right)^{m+3 / 2}} d \lambda, \\
I I & =\iint_{\tau \geq 0} e^{-\tau\left(M_{0}+2 h_{\alpha}(\lambda-\alpha)\right)} b^{\prime}(\lambda) \tau^{m+1}|\Phi(\lambda, \alpha)|^{n} b^{\prime \prime}(\lambda) d \lambda d \tau \\
& =C_{m} \int_{-\infty}^{+\infty}|\Phi(\lambda, \alpha)|^{n} b^{\prime}(\lambda) b^{\prime \prime}(\lambda) /\left(M_{0}+2 h_{\alpha}(\lambda-\alpha)\right)^{m+2} d \lambda, \\
I I I & =\iint_{\tau \geq 0} e^{-\tau\left(M_{0}+2 h_{\alpha}(\lambda-\alpha)\right)} \tau^{m}|\Phi(\lambda, \alpha)|^{n} b^{\prime \prime}(\lambda)\left(\tau^{1 / 2}+\tau^{1 / N}\right) d \lambda d \tau \\
& \lesssim \int_{-\infty}^{+\infty} \frac{|\Phi(\lambda, \alpha)|^{n} b^{\prime \prime}(\lambda)}{\left(M_{0}+2 h_{\alpha}(\lambda-\alpha)\right)^{m+3 / 2}} d \lambda+\int_{-\infty}^{+\infty} \frac{|\Phi(\lambda, \alpha)|^{n} b^{\prime \prime}(\lambda)}{\left(M_{0}+2 h_{\alpha}(\lambda-\alpha)\right)^{m+1+1 / N}} d \lambda .
\end{aligned}
$$

In all three integrals, the denominator of the integrand never vanishes since $M_{0}>0$ and $h_{\alpha}(\lambda-\alpha) \geq 0$. The only issue is the convergence of the integral as $\lambda \rightarrow \pm \infty$. But for large $|\lambda|$, we assume that $b(\lambda) \sim|\lambda|^{N}$ for some $N$, and so for $|\alpha|$ bounded, $b^{\prime \prime}(\lambda) \sim|\lambda|^{N-2},\left|b^{\prime}(\lambda)\right| \sim|\lambda|^{N-1}$, and $h_{\alpha}(\lambda-\alpha) \sim|\lambda|^{N}$ for large $|\lambda|$. Thus all three integrals converge, and clearly depend continuously on $\alpha$.

Lemma 4.18. Let $m \geq 1, n \geq 0, q \geq 0, \beta_{0}>0$, and $0<\alpha_{0}<\alpha_{1}<+\infty$.

(a) If $b$ is subcritical, then there is a constant $C=C\left(m, n, q, \beta_{0}, \alpha_{1}\right)<+\infty$ such that if $\beta \geq \beta_{0}$ and if $|\alpha| \leq \alpha_{1}$, then

$$
\iint_{\tau>0} e^{-2 \tau h_{\alpha}(\lambda-\alpha)-\beta / \varphi(\lambda, \tau)} \varphi(\lambda, \tau)^{-q} \tau^{m+n}|\Phi(\lambda, \alpha)|^{n} b^{\prime \prime}(\lambda) d \lambda d \tau<C .
$$

(b) If $b$ is supercritical, there is a constant $C=C\left(m, n, q, \beta_{0}, \alpha_{0}, \alpha_{1}\right)<+\infty$ such that if $\beta \geq \beta_{0}$ and if $\alpha_{0} \leq|\alpha| \leq \alpha_{1}$, then

$$
\iint_{\tau>0} e^{-2 \tau h_{\alpha}(\lambda-\alpha)-\beta / \varphi(\lambda, \tau)} \varphi(\lambda, \tau)^{-q} \tau^{m+n}|\Phi(\lambda, \alpha)|^{n} b^{\prime \prime}(\lambda) d \lambda d \tau<C .
$$

Note that in (a) the constant $C$ is independent of $\alpha_{0}$, while in (b), the constant can depend on $\alpha_{0}$. 
Proof. First observe that if $\beta \geq \beta_{0}$ and $\beta^{\prime}=\beta / 2$, $\varphi(\lambda, \tau)^{-q} \exp \left(-\frac{\beta}{\varphi(\lambda, \tau)}\right) \leq C_{q} \beta^{-q} \exp \left(-\frac{\beta^{\prime}}{\varphi(\lambda, \tau)}\right) \leq C_{q} \beta_{0}^{-q} \exp \left(-\frac{\beta^{\prime}}{\varphi(\lambda, \tau)}\right)$, where $C_{q}$ depends only on $q$. But since the constant $C$ in either (a) or (b) is allowed to depend on $\beta_{0}$ and $q$, it suffices to consider only the case $q=0$.

Next, we study the integral in $\tau$. Put

$$
\begin{aligned}
& H(\lambda, \alpha, \beta, m+n)=\int_{0}^{\infty} \exp \left(-2 \tau h_{\alpha}(\lambda-\alpha)-\frac{\beta}{\varphi(\lambda, \tau)}\right) \tau^{m+n} d \tau \\
& \quad=\int_{b(\lambda)^{-1}}^{\infty}+\int_{h(\lambda)^{-1}}^{b(\lambda)^{-1}}+\int_{0}^{h(\lambda)^{-1}} \exp \left(-2 \tau h_{\alpha}(\lambda-\alpha)-\frac{\beta}{\varphi(\lambda, \tau)}\right) \tau^{m+n} d \tau \\
& =I+I I+I I I .
\end{aligned}
$$

It follows from Proposition 4.9 and Corollary 4.13 that

$$
\varphi(\lambda, \tau)^{-1} \geq \begin{cases}c \sqrt{\tau b^{\prime \prime}(\lambda)}, & \text { in integral } I, \\ c \tau b^{\prime}(\lambda) & \text { in integral II, } \\ c b^{-1}\left(\tau^{-1}\right)^{-1} & \text { in integral III. }\end{cases}
$$

We analyze each integral separately. We will use the inequality

$$
\int_{\kappa}^{\infty} t^{\rho} e^{-\delta t-\mu \sqrt{t}} d t \leq C_{\rho}\left(\delta+\mu^{2}\right)^{-\rho-1}\left(\exp \left[-\frac{1}{2} \kappa \delta\right]+\exp \left(-\frac{1}{2} \sqrt{\kappa} \mu\right)\right)
$$

where $\kappa, \rho, \delta, \mu>0$ and $C_{\rho}$ is a constant depending only on $\rho$.

Integral I: It follows from (4-30) that

$$
I \leq \int_{b(\lambda)^{-1}}^{+\infty} e^{-2 \tau h_{\alpha}(\lambda-\alpha)-c \beta \sqrt{\tau} \sqrt{b^{\prime \prime}(\lambda)}} \tau^{m+n} d \tau
$$

We make two estimates. We can replace the integral over $\left[b(\lambda)^{-1}, \infty\right)$ by the integral over $[0, \infty)$, and obtain

$$
I \leq C\left(h_{\alpha}(\lambda-\alpha)+b^{\prime \prime}(\lambda)\right)^{-m-n-1},
$$

On the other hand, we can replace the term $e^{-2 \tau h_{\alpha}(\lambda-\alpha)}$ in the integrand by 1 , and get

$$
I \leq C \exp \left(-\frac{c \beta}{2} \sqrt{\frac{b^{\prime \prime}(\lambda)}{b(\lambda)}}\right) b^{\prime \prime}(\lambda)^{-m-n-1} .
$$

In both cases, the constant $C$ depends on $m+n$ and $\beta$. 
Integral II: It follows from (4-30) that

$$
\begin{aligned}
I I & \leq \int_{h(\lambda)^{-1}}^{\infty} e^{-\tau\left(2 h_{\alpha}(\lambda-\alpha)+c \beta\left|b^{\prime}(\lambda)\right|\right)} \tau^{m+n} d \tau \\
& \leq \frac{C}{\left(h_{\alpha}(\lambda-\alpha)+\left|b^{\prime}(\lambda)\right|\right)^{m+n+1}} \exp \left(-\frac{2 h_{\alpha}(\lambda-\alpha)+\beta\left|b^{\prime}(\lambda)\right|}{2 h(\lambda)}\right),
\end{aligned}
$$

where the constant $C$ depends on $m+n$ and $\beta$. Hence in particular,

$$
I I \leq C\left(h_{\alpha}(\lambda-\alpha)+\left|b^{\prime}(\lambda)\right|\right)^{-m-n-1},
$$

and

$$
I I \leq C\left|b^{\prime}(\lambda)\right|^{-m-n-1} \exp \left(-\frac{\beta\left|b^{\prime}(\lambda)\right|}{2 h(\lambda)}\right)
$$

Integral III: In this case it follows from (4-30) that

$$
I I I \leq \int_{0}^{h(\lambda)^{-1}} e^{-2 h_{\alpha}(\lambda-\alpha) \tau-c \beta\left[b^{-1}\left(\tau^{-1}\right)\right]^{-1}} \tau^{m+n} d \tau .
$$

Make the substitution $b^{-1}\left(\tau^{-1}\right)=s$, so that $\tau=b(s)^{-1}$, and $d \tau=-\left(b^{\prime}(s) / b(s)^{2}\right) d s$. Then Proposition 4.3(c) and Proposition 4.4, we have $b(\lambda) \leq h(\lambda) \leq b(2 \lambda)$, so $\lambda \leq b^{-1}(h(\lambda)) \leq 2 \lambda$. Thus we get

$$
I I I \leq \int_{\lambda}^{\infty} \exp \left(-\frac{2 h_{\alpha}(\lambda-\alpha)}{b(s)}-\frac{c \beta}{s}\right) \frac{b^{\prime}(s)}{b(s)^{m+n+2}} d s
$$

Again we make two estimates. If we drop the term $-c \beta /(2 s)$ and let $b(s)^{-1}=t$, we get

$$
I I I \leq \int_{0}^{b(\lambda)^{-1}} e^{-2 h_{\alpha}(\lambda-\alpha) t} t^{m+n} d t \leq C\left(h_{\alpha}(\lambda-\alpha)+b(\lambda)\right)^{-m-n-1} .
$$

On the other hand, if we drop the term $-2 h_{\alpha}(\lambda-\alpha) / b(s)$, we get

$$
I I I \leq \int_{\lambda}^{\infty} \exp \left(-\frac{c \beta}{2 s}\right) \frac{b^{\prime}(s)}{b(s)^{m+n+2}} d s \leq \int_{0}^{\infty} \exp \left(-\frac{c \beta}{2 s}\right) \frac{b^{\prime}(s)}{b(s)^{m+n+2}} d s,
$$

which, if finite, is independent of $\lambda$.

We now return to the study of the double integral appearing in parts (a) and (b) of Lemma 4.18. Recall that when $b$ is supercritical, we require $0<\alpha_{0} \leq|\alpha| \leq \alpha_{1}$, and we must bound the integral by a constant depending on $m, n, q, \beta_{0}, \alpha_{0}$, and $\alpha_{1}$. 
Using (4-32), (4-34) and (4-36), we have

$$
\int_{-\infty}^{+\infty} H(\alpha, \beta, \lambda, m+n)|\Phi(\lambda, \alpha)|^{n} b^{\prime \prime}(\lambda) d \lambda
$$

$$
\begin{aligned}
\leq \int_{-\infty}^{+\infty} \frac{|\Phi(\lambda, \alpha)|^{n} b^{\prime \prime}(\lambda)}{\left(h_{\alpha}(\lambda-\alpha)+b^{\prime \prime}(\lambda)\right)^{m+n+1}} d \lambda & +\int_{-\infty}^{+\infty} \frac{|\Phi(\lambda, \alpha)|^{n} b^{\prime \prime}(\lambda)}{\left(h_{\alpha}(\lambda-\alpha)+\left|b^{\prime}(\lambda)\right|\right)^{m+n+1}} d \lambda \\
& +\int_{-\infty}^{+\infty} \frac{|\Phi(\lambda, \alpha)|^{n} b^{\prime \prime}(\lambda)}{\left[b(\lambda)+h_{\alpha}(\lambda-\alpha)\right]^{m+n+1}} d \lambda .
\end{aligned}
$$

We need to check that all the integrals on the right side of (4-38) converge at infinity, but this follows as in the proof of Lemma 4.17 since we are assuming that $b(\lambda) \approx|\lambda|^{N}$ for large $|\lambda|$, with $N \geq 2$ and $m \geq 1$. Also, if $\alpha \neq 0$, the denominators in these integrals never vanish and clearly depend continuously on $\alpha$. Thus in the critical case, when $|\alpha| \geq \alpha_{0}>0$, we can bound these integrals by a constant depending on $\alpha_{0}$.

In the subcritical case, we need to make estimates independent of a lower bound for $|\alpha|$. For this we use all the estimates (4-32)-(4-37), and split the integrals depending on whether $|\lambda| \geq 1$ or $|\lambda| \leq 1$. We have

$$
\int_{-\infty}^{+\infty} H(\alpha, \beta, \lambda, m+n)|\Phi(\lambda, \alpha)|^{n} b^{\prime \prime}(\lambda) d \lambda
$$

$\leq \int_{|\lambda| \geq 1} \frac{|\Phi(\lambda, \alpha)|^{n} b^{\prime \prime}(\lambda)}{\left(h_{\alpha}(\lambda-\alpha)+b^{\prime \prime}(\lambda)\right)^{m+n+1}} d \lambda+\int_{|\lambda| \leq 1} \exp \left(-\frac{c \beta}{2} \sqrt{\frac{b^{\prime \prime}(\lambda)}{b(\lambda)}}\right) \frac{\Phi(\lambda, \alpha)^{n} b^{\prime \prime}(\lambda)}{b^{\prime \prime}(\lambda)^{m+n+1}} d \lambda$

$+\int_{|\lambda| \geq 1} \frac{|\Phi(\lambda, \alpha)|^{n} b^{\prime \prime}(\lambda)}{\left(h_{\alpha}(\lambda-\alpha)+\left|b^{\prime}(\lambda)\right|\right)^{m+n+1}} d \lambda+\int_{|\lambda| \leq 1} \exp \left(-\frac{\beta\left|b^{\prime}(\lambda)\right|}{2 h(\lambda)}\right) \frac{|\Phi(\lambda, \alpha)|^{n} b^{\prime \prime}(\lambda)}{\left|b^{\prime}(\lambda)\right|^{m+n+1}} d \lambda$

$+\int_{|\lambda| \geq 1} \frac{|\Phi(\lambda, \alpha)|^{n} b^{\prime \prime}(\lambda)}{\left(b(\lambda)+h_{\alpha}(\lambda-\alpha)\right)^{m+n+1}}$

$$
+\int_{|\lambda| \leq 1}|\Phi(\lambda, \alpha)|^{n} b^{\prime \prime}(\lambda) d \lambda\left(\int_{0}^{\infty} \exp \left(-\frac{\beta}{2 s}\right) \frac{b^{\prime}(s)}{b(s)^{m+n+2}} d s\right) .
$$

The integrals for $|\lambda| \geq 1$ still converge. We need to check that the three integrals for $|\lambda| \leq 1$ converge despite the presence in the denominator of terms $b^{\prime \prime}(\lambda)^{m+n+1}$, $\left|b^{\prime}(\lambda)\right|^{m+n+1}$, and $b(\lambda)^{m+n+2}$. However, $b(\lambda) \leq \lambda^{2} b^{\prime \prime}(\lambda)$ and $h(\lambda) \leq \lambda\left|b^{\prime}(\lambda)\right|$, so in the subcritical case, the integrals converge by Proposition 4.15 .

\section{Estimates for the function $N$}

To estimate the Bergman and Szegő kernels, we shall need detailed information about the behavior of the term $N_{\alpha}(\eta, \tau)$, defined in (4-25), which appears in the 
denominator. Indeed, we will need to study this function when the real variable $\eta$ is replaced by a complex variable $z$. Note that, for each real $\alpha$,

$$
N_{\alpha}(z, \tau)=\int_{-\infty}^{+\infty} e^{2\left(r z-\tau b_{\alpha}(r)\right)} d r
$$

converges absolutely for all $z \in \mathbb{C}$ and defines an entire function. These functions are related as follows.

Proposition 5.1. For $z \in \mathbb{C}, \tau>0$, and $\alpha, \beta \in \mathbb{R}$,

$$
N_{\alpha+\beta}(z, \tau)=e^{-2 \beta z} e^{-2 \tau h_{\alpha}(\beta)} N_{\alpha}\left(z+\tau b_{\alpha}^{\prime}(\beta), \tau\right) .
$$

In particular, for $z=0$,

$$
N_{\alpha}\left(\tau b_{\alpha}^{\prime}(\beta), \tau\right)=e^{2 \tau h_{\alpha}(\beta)} \int_{-\infty}^{+\infty} e^{-2 \tau b_{\alpha+\beta}(r)} d r
$$

Proof. Since $b_{\alpha+\beta}=\left(b_{\alpha}\right)_{\beta}$, we have

$$
\begin{aligned}
N_{\alpha+\beta}(z, \tau) & =\int_{-\infty}^{+\infty} e^{2\left(r z-\tau b_{\alpha+\beta}(r)\right)} d r \\
& =\int_{-\infty}^{+\infty} \exp \left(2\left(r z-\tau b_{\alpha}(r+\beta)+\tau b_{\alpha}(\beta)+\tau r b_{\alpha}^{\prime}(\beta)\right)\right) d r \\
& =e^{2 \tau b_{\alpha}(\beta)} \int_{-\infty}^{+\infty} \exp \left(2\left(r z-\tau b_{\alpha}(r+\beta)+\tau r b_{\alpha}^{\prime}(\beta)\right)\right) d r \\
& =e^{2 \tau b_{\alpha}(\beta)} \int_{-\infty}^{+\infty} \exp \left(2\left((s-\beta) z-\tau b_{\alpha}(s)+\tau(s-\beta) b_{\alpha}^{\prime}(\beta)\right)\right) d s \\
& =e^{-2 \beta z} e^{2 \tau b_{\alpha}(\beta)-2 \tau \beta b_{\alpha}^{\prime}(\beta)} \int_{-\infty}^{+\infty} \exp \left(2\left(s z-\tau b_{\alpha}(s)+\tau s b_{\alpha}^{\prime}(\beta)\right)\right) d s \\
& =e^{-2 \beta z} e^{-2 \tau h_{\alpha}(\beta)} N_{\alpha}\left(z+\tau b_{\alpha}^{\prime}(\beta), \tau\right) .
\end{aligned}
$$

5.2. Estimates for $N_{\alpha}(z, \tau)$ for $z \in \mathbb{R}$. We begin by studying the behavior of $N(z, \tau)=N_{0}(z, \tau)$ on the real axis. When $b$ is even, we have the following simple estimate for $N_{0}(\eta, \tau)$ from below. (Recall from (4-26) that $\mu(\lambda, \tau)$ is the measure of the set where $b_{\lambda}(r) \leq \tau^{-1}$.)

Proposition 5.3. Suppose that $b \in \mathscr{C}^{\infty}(\mathbb{R})$ satisfies (3-1) and is even. Then

$$
N_{0}(\eta, \tau) \geq e^{-2}\left(\frac{e^{\mu(0, \tau) \eta}-e^{-\mu(0, \tau) \eta}}{2 \eta}\right)=e^{-2} \eta^{-1} \sinh (\mu(0, \tau) \eta) .
$$

Proof. Let $\mu=\mu(0, \tau)$. Then $\tau b(r) \leq 1$ on the interval $-\mu / 2 \leq r \leq+\mu / 2$, and so

$$
N_{0}(\eta, \tau) \geq \int_{-\mu / 2}^{+\mu / 2} e^{2 \eta r} e^{-2 \tau b(r)} d r \geq e^{-2} \int_{-\mu / 2}^{+\mu / 2} e^{2 \eta r} d r=e^{-2}\left(\frac{e^{\mu \eta}-e^{-\mu \eta}}{2 \eta}\right) .
$$


We next obtain bounds for $\eta$-derivatives of $N_{0}(\eta, \tau)$ for general convex, not necessarily even, functions $b$.

Proposition 5.4. Suppose that $b \in \mathscr{C}^{\infty}(\mathbb{R})$ satisfies (3-1). There is a constant $C>0$ such that

$$
C^{-1} e^{2 \tau h(\lambda)} \mu(\lambda, \tau) \leq N_{0}\left(\tau b^{\prime}(\lambda), \tau\right) \leq C e^{2 \tau h(\lambda)} \mu(\lambda, \tau) .
$$

Moreover, for each positive integer $n$, there is a constant $C_{n}$ such that

$$
\left|\frac{\partial^{n} N_{0}}{\partial \eta^{n}}\left(\tau b^{\prime}(\lambda), \tau\right)\right| \leq C_{n} N_{0}\left(\tau b^{\prime}(\lambda), \tau\right)\left(\mu(\lambda, \tau)^{n}+\lambda^{n}\right) .
$$

Proof. Making the change $r \rightarrow r+\lambda$, we have

$$
\frac{\partial^{n} N}{\partial \eta^{n}}(\eta, \tau)=2^{n} \int_{-\infty}^{+\infty} r^{n} e^{2(\eta r-\tau b(r))} d r=2^{n} e^{2(\eta \lambda-\tau b(\lambda))} \int_{-\infty}^{+\infty}(r+\lambda)^{n} e^{-2 \tau b_{\lambda}(r)} d r .
$$

When $n=0$, (5-4) follows from Proposition 4.12 or Corollary 4.13. If $n \geq 1$, then $|r+\lambda|^{n} \leq C_{n}\left(|r|^{n}+|\lambda|^{n}\right)$, and so

$$
\left|\frac{\partial^{n} N}{\partial \eta^{n}}(\eta, \tau)\right| \leq C_{n} e^{2(\eta \lambda-\tau b(\lambda))}\left(\int_{-\infty}^{+\infty}|r|^{n} e^{-2 \tau b_{\lambda}(r)} d r+|\lambda|^{n} \int_{-\infty}^{+\infty} e^{-2 \tau b_{\lambda}(r)} d r\right) .
$$

Then (5-5) follows from Proposition 4.12 and the inequality in (5-4).

5.5. The complex zeros of $N_{\boldsymbol{\alpha}}(z, \tau)$. We study $N_{\alpha}(z, \tau)=\int_{-\infty}^{+\infty} e^{2\left(r z-\tau b_{\alpha}(r)\right)} d r$ as a function of the complex variable $z$. Clearly $N_{\alpha}(\eta, \tau)>0$ for $\eta \in \mathbb{R}$. Our first objective is to identify a zero-free region for $N_{\alpha}(z, \tau)$ about the real axis.

Proposition 5.6. Suppose that $b \in \mathscr{C}^{\infty}(\mathbb{R})$ satisfies (3-1). There is an absolute constant $C$ such that if $\lambda \in \mathbb{R}$,

$$
\begin{aligned}
& \left|e^{-2 \lambda y i} N_{0}\left(\tau b^{\prime}(\lambda)+i y, \tau\right)-N_{0}\left(\tau b^{\prime}(\lambda), \tau\right)\right| \leq C|y| \mu(\lambda, \tau) N_{0}\left(\tau b^{\prime}(\lambda), \tau\right) \\
& \quad \text { for any } y \in \mathbb{R} \text {, and }
\end{aligned}
$$

Proof. Consider first the case when $\lambda=0$. We have

$$
\frac{\partial N_{0}}{\partial z}(z, \tau)=2 \int_{-\infty}^{+\infty} r e^{2(r z-\tau b(r))} d r
$$

Thus

$$
\begin{aligned}
\mid N_{0}(i y, \tau)- & N_{0}(0, \tau)\left|\leq \int_{0}^{y}\right| \frac{\partial N}{\partial z}(i s, \tau) \mid d s \\
& \leq 2|y| \sup _{|s| \leq y}\left|\int_{-\infty}^{+\infty} r e^{2(i r s-\tau b(r))} d r\right| \leq C|y| \mu(0, \tau) N_{0}(0, \tau),
\end{aligned}
$$


where the last inequality follows from Proposition 4.12. This gives (5-6) when $\lambda=0$.

Next, it follows from Proposition 5.1 that for any $\lambda \in \mathbb{R}$,

$$
N_{0}(z, \tau)=e^{2(\lambda z-\tau b(\lambda))} \int_{-\infty}^{+\infty} e^{2\left(\left(z-\tau b^{\prime}(\lambda)\right) r-\tau b_{\lambda}(r)\right)} d r .
$$

In particular,

$$
N_{0}\left(\tau b^{\prime}(\lambda)+i y, \tau\right)=e^{2\left(\lambda\left(\tau b^{\prime}(\lambda)+i y\right)-\tau b(\lambda)\right)} \int_{-\infty}^{+\infty} e^{2 i y r} e^{-2 \tau b_{\lambda}(r)} d r .
$$

Thus

$$
\begin{aligned}
\left|e^{-2 \lambda y i} N_{0}\left(\tau b^{\prime}(\lambda)+i y, \tau\right)-N_{0}\left(\tau b^{\prime}(\lambda), \tau\right)\right| & \\
& =e^{2\left(\lambda \tau b^{\prime}(\lambda)-\tau b(\lambda)\right) \mid}\left|\int_{-\infty}^{+\infty} e^{2 i y r} e^{-2 \tau b_{\lambda}(r)} d r-\int_{-\infty}^{+\infty} e^{-2 \tau b_{\lambda}(r)} d r\right| \\
& \leq C|y|\left|\left\{r \in \mathbb{R} \mid b_{\lambda}(r) \leq \tau^{-1}\right\}\right| e^{2\left(\lambda \tau b^{\prime}(\lambda)-\tau b(\lambda)\right)} \int_{-\infty}^{+\infty} e^{-2 \tau b_{\lambda}(r)} d r \\
& =C|y|\left|\left\{r \in \mathbb{R} \mid b_{\lambda}(r) \leq \tau^{-1}\right\}\right| N_{0}\left(\tau b^{\prime}(\lambda), \tau\right),
\end{aligned}
$$

where the inequality follows from the inequality in (5-8) applied to the function $b_{\lambda}$. This establishes (5-6) in general.

Finally, if $2 C|y| \mu(\lambda, \tau) \leq 1$, it follows that

$$
\begin{aligned}
& N_{0}\left(\tau b^{\prime}(\lambda), \tau\right) \\
& \quad \leq\left|N_{0}\left(\tau b^{\prime}(\lambda), \tau\right)-e^{-2 \lambda y i} N_{0}\left(\tau b^{\prime}(\lambda)+i y, \tau\right)\right|+\left|e^{-2 \lambda y i} N_{0}\left(\tau b^{\prime}(\lambda)+i y, \tau\right)\right| \\
& \quad \leq \frac{1}{2} N_{0}\left(\tau b^{\prime}(\lambda), \tau\right)+\left|N_{0}\left(\tau b^{\prime}(\lambda)+i y, \tau\right)\right|
\end{aligned}
$$

so $\left|N_{0}\left(\tau b^{\prime}(\lambda)+i y, \tau\right)\right| \geq \frac{1}{2} N_{0}\left(\tau b^{\prime}(\lambda), \tau\right)$. We take $\sigma=(2 C)^{-1}$ and obtain (5-7).

If we now apply Proposition 5.6 to $b=b_{\alpha}$, we obtain the following:

Lemma 5.7. Suppose that $b \in \mathscr{C}^{\infty}(\mathbb{R})$ satisfies (3-1). There are absolute constants $C>0$ and $\sigma=(2 C)^{-1}$ such that for any $\alpha, \lambda \in \mathbb{R}$,

(a) for any $y \in \mathbb{R}$,

$$
\left|e^{-2 \lambda y i} N_{\alpha}\left(\tau b_{\alpha}^{\prime}(\lambda)+i y, \tau\right)-N_{\alpha}\left(\tau b_{\alpha}^{\prime}(\lambda), \tau\right)\right| \leq C|y| \mu(\lambda+\alpha, \tau) N_{\alpha}\left(\tau b_{\alpha}^{\prime}(\lambda), \tau\right) .
$$

(b) In particular,

$$
|y| \leq \sigma \mu(\lambda+\alpha, \tau)^{-1} \quad \text { implies } \quad\left|N_{\alpha}\left(\tau b_{\alpha}^{\prime}(\lambda)+i y, \tau\right)\right| \geq \frac{1}{2} N_{\alpha}\left(\tau b_{\alpha}^{\prime}(\lambda), \tau\right) .
$$

Proof. Assertions (a) and (b) follow since the function $N_{0}(z, \tau)$ defined with respect to the function $b_{\alpha}$ is the function $N_{\alpha}(z, \tau)$ defined with respect to the function $b$, and from the identity in (4-4). 
Corollary 5.8. Suppose that $b \in \mathscr{C}^{\infty}(\mathbb{R})$ satisfies (3-1) and (3-2). There is an absolute constant $\sigma>0$ such that

$$
|y| \leq \sigma \mu(0, \tau)^{-1} \quad \text { implies } \quad\left|N_{\alpha}(\eta+i y, \tau)\right| \geq \frac{1}{2} N_{\alpha}(\eta, \tau) .
$$

In particular, if $|y| \leq \sigma \mu(0, \tau)^{-1}$, then $N_{\alpha}(\eta+i y, \tau) \neq 0$.

Proof. Under the extra hypotheses of the corollary, it follows from Lemma 4.14 that $\int_{-\infty}^{+\infty} e^{-2 \tau b_{\alpha}(r)} d r$ attains its maximum for fixed $\tau$ when $\alpha=0$. Therefore $|y| \leq \sigma \mu(0, \tau)^{-1}$ implies $|y| \leq \sigma \mu(\alpha, \tau)^{-1}$ for all $\alpha \in \mathbb{R}$. The result now follows from Lemma 5.7.

We next study the function $N_{0}(z, \tau)=N(z, \tau)$ for $\tau$ large, and show that under suitable hypotheses on $b$, the function does have zeros on the imaginary axis at height above and below the origin on the order of $\mu(0, \tau)^{-1}$. To simplify notation in the next result, we write $\mu(\tau) \equiv \mu(0, \tau)=\left|\left\{r \in \mathbb{R} \mid b(r) \leq \tau^{-1}\right\}\right|$.

Theorem 5.9. Assume that $b \in C^{\infty}(\mathbb{R})$ satisfies the conditions (3-1)-(3-4) and that $\lim _{r \rightarrow 0} \Theta(r)=0$. Then there are constants $\eta, A, B>0$ such that

$$
\left|N(z, \tau)-\frac{\sinh (\mu(\tau) z)}{z}\right| \leq \frac{1}{2}\left|\frac{\sinh (\mu(\tau) z)}{z}\right|
$$

if $\Theta(\mu(\tau))<\eta$ and if $z=x+i y \in \Omega_{1} \cup \Omega_{2}$, where

$$
\begin{aligned}
& \Omega_{1}=\left\{x+i y \in \mathbb{C}|| x \mid \leq \frac{1}{\mu(\tau)} \text { and }\left|\frac{\sin (\mu(\tau) y)}{\mu(\tau) y}\right| \geq B \Theta(\mu(\tau))\right\}, \\
& \Omega_{2}=\left\{x+i y \in \mathbb{C}\left|\frac{1}{\mu(\tau)} \leq\right| x \mid \leq \frac{A}{\mu(\tau) \Theta(\mu(\tau))} \text { and }|y| \leq \frac{3 \pi}{2 \mu(\tau)}\right\} .
\end{aligned}
$$

Proof. Write

$$
\begin{aligned}
N(z, \tau) & =\int_{2|r| \leq \mu(\tau)} e^{2 z r} d r+\int_{2|r| \leq \mu(\tau)} e^{2 z r}\left(e^{-2 \tau b(r)}-1\right) d r+\int_{2|r| \geq \mu(\tau)} e^{2(z r-\tau b(r))} d r \\
& =I(z, \tau)+I I(z, \tau)+I I I(z, \tau) .
\end{aligned}
$$

Observe that $\left.I(z, \tau)=\left(e^{z \mu(\tau)}-e^{-z \mu(\tau)}\right) /(2 z)\right)=\sinh (\mu(\tau) z) / z$.

We now estimate $|I I(z, \tau)|$ and $|I I I(z, \tau)|$. Write $z=x+i y$. Since $2 \tau b(r) \geq 0$, it follows that $0 \leq 1-e^{-2 \tau b(r)} \leq 2 \tau b(r)$. Thus since $B(s)=\int_{0}^{s} b(r) d r$, we have

$$
|I I(z, \tau)| \leq \int_{2|r| \leq \mu(\tau)} e^{2|x| r}(2 \tau b(r)) d r \leq 4 \tau e^{|x| \mu(\tau)} B\left(\frac{1}{2} \mu(\tau)\right) .
$$

Next, for $r \geq \mu(\tau) / 2$, since $\tau b(\mu(\tau) / 2)=1$, we have

$$
\tau b(r) \geq \tau b\left(\frac{1}{2} \mu(\tau)\right)+\tau\left(r-\frac{1}{2} \mu(\tau)\right) b^{\prime}\left(\frac{1}{2} \mu(\tau)\right)=1+\tau\left(r-\frac{1}{2} \mu(\tau)\right) b^{\prime}\left(\frac{1}{2} \mu(\tau)\right),
$$


so $\tau b(r+\mu(\tau) / 2) \geq 1+\tau r b^{\prime}(\mu(\tau) / 2)$. Thus for $|x| \leq 1 / 2 \tau b^{\prime}(\mu(\tau) / 2)$, we have

$$
\begin{aligned}
|I I I(z, \tau)| & \leq 2 \int_{\mu(\tau) / 2}^{\infty} e^{2(r|x|-\tau b(r))} d r=2 \int_{0}^{\infty} e^{2((r+\mu(\tau) / 2)|x|-\tau b(r+\mu(\tau) / 2))} d r \\
& \leq e^{\mu(\tau)|x|} B^{\prime}(\mu(\tau) / 2) \int_{0}^{\infty} e^{2 r\left(|x|-\tau b^{\prime}(\mu(\tau) / 2)\right)} d r \leq \frac{4 e^{|x| \mu(\tau)}}{\tau b^{\prime}(\mu(\tau) / 2)} .
\end{aligned}
$$

Since $B^{\prime}(\mu(\tau) / 2)=b(\mu(\tau) / 2)=\tau^{-1}$, it follows from these estimates that when $|x| \leq \tau / 2 b^{\prime}(\mu(\tau) / 2)$, we have

$$
\begin{aligned}
\left|N(z, \tau)-\frac{\sinh (\mu(\tau) z)}{z}\right| & \leq 4 e^{|x| \mu(\tau)}\left(\tau B(\mu(\tau) / 2)+\frac{1}{\tau b^{\prime}(\mu(\tau) / 2)}\right) \\
& \leq C_{1} \mu(\tau) e^{|x| \mu(\tau)} \Theta(\mu(\tau)),
\end{aligned}
$$

where $C_{1}$ involves the constant appearing in (3-4). (We are using the fact that $\Theta(t)$ is increasing for small $t$.) It follows that the estimate in (5-9) holds provided that

$$
2 C_{1}|z| \mu(\tau) e^{\mu(\tau)|x|} \Theta(\mu(\tau)) \leq|\sinh (\mu(\tau) z)| .
$$

Note that

$|\sinh (\mu(\tau) z)|$

$$
=\left(\sinh ^{2}(\mu(\tau) x)+\sin ^{2}(\mu(\tau) y)\right)^{1 / 2} \geq \frac{1}{\sqrt{2}}(|\sinh (\mu(\tau) x)|+|\sin (\mu(\tau) y)|) .
$$

If $z \in \Omega_{1}$, then $|x| \mu(\tau) \leq 1$, and so $2 C_{1} \Theta(\mu(\tau))(1+2|x| \mu(\tau)) \leq 6 C_{1} \Theta(\mu(\tau))$. If $z \in \Omega_{2}$, then $1 \leq|x| \mu(\tau)$ and $|x| \mu(\tau) \Theta(\mu(\tau)) \leq A$, so

$$
2 C_{1} \Theta(\mu(\tau))(1+2|x| \mu(\tau)) \leq 6 C_{1}|x| \mu(\tau) \Theta(\mu(\tau) / 2) \leq 6 C_{1} A .
$$

It follows that

$$
\left.\begin{array}{rl}
\Theta(\mu(\tau))+A & \leq \epsilon\left(6 C_{1}\right)^{-1} \\
z & \in \Omega_{1} \cup \Omega_{2}
\end{array}\right\} \quad \text { implies } \quad 2 C_{1} \Theta(\mu(\tau))(1+2|x| \mu(\tau)) \leq \epsilon .
$$

Suppose $x+i y \in \Omega_{1}$. Since $t \geq 0$ implies $t e^{t} \leq(1+2 t) \sinh (t)$, it follows from (5-11) that if $\Theta(\mu(\tau))+A \leq \epsilon\left(6 C_{1}\right)^{-1}$,

$$
\begin{aligned}
2 C_{1}|x| \mu(\tau) e^{|x| \mu(\tau)} \Theta(\mu(\tau)) & \leq 2 C_{1} \Theta(\mu(\tau))(1+2 \mu(\tau)|x|) \sinh (|x| \mu(\tau)) \\
& \leq \epsilon \sinh (\mu(\tau)|x|) .
\end{aligned}
$$

Also, since $\mu(\tau)|x| \leq 1$ and $|\sin (\mu(\tau) y) /(\mu(\tau) y)| \geq B \Theta(\mu(\tau))$, if $\epsilon B \geq 2 C_{1} e^{2}$, it follows that

(5-13) $2 C_{1} \mu(\tau)|y| e^{2 \mu(\tau)|x|} \Theta(\mu(\tau)) \leq 2 C_{1} e^{2} B^{-1}|\sin (\mu(\tau) y)| \leq \epsilon|\sin (\mu(\tau) y)|$.

Thus (5-12) and (5-13) show that if $\Theta(\mu(\tau))+A \leq \epsilon\left(6 C_{1}\right)^{-1}$, if $\epsilon B \geq 2 C_{1} e^{2}$, and if $2 \sqrt{2} \epsilon<1$, then (5-10) holds for all $x+i y \in \Omega_{1}$. 
Now suppose $x+i y \in \Omega_{2}$. Using (5-11) again, we have

$$
\begin{aligned}
2 C_{1}|x| \mu(\tau) e^{|x| \mu(\tau)} \Theta(\mu(\tau)) & \leq 2 C_{1} \Theta(\mu(\tau))(1+2 \mu(\tau)|x|) \sinh (|x| \mu(\tau)) \\
& \leq \epsilon \sinh (\mu(\tau)|x|)
\end{aligned}
$$

We also have $|y| \leq 3 \pi|x| / 2<5|x|$, and so

$$
\begin{aligned}
2 C_{1} \mu(\tau)|y| e^{2 \mu(\tau)|x|} \Theta(\mu(\tau)) & \leq 5 \cdot 2 C_{1}|x| \mu(\tau) e^{|x| \mu(\tau)} \Theta(\mu(\tau)) \\
& \leq 5 \epsilon \sinh (\mu(\tau)|x|) .
\end{aligned}
$$

Thus (5-14) and (5-15) show that if $\Theta(\mu(\tau))+A \leq \epsilon\left(6 C_{1}\right)^{-1}$ and if $6 \sqrt{2} \epsilon<1$, then (5-10) holds for all $x+i y \in \Omega_{2}$.

Thus we can choose, for example, $\epsilon=10^{-2}$, and then set $A=\epsilon\left(6 C_{1}\right)^{-1} / 2$, $B=4 \epsilon^{-1} C_{1} e^{2}$, and $\eta=\epsilon\left(6 C_{1}\right)^{-1} / 2$. With these choices, the estimate (5-9) holds for all $z \in \Omega_{1} \cup \Omega_{2}$.

Corollary 5.10. Assume that $b$ satisfies the hypotheses of Theorem 5.9. Then there exists $\mu_{0}>0$ and $c>0$ such that the following hold if $\mu(\tau) \leq \mu_{0}$ :

(a) The function $N(z, \tau)$ has no zeros in the regions

$$
\left\{x+i y \in \mathbb{C}\left|\frac{1}{\mu(\tau)} \leq\right| x\left|\leq \frac{A}{\mu(\tau) \Theta(\mu(\tau))},\right| y \mid \leq \frac{3 \pi}{2 \mu(\tau)}\right\},
$$

and in these regions,

$$
|N(x+i y, \tau)| \geq c \frac{\sinh (\mu(\tau)|x|)}{|x|} .
$$

(b) The function $N(z, \tau)$ has precisely two zeros in the region

$$
\left\{x+i y \in \mathbb{C}|| x\left|\leq \frac{1}{\mu(\tau)}, 0 \leq\right| y \mid \leq \frac{3 \pi}{2 \mu(\tau)}\right\} .
$$

These zeros are complex conjugate and purely imaginary, and can be written $\pm i \sigma / \mu(\tau)$ where $3 \pi / 4<\sigma<5 \pi / 4$. Moreover, for $|x| \leq 1 / \mu(\tau)$, we have

$$
\left|N\left(x \pm i \frac{3 \pi}{2 \mu(\tau)}, \tau\right)\right| \geq c \frac{\sinh (\mu(\tau)|x|)+1}{|x|+1} .
$$

Proof. The holomorphic function $z^{-1} \sinh (\mu(\tau) z)$ has only simple zeros located at the points $\left\{n \pi i \mu(\tau)^{-1}\right\}$ for $n= \pm 1, \pm 2, \ldots$ The existence and absence of zeros for the function $N(z, \tau)$ then follows from Rouché's theorem and the estimates of Theorem 5.9. Since $N(z, \tau)=N(-z, \tau)=\overline{N(\bar{z}, \tau)}$, it follows that the zeros are purely imaginary. 
5.11. The complex zeros of $\varphi(\alpha, w)$. Recall that $\varphi(\alpha, \tau)=\int_{-\infty}^{\infty} e^{-2 \tau b_{\alpha}(r)} d r$ from (4-24). If we replace the real variable $\tau$ by a complex variable $w=\tau+i s$, then

$$
\varphi(\alpha, w)=\int_{-\infty}^{\infty} e^{-2 w b_{\alpha}(r)} d r
$$

is a holomorphic function of $w$ in the half-plane $\Re \mathrm{e}(w)>0$. Clearly $\varphi(\alpha, w)>0$ if $w$ is real. We have $(\partial / \partial s)(\varphi(\alpha, \tau+i s))=-2 i \int_{-\infty}^{\infty} b_{\alpha}(r) e^{-2(\tau+i s) b_{\alpha}(r)} d r$, and so by Proposition 4.12,

$$
\begin{aligned}
\left|\frac{\partial}{\partial s}(\varphi(\alpha, \tau+i s))\right| & \leq 2 \int_{-\infty}^{\infty} b_{\alpha}(r) e^{-2 \tau b_{\alpha}(r)} d r \\
& \leq C \tau^{-1} \int_{-\infty}^{\infty} e^{-2 \tau b_{\alpha}(r)} d r=C \tau^{-1} \varphi(\alpha, \tau) .
\end{aligned}
$$

Thus $|\varphi(\alpha, \tau+i s)-\varphi(\alpha, \tau)| \leq C|s| \tau^{-1} \varphi(\alpha, \tau)$. This proves the following result.

Lemma 5.12. There is a constant $\sigma>0$ such that if $|s| \leq \sigma \tau$, then

$$
|\varphi(\alpha, \tau+i s)-\varphi(\alpha, \tau)| \leq \frac{1}{2} \varphi(\alpha, \tau) .
$$

In particular, the holomorphic function $\varphi(\alpha, w)$ has no zeros in the angular region about the positive real axis $\{w \in \mathbb{C}|| \Im \mathrm{m}(w) \mid \leq \sigma \Re \mathrm{e}(w), w \neq 0\}$.

\section{Proof of Theorem 3.4}

Recall that

(3-8) $\mathscr{B}_{\rho}(z, w)=\frac{2^{1-\rho}}{4 \pi^{2} \Gamma(1-\rho)} \iint_{\tau>0} e^{\eta\left(z_{1}+\bar{w}_{1}\right)+i \tau\left(z_{2}-\bar{w}_{2}\right)} \frac{\tau^{1-\rho}}{\int_{\mathbb{R}} e^{2(\eta r-\tau b(r))} d r} d \eta d \tau$.

In this section, we establish the absolute convergence of this integral, and identify it with the Bergman and Szegő kernels.

6.1. Proof of absolute convergence (part (a)). Let $C=C(\rho)$ denote a constant that depends on $\rho$ and may change from one line to the next. Then with $z_{1}=x+i y$, $z_{2}=t+i b(x)+i h, w_{1}=u+i v$, and $w_{2}=s+i b(u)+i k$,

$$
\begin{aligned}
& \left|\partial_{z_{1}}^{a} \partial_{\bar{w}_{1}}^{b} \partial_{z_{2}}^{c} \partial_{\bar{w}_{2}}^{d} \Re_{\rho}\left(\left(z_{1}, z_{2}\right),\left(w_{1}, w_{2}\right)\right)\right| \\
& \leq C \iint_{\tau>0} e^{\eta(x+u)-\tau(b(x)+b(u))-\tau(h+k)} \frac{|\eta|^{a+b} \tau^{c+d+1-\rho}}{\int_{\mathbb{R}} e^{2(r \eta-\tau b(r))} d r} d \eta d \tau \\
& =C \iint_{\tau>0} e^{-\tau\left(b(x)+b(u)-2 b\left(\frac{x+u}{2}\right)+(h+k)\right)} \frac{\eta^{a+b} \tau^{c+d+1-\rho}}{\int_{\mathbb{R}} e^{2\left(\eta r-\tau\left(b\left(r+\frac{x+u}{2}\right)-b\left(\frac{x+u}{2}\right)-r b^{\prime}\left(\frac{x+u}{2}\right)\right)\right)} d r} d \eta d \tau .
\end{aligned}
$$


If we let $\alpha=(x+u) / 2, A=b(x)+b(u)-2 b(\alpha)$, and $\delta=h+k$, then

$$
\left|\partial_{z_{1}}^{a} \partial_{\bar{w}_{1}}^{b} \partial_{z_{2}}^{c} \partial_{\bar{w}_{2}}^{d} \mathscr{B}_{\rho}(z, w)\right| \leq C \iint_{\tau \geq 0} e^{-\tau(A+\delta)} \frac{\eta^{a+b} \tau^{c+d+1-\rho}}{\int_{\mathbb{R}} e^{2\left(\eta r-\tau b_{\alpha}(r)\right)} d r} d \eta d \tau .
$$

We need to show that this last integral converges for $A+\delta>0$.

However, this follows easily from Lemma 4.17. Let $a+b+c+d+2-\rho=m$ and $a+b=n$, such that $m \geq n+1 \geq 1$. Make the change of variables $\eta \rightarrow \tau b_{\alpha}^{\prime}(\lambda)$ and use Proposition 4.11. Then we have

$$
\begin{aligned}
&\left|\partial_{z_{1}}^{a} \partial_{\bar{w}_{1}}{ }_{z_{2}} \partial_{z_{2}}^{c} \partial_{\bar{w}_{2}}^{d} \Re_{\rho}(z, w)\right| \\
& \quad \leq C \iint_{\tau \geq 0} e^{-\tau[A+\delta]} e^{-2 \tau h_{\alpha}(\lambda)} \frac{\tau^{m} b_{\alpha}^{\prime}(\lambda)^{n}}{\int_{\mathbb{R}} e^{-2 \tau b_{\alpha+\lambda}(r)} d r} b_{\alpha}^{\prime \prime}(\lambda) d \lambda d \tau \\
& \quad \leq C \iint_{\tau \geq 0} e^{-\tau[A+\delta]} e^{-2 \tau h_{\alpha}(\lambda-\alpha)} \frac{\tau^{m} b_{\alpha}^{\prime}(\lambda-\alpha)^{n}}{\int_{\mathbb{R}} e^{-2 \tau b_{\lambda}(r)} d r} b^{\prime \prime}(\lambda) d \lambda d \tau \\
& \quad=C \iint_{\tau \geq 0} e^{-\tau[A+\delta]+2 \tau h_{\alpha}(\lambda-\alpha)} \varphi(\lambda, \tau)^{-1} \tau^{m}\left[b^{\prime}(\lambda)-b^{\prime}(\alpha)\right]^{n} b^{\prime \prime}(\lambda) d \lambda d \tau \\
& \quad<C(\rho, m, n, A+\delta, \alpha),
\end{aligned}
$$

where the last inequality is the content of Lemma 4.17.

6.2. Proof of part (b). The weighted Bergman spaces $B_{\rho}^{2}(\Omega)$ for $\rho<1$ are defined in Section 2.1. We want to show that the corresponding Bergman kernel is given by the function $\mathscr{B}_{\rho}(z, w)$.

A function $f \in L_{\rho}^{2}(\Omega)$ is holomorphic if and only if $f$ satisfies (in the sense of distributions) the partial differential equations

$$
\left(\frac{\partial}{\partial x}+i \frac{\partial}{\partial y}\right)[f]=0 \quad \text { and } \quad\left(\frac{\partial}{\partial t}+i \frac{\partial}{\partial s}\right)[f]=0 .
$$

If we make the nonsingular change of variables

$$
\Omega \ni(x+i y, t+i s) \rightarrow(x, y, t, s-b(x))=(x, y, t, h),
$$

the domain $\Omega$ is mapped to the domain $\mathbb{R}_{+}^{4}=\left\{(x, y, t, h) \in \mathbb{R}^{4} \mid h>0\right\}$, and in the new coordinates, equations (6-1) become

$$
\left(\frac{\partial}{\partial x}-b^{\prime}(x) \frac{\partial}{\partial h}+i \frac{\partial}{\partial y}\right)[f]=0 \quad \text { and } \quad\left(\frac{\partial}{\partial t}+i \frac{\partial}{\partial h}\right)[f]=0 .
$$

These in turn are equivalent to the equations

$$
\bar{Z}_{1}[f]=\frac{\partial f}{\partial x}+i\left(\frac{\partial f}{\partial y}-b^{\prime}(x) \frac{\partial f}{\partial t}\right)=0 \quad \text { and } \quad \bar{Z}_{2}[f]=\frac{\partial f}{\partial t}+i \frac{\partial f}{\partial h}=0 .
$$


Thus if we use coordinates $(x, y, t, h)$, the space $B_{\rho}^{2}(\Omega)$ is identified with the space of functions $B_{\rho}^{2}\left(\mathbb{R}_{+}^{4}\right) \subset L^{2}\left(\mathbb{R}_{+}^{4} ; h^{-\rho} d x d y d t d h\right)=L_{\rho}^{2}\left(\mathbb{R}_{+}^{4}\right)$, which satisfy the two equations in (6-3) in the sense of distributions.

In analogy with (2-6), define a partial Fourier transform $\mathscr{F}$ and its inverse by

$$
\begin{aligned}
\mathscr{F}[f](x, \eta, \tau, h) & =\iint_{\mathbb{R}^{2}} e^{-2 \pi i(y \eta+t \tau)} f(x, y, t, h) d y d t, \\
\mathscr{F}^{-1}[f](x, y, t, h) & =\iint_{\mathbb{R}^{2}} e^{+2 \pi i(y \eta+t \tau)} f(x, \eta, \tau, h) d \eta d \tau
\end{aligned}
$$

for $f \in \mathscr{Y}\left(\mathbb{R}^{4}\right)$, the Schwartz space. Then $\mathscr{F}$ extends to an isometry of $L^{2}\left(\mathbb{R}^{4}\right)$. Also define a multiplication operator $M$ and its inverse by

$$
\begin{aligned}
\mathcal{M}[g](x, \eta, \tau, h) & =e^{-2 \pi(\eta x-\tau b(x)-\tau h)} g(x, \eta, \tau, h), \\
\mathcal{M}^{-1}[g](x, \eta, \tau, h) & =e^{+2 \pi(\eta x-\tau b(x)-\tau h)} g(x, \eta, \tau, h) .
\end{aligned}
$$

Let $\widetilde{L}_{\rho}^{2}\left(\mathbb{R}_{+}^{4}\right)$ denote the weighted space

$$
L^{2}\left(\mathbb{R}_{+}^{4}, \Gamma(1-\rho)^{-1} e^{+4 \pi(\eta x-\tau b(x)-\tau h)} h^{-\rho} d x d \eta d \tau d h\right) .
$$

Then $\mu: L_{\rho}^{2}\left(\mathbb{R}_{+}^{4}\right) \rightarrow \widetilde{L}_{\rho}^{2}\left(\mathbb{R}_{+}^{4}\right)$ is an isometry. It is now easy to check that

$$
\bar{Z}_{1}=\mathscr{F}^{-1} \mathcal{M}^{-1} \frac{\partial}{\partial x} \mathcal{M} \mathscr{F} \quad \text { and } \quad \bar{Z}_{2}=i \mathscr{F}^{-1} \mathcal{M}^{-1} \frac{\partial}{\partial h} \mathcal{M} \mathscr{F}
$$

Thus the space $B_{\rho}^{2}\left(\mathbb{R}_{+}^{4}\right)$ is conjugate via the isometry $\mathcal{M}$ to the subspace $\widetilde{B}_{\rho}^{2}\left(\mathbb{R}_{+}^{4}\right)$ of the weighted Hilbert space $\widetilde{L}_{\rho}^{2}\left(\mathbb{R}_{+}^{4}\right)$ annihilated by the operators $\partial / \partial x$ and $\partial / \partial h$. Of course, $\widetilde{B}_{\rho}^{2}\left(\mathbb{R}_{+}^{4}\right)$ is just the space of functions in $\widetilde{L}_{\rho}^{2}\left(\mathbb{R}_{+}^{4}\right)$ that are independent of $x$ and $h$. Moreover, if $\mathscr{P}_{\rho}$ is the orthogonal projection of $\widetilde{L}_{\rho}^{2}\left(\mathbb{R}_{+}^{4}\right)$ onto this subspace, then

$$
\mathbf{B}_{\rho}=\mathscr{F}^{-1} \mathcal{M}^{-1} \mathscr{P}_{\rho} \mathcal{M} \mathscr{F} .
$$

To understand $\widetilde{B}_{\rho}^{2}\left(\mathbb{R}_{+}^{4}\right)$, suppose $\phi \in \widetilde{L}_{\rho}^{2}\left(\mathbb{R}_{+}^{4}\right)$ is independent of $x$ and $h$. Then

$$
\begin{aligned}
\|\phi\|_{\widetilde{L}_{\rho}^{2}\left(\mathbb{R}_{+}^{4}\right)}^{2} & =\iint_{\mathbb{R}^{2}}|\phi(\eta, \tau)|^{2}\left(\iint_{h>0} e^{4 \pi(\eta x-\tau b(x)-\tau h)} h^{-\rho} d h d x\right) d \eta d \tau \\
& =\Gamma(1-\rho) \iint|\phi(\eta, \tau)|^{2}(4 \pi \tau)^{\rho-1} N(2 \pi \eta, 2 \pi \tau) d \eta d \tau,
\end{aligned}
$$

where $N(\eta, \tau)=N_{0}(\eta, \tau)$ as defined in (4-25). Since $N(\eta, \tau)=+\infty$ if $\tau \leq 0$, it follows that if $\|\phi\|_{\widetilde{L}_{\rho}^{2}\left(\mathbb{R}_{+}^{4}\right)}<+\infty$, then $\phi$ is supported in the set $\left\{(\eta, \tau) \in \mathbb{R}^{2} \mid \tau \geq 0\right\}$. Thus $\widetilde{B}_{\rho}^{2}\left(\mathbb{R}_{+}^{4}\right)$ is identified with the set of functions $\phi(\eta, \tau)$ supported where $\tau \geq 0$ such that $\iint|\phi(\eta, \tau)|^{2} \tau^{\rho-1} N(2 \pi \eta, 2 \pi \tau) d \eta d \tau<+\infty$. 
For each fixed $(\eta, \tau) \in \mathbb{R}_{+}^{2}$, let

$$
\widetilde{L}_{\rho, \eta, \tau}^{2}\left(\mathbb{R}_{+}^{2}\right)=L^{2}\left(\mathbb{R}_{+}^{2}, \Gamma(1-\rho)^{-1} e^{4 \pi(\eta x-\tau b(x)-\tau h)} h^{-\rho} d x d h\right) .
$$

Then we can regard $\widetilde{L}_{\rho}^{2}\left(\mathbb{R}_{+}^{4}\right)$ as the space of functions $g$ defined on $\mathbb{R}_{+}^{2}$ so that $g(\eta, \tau) \in \widetilde{L}_{\rho, \eta, \tau}^{2}\left(\mathbb{R}_{+}^{2}\right)$ and so that $\|g(\eta, \tau)\|_{L_{\rho, \eta, \tau}^{2}\left(\mathbb{R}_{+}^{2}\right)}$ is square-integrable with respect to the measure $d \eta d \tau$. With this interpretation, $\widetilde{B}_{\rho}^{2}\left(\mathbb{R}_{+}^{4}\right)$ is the set of squareintegrable functions on $\mathbb{R}_{+}^{2}$ with values in $\widetilde{L}_{\rho, \eta, \tau}^{2}\left(\mathbb{R}_{+}^{2}\right)$ whose values are constant functions.

Let $\mathbf{0}$ and $\mathbf{1}$ be the functions of $(x, h)$ taking on the constant values 0 and 1 . Then the orthogonal projection of $\widetilde{L}_{\rho, \eta, \tau}^{2}\left(\mathbb{R}_{+}^{2}\right)$ onto the subspace of constants is given by

$$
\mathscr{P}_{\rho, \eta, \tau}[g](x, h)= \begin{cases}\mathbf{0} & \text { if } \tau<0, \\ \|\mathbf{1}\|_{\widetilde{L}_{\rho, \eta, \tau}^{2}\left(\mathbb{R}_{+}^{2}\right)}^{2}\langle g, \mathbf{1}\rangle_{\widetilde{L}_{\rho, \eta, \tau}^{2}\left(\mathbb{R}_{+}^{2}\right)} \mathbf{1} & \text { if } \tau \geq 0,\end{cases}
$$

and if $g \in \widetilde{L}_{\rho}^{2}\left(\mathbb{R}_{+}^{4}\right)$, then

$$
\mathscr{P}_{\rho}[g](x, \eta, \tau, h)=\mathscr{P}_{\rho, \eta, \tau}\left[g_{\eta, \tau}\right](x, h),
$$

where $g_{\eta, \tau}(x, h)=g(x, \eta, \tau, h)$. But

(6-10) $\|\mathbf{1}\|_{\widetilde{L}_{\rho, \eta, \tau}^{2}\left(\mathbb{R}_{+}^{2}\right)}^{2}=\iint_{\mathbb{R}_{+}^{2}} e^{4 \pi(\eta x-\tau b(x)-\tau h)} h^{-\rho} d x d h=(4 \pi \tau)^{\rho-1} N(2 \pi \eta, 2 \pi \tau)$.

Thus from (6-8), if $\tau \geq 0$,

(6-11) $\mathscr{P}_{\eta, \tau}[g](x, h)=\frac{(4 \pi \tau)^{1-\rho}}{\Gamma(1-\rho) N(2 \pi \eta, 2 \pi \tau)} \iint_{\mathbb{R}_{+}^{2}} g(r, k) e^{4 \pi(\eta r-\tau b(r)-\tau k)} d r d k$.

Hence it follows from (6-9) that $P[g](x, \eta, \tau, h)=0$ if $\tau<0$, and, if $\tau \geq 0$,

(6-12) $\quad P[g](x, \eta, \tau, h)$

$$
=\frac{(4 \pi \tau)^{1-\rho}}{\Gamma(1-\rho) N(2 \pi \eta, 2 \pi \tau)} \iint_{\mathbb{R}_{+}^{2}} g(r, \eta, \tau, k) e^{4 \pi(\eta r-\tau b(r)-\tau k)} k^{-\rho} d r d k .
$$

We can now compute $\mathbf{B}_{\rho}=\mathscr{F}^{-1} \mathcal{M}^{-1} \mathscr{P}_{\rho} \mathcal{M} \mathscr{F}$. We have

$$
\begin{gathered}
\mathbf{B}_{\rho}[f](x, y, t, h)=\iint e^{2 \pi i(\eta y+t \tau)} \mathcal{M}^{-1} \mathscr{P}_{\rho} \mathcal{M} \mathscr{F}[f](x, \eta, \tau, h) d \eta d \tau \\
=\iint e^{2 \pi i(\eta y+t \tau)+2 \pi(\eta x-\tau b(x)-\tau h)} \mathscr{P}_{\rho} \mathcal{M} \mathscr{F}[f](x, \eta, \tau, h) d \eta d \tau
\end{gathered}
$$




$$
\begin{aligned}
& =\frac{1}{\Gamma(1-\rho)} \iiint_{\tau \geq 0, k \geq 0} e^{2 \pi i(\eta y+t \tau)+2 \pi(\eta x-\tau b(x)-\tau h)+4 \pi(\eta u-\tau b(u)+\tau k)} \\
& \quad \times \frac{(4 \pi \tau)^{1-\rho}}{N(2 \pi \eta, 2 \pi \tau)} M \mathscr{F}[f](u, \eta, \tau, k) k^{-\rho} d u d k d \eta d \tau \\
& =\frac{1}{\Gamma(1-\rho)} \iiint_{\tau \geq 0, k \geq 0} e^{2 \pi i(\eta y+t \tau)+2 \pi(\eta x-\tau b(x)-\tau h)+4 \pi(\eta u-\tau b(u)+\tau k)-2 \pi(\eta u-\tau b(u)-\tau k)} \\
& \quad \times \frac{(4 \pi \tau)^{1-\rho}}{N(2 \pi \eta, 2 \pi \tau)} \mathscr{F}[f](u, \eta, \tau, k) k^{-\rho} d u d k d \eta d \tau \\
& =\frac{1}{\Gamma(1-\rho)} \iiint_{\tau \geq 0, k \geq 0} e^{2 \pi i(\eta(y-v)+\tau(t-s))} e^{+2 \pi(\eta(x+u)-\tau(b(x)+b(u))-\tau(h+k))} \\
& \times \frac{(4 \pi \tau)^{1-\rho}}{N(2 \pi \eta, 2 \pi \tau)} f(u, v, s, k) k^{-\rho} d u d k d \eta d \tau d v d v \\
& =\frac{1}{\Gamma(1-\rho)} \iint_{k \geq 0} f(u, v, s, k) \mathscr{B}_{\rho}((x, y, t, h),(u, v, s, k)) k^{-\rho} d u d v d s d k,
\end{aligned}
$$

where

$$
\begin{aligned}
& \mathscr{B}_{\rho}((x, y, t, h),(u, v, s, k)) \\
& =(2 \pi)^{-2} 2^{1-\rho} \iint_{\tau \geq 0} e^{i(\eta(y-v)+\tau(t-s))} e^{+(\eta(x+u)-\tau(b(x)+b(u))-\tau(h+k))} \frac{\tau^{1-\rho}}{N(\eta, \tau)} d \eta d \tau \\
& =(2 \pi)^{-2} 2^{1-\rho} \iint_{\tau \geq 0} e^{\eta((x+i y)+(u-i v))} e^{i \tau((t+i(b(x)+h))-(s-i(b(u)+k)))} \frac{\tau^{1-\rho}}{N(\eta, \tau)} d \eta d \tau .
\end{aligned}
$$

If we go back to complex coordinates in which $z_{1}=x+i y, w_{1}=r+i s$, $z_{2}=t+i b(x)+i h$, and $w_{2}=w+i b(r)+i k$, the formula for the projection kernel becomes

$$
\mathscr{B}_{\rho}(z, w)=\frac{2^{1-\rho}}{4 \pi^{2}} \iint_{\tau>0} e^{\eta\left(z_{1}+\bar{w}_{1}\right)} e^{i \tau\left(z_{2}-\bar{w}_{2}\right)} \frac{\tau^{1-\rho}}{N(\eta, \tau)} d \eta d \tau .
$$

This completes the proof of part (b) of Theorem 3.4.

6.3. Proof of part (c). The proof of the formula for the Szegó kernel $\mathscr{S}$ is very similar to that for the Bergman kernels for $\rho<1$, except that in view of the discussion in Section 2.3, the analysis is carried out in $L^{2}(\partial \Omega)=L^{2}\left(\mathbb{R}^{3}\right)$ instead of $L^{2}\left(\mathbb{R}_{+}^{4}\right)$. Thus we shall be brief. According to Corollary 2.6, we can identify the space $\mathscr{H}^{2}(\Omega)$ with the closed subspace of functions $f(x, y, t)$ in $L^{2}\left(\mathbb{R}^{3}\right)$ that are annihilated in the sense of distributions by $\partial / \partial x+i \partial / \partial y-i b^{\prime}(x) \partial / \partial t$. According to Proposition 2.5, the partial Fourier transform $\mathscr{F}$ defined in (2-6) is an 
isometry on $L^{2}\left(\mathbb{R}^{3}\right)$ and identifies $\mathscr{H}^{2}(\Omega)$ with the space of functions $\hat{f}(x, \eta, \tau)$ satisfying $(\partial / \partial x)\left(e^{-2 \pi(\eta x-\tau b(x))} \hat{f}(x, \eta, \tau)\right)=0$. Therefore if $\mathcal{M}[\hat{f}](x, \eta, \tau)=$ $e^{-2 \pi(\eta x-\tau b(x))} \hat{f}(x, \eta, \tau)$, it follows that $\mathscr{H}^{2}(\Omega)$ is conjugate via the isometry $M \mathscr{F}$ to the subspace of $L^{2}\left(\mathbb{R}^{3} ; e^{4 \pi(\eta x-\tau b(x))} d x d \eta d \tau\right)$ consisting of functions that are independent of $x$. These are functions $\phi(\eta, \tau)$ supported where $\tau \geq 0$ such that $\iint|\phi(\eta, \tau)|^{2} N(2 \pi \eta, 2 \pi \tau) d \eta d \tau<+\infty$. If $\mathscr{P}_{1}$ is the orthogonal projection onto this subspace, then the Szegő projection $\mathbf{S}$ equals $\mathscr{F}^{-1} \mathcal{M}^{-1} \mathscr{P}_{1} \mathcal{M} \mathscr{F}$.

Just as in the analysis of the Bergman projection, consider the space $\widetilde{L}_{\eta, \tau}^{2}(\mathbb{R})=$ $L^{2}\left(\mathbb{R}, e^{4 \pi(\eta x-\tau b(x))} d x\right)$, and let $\mathscr{P}_{\eta, \tau}$ denote the orthogonal projection onto the subspace of constants (which is $(0)$ when $\tau<0$ ). We have the analogues of equations (6-8) and (6-9) in this situation, and the computation of the Szegő kernel $\mathscr{B}_{1}$ is carried out in exactly the same way as the computations of the Bergman kernels $\mathscr{B}_{\rho}$ for $\rho<1$. The final result is

$$
\mathscr{B}_{1}(z, w)=\frac{1}{4 \pi^{2}} \iint_{\tau \geq 0} e^{\eta\left(z_{1}+\bar{w}_{1}\right)} e^{i \tau\left(z_{2}-\bar{w}_{2}\right)} \frac{1}{N(\eta, \tau)} d \eta d \tau,
$$

and this completes the proof of part (c), and hence of Theorem 3.4.

\section{Proof of Theorem 3.6}

We estimate the kernel $\mathscr{B}_{\rho}(z, w)$ and all of its derivatives, and prove Theorem 3.6. It follows from Theorem 3.4 that

$$
\begin{aligned}
\partial_{z_{1}}^{a} \partial \bar{w}_{1} & \partial_{z_{2}}^{c} \partial \frac{d}{\bar{w}_{2}} \mathscr{M}_{\rho}(z, w) \\
& =\frac{2^{1-\rho} i^{c-d}}{4 \pi^{2}} \int_{0}^{\infty} \int_{-\infty}^{+\infty} e^{\eta\left(z_{1}+\bar{w}_{1}\right)+i \tau\left(z_{2}-\bar{w}_{2}\right)} \frac{\eta^{a+b} \tau^{c+d+1-\rho}}{\int_{-\infty}^{+\infty} e^{2(\eta r-\tau b(r))} d r} d \eta d \tau .
\end{aligned}
$$

Let

$$
A=b(x)+b(u)-2 b(\alpha) \geq 0, \quad \alpha=\frac{1}{2}(x+u), \quad \delta=h+k>0,
$$

$$
B=(t-s)+(y-v) b^{\prime}(\alpha), \quad \beta=y-v, \quad c_{\rho}=\frac{2^{1-\rho}}{4 \pi^{2} \Gamma(1-\rho)} .
$$

Then

$$
\begin{aligned}
\partial_{z_{1}}^{a} \partial_{\bar{w}_{1}}^{b} \partial_{z_{2}}^{c} \partial_{\bar{w}_{2}} \mathscr{B}_{\rho}(z, w) \\
\quad=c_{\rho} i^{c-d} \int_{0}^{\infty} \int_{-\infty}^{+\infty} e^{\eta(2 \alpha+i \beta)-\tau(b(x)+b(u)+\delta-i(t-s))} \frac{\eta^{a+b} \tau^{c+d+1-\rho}}{\int_{-\infty}^{+\infty} e^{2(\eta r-\tau b(r))} d r} d \eta d \tau \\
\quad=c_{\rho} i^{c-d} \int_{0}^{\infty} \int_{-\infty}^{+\infty} e^{i \eta \beta-\tau(A+\delta-i(t-s))} \frac{\eta^{a+b} \tau^{c+d+1-\rho}}{\int_{-\infty}^{+\infty} e^{2(\eta r-\tau(b(r+\alpha)-b(\alpha)))} d r} d \eta d \tau
\end{aligned}
$$




$$
=c_{\rho} i^{c-d} \int_{0}^{\infty} \int_{-\infty}^{+\infty} e^{-\tau(A+\delta-i B)} e^{i \eta \beta} \frac{\left(\eta+\tau b^{\prime}(\alpha)\right)^{a+b} \tau^{c+d+1-\rho}}{\int_{-\infty}^{+\infty} e^{2\left(\eta r-\tau b_{\alpha}(r)\right)} d r} d \eta d \tau .
$$

The integral in (7-2) involves two oscillatory terms: $e^{i \tau B}$ and $e^{i \eta \beta}$. We will make two estimates of the derivatives $\left|\partial_{z_{1}}^{a} \partial_{\bar{w}_{1}} \partial_{z_{2}}^{c} \partial_{\bar{w}_{2}} \mathscr{乃}_{\rho}(z, w)\right|$; one will take advantage of the oscillation if $B \neq 0$, and the other will take advantage if $\beta \neq 0$.

Case 1: $B \neq 0$. Again let $a+b+c+d+2-\rho=m$ and $a+b=n$. Making the change of variables $\eta=\tau b^{\prime}(\lambda)$, we have

$$
\begin{aligned}
&\left|\partial_{z_{1}}^{a} \partial_{\bar{w}_{1}}^{b} \partial_{z_{2}}^{c} \partial_{\bar{w}_{2}}^{d} \mathscr{P}_{\rho}(z, w)\right| \\
& \quad \leq c_{\rho} \int_{-\infty}^{+\infty}\left|\int_{0}^{\infty} \frac{\left(\eta+\tau b^{\prime}(\alpha)\right)^{a+b} \tau^{c+d+1-\rho} e^{-\tau(A+\delta-i B)}}{\int_{\mathbb{R}} e^{2\left(\eta r-\tau b_{\alpha}(r)\right)} d r} d \tau\right| d \eta \\
& \quad \leq c_{\rho} \int_{-\infty}^{+\infty}\left|\int_{0}^{\infty} \frac{b^{\prime}(\alpha+\lambda)^{n} \tau^{m} e^{-\tau(A+\delta-i B)}}{\int_{\mathbb{R}} e^{2 \tau\left(b_{\alpha}^{\prime}(\lambda) r-b_{\alpha}(r)\right)} d r} d \tau\right| b_{\alpha}^{\prime \prime}(\lambda) d \lambda \\
& \quad=c_{\rho} \int_{-\infty}^{+\infty}\left|\int_{0}^{\infty} \frac{b^{\prime}(\alpha+\lambda)^{n} \tau^{m} e^{-\tau(A+\delta-i B)} e^{-2 \tau h_{\alpha}(\lambda)}}{\varphi(\alpha+\lambda, \tau)} d \tau\right| b_{\alpha}^{\prime \prime}(\lambda) d \lambda,
\end{aligned}
$$

where in the last line we have used Proposition 4.11. According to Lemma 5.12, there is a $\sigma>0$ such that we can replace the integral in $\tau$ along the positive real axis by an integral parameterized by $\tau \rightarrow \tau(1 \pm i \sigma)$. We choose the sign so that

$$
\Re \mathrm{e}((1 \pm i \sigma)(A+\delta-i B))=A+\delta+\sigma|B| .
$$

If we make this change of contour we get

$$
\begin{aligned}
\left|\partial_{z_{1}}^{a} \partial_{\bar{w}_{1}}^{b} \partial_{z_{2}}^{c} \partial_{\bar{w}_{2}}^{d} \mathscr{P}_{\rho}(z, w)\right| & \\
& \lesssim \int_{-\infty}^{+\infty}\left|\int_{0}^{\infty} \frac{b^{\prime}(\alpha+\lambda)^{n}((1 \pm i \sigma) \tau)^{m} e^{-\tau\left[(1 \pm i \sigma)[A+\delta-i B]-2(1 \pm i \sigma) h_{\alpha}(\lambda)\right]}}{\varphi(\alpha+\lambda, \tau(1 \pm i \sigma))} d \tau\right| b_{\alpha}^{\prime \prime}(\lambda) d \lambda \\
& \lesssim \int_{-\infty}^{+\infty} \int_{0}^{\infty} \frac{\left|b^{\prime}(\alpha+\lambda)\right|^{n} \tau^{m} e^{-\tau[A+\delta+\sigma|B|]-2 \tau h_{\alpha}(\lambda)}}{\varphi(\alpha+\lambda, \tau)} b_{\alpha}^{\prime \prime}(\lambda) d \tau d \lambda \\
& =\int_{-\infty}^{+\infty} \int_{0}^{\infty} \frac{\left|b^{\prime}(\lambda)\right|^{n} \tau^{m} e^{-\tau[A+\delta+\sigma|B|]-2 \tau h_{\alpha}(\lambda-\alpha)}}{\varphi(\lambda, \tau)} b^{\prime \prime}(\lambda) d \tau d \lambda .
\end{aligned}
$$

It follows from Lemma 4.17 that this integral is bounded uniformly in $\delta \geq 0$ if $A+|B|>0$. 
Case 2: $\beta \neq 0$. Using Equation (7-2), we have

$$
\begin{aligned}
& \left|\partial_{z_{1}}^{a} \partial_{\bar{w}_{1}}^{b} \partial_{z_{2}}^{c} \partial_{\bar{w}_{2}}^{d} \Re_{\rho}(z, w)\right| \\
& \quad=c_{\rho}\left|\int_{0}^{\infty} \tau^{c+d+1-\rho} e^{-\tau(A+\delta-i B)} \int_{-\infty}^{+\infty} \frac{\left(\eta+\tau b^{\prime}(\alpha)\right)^{a+b} e^{i \eta \beta}}{\int_{\mathbb{R}} e^{2\left(\eta r-\tau b_{\alpha}(r)\right)} d r} d \eta d \tau\right| \\
& \quad \leq c_{\rho} \int_{0}^{\infty} \tau^{c+d+1-\rho} e^{-\tau(A+\delta)}\left|\int_{-\infty}^{+\infty} \frac{\left(\eta+\tau b^{\prime}(\alpha)\right)^{a+b} e^{i \eta \beta}}{\int_{\mathbb{R}} e^{2\left(\eta r-\tau b_{\alpha}(r)\right)} d r} d \eta\right| d \tau \\
& \quad=c_{\rho} \int_{0}^{\infty} \tau^{c+d+1-\rho} e^{-\tau(A+\delta)}\left|\int_{-\infty}^{+\infty}\left(\eta+\tau b^{\prime}(\alpha)\right)^{a+b} e^{i \eta \beta} N_{\alpha}(\eta, \tau)^{-1} d \eta\right| d \tau .
\end{aligned}
$$

We want to change the contour in the inner integral, replacing the integral in $\eta$ over $\mathbb{R}$ by an integral over a curve in the upper or lower half plane. According to Lemma 5.7, since $\mu$ and $\varphi$ are comparable, there is a constant $\sigma>0$ such that if $|y| \leq \sigma \varphi(\lambda+\alpha, \tau)^{-1}$, then

$$
\left|N_{\alpha}\left(\tau b_{\alpha}^{\prime}(\lambda)+i y, \tau\right)\right|=\left|\int_{-\infty}^{+\infty} e^{2\left(\tau b_{\alpha}^{\prime}(\lambda)+i y-\tau b_{\alpha}(r)\right)} d r\right|>\frac{1}{2} N_{\alpha}\left(\tau b_{\alpha}^{\prime}(\lambda), \tau\right) .
$$

Thus we can deform the integral in $\eta$ to the curve parameterized by

$$
\lambda \rightarrow z(\lambda)=\tau b_{\alpha}^{\prime}(\lambda) \pm i \sigma \varphi(\alpha+\lambda, \tau)^{-1},
$$

where we choose the sign of the imaginary part so that

$$
e^{i \eta \beta} \rightarrow e^{i z(\lambda) \beta}=\exp \left(-\frac{\sigma|\beta|}{\varphi(\alpha+\lambda, \tau)}\right) e^{i \tau b_{\alpha}^{\prime}(\lambda) \beta} .
$$

With this change of contour, $\eta+\tau b^{\prime}(\alpha)=\tau b^{\prime}(\alpha+\lambda) \pm i \sigma \varphi(\alpha+\lambda, \tau)^{-1}$, and so

$$
\left|\left(\eta+\tau b^{\prime}(\alpha)\right)\right|^{a+b} \lesssim \tau^{a+b}\left|b^{\prime}(\lambda+\alpha)\right|^{a+b}+|\sigma|^{a+b} \varphi(\alpha+\lambda, \tau)^{-a-b} .
$$

Also, $d \eta$ becomes

$$
d z=\frac{d z}{d \lambda}(\lambda) d \lambda=\left(\tau b_{\alpha}^{\prime \prime}(\lambda) \pm i \sigma \frac{\varphi^{\prime}(\alpha+\lambda, \tau)}{\varphi(\alpha+\lambda, \tau)^{2}}\right) d \lambda
$$

where $\varphi^{\prime}(\alpha+\lambda, \tau)$ means the partial derivative with respect to $\lambda$. According to the inequality in Lemma 4.14(b),

$$
\left|\tau b_{\alpha}^{\prime \prime}(\lambda) \pm i \sigma \frac{\varphi^{\prime}(\alpha+\lambda, \tau)}{\varphi(\alpha+\lambda, \tau)^{2}}\right| \leq C \tau b^{\prime \prime}(\alpha+\lambda)=C \tau b_{\alpha}^{\prime \prime}(\lambda) .
$$


Thus with this change of contour, we obtain the estimate

$$
\begin{aligned}
& \left|\partial_{z_{1}}^{a} \partial_{\bar{w}_{1}}^{b} \partial_{z_{2}}^{c} \partial_{\bar{w}_{2}}^{d} \mathscr{B}_{\rho}(z, w)\right| \\
& \lesssim \int_{0}^{\infty} \int_{-\infty}^{+\infty} e^{-\tau(A+\delta)-\sigma|\beta| / \varphi(\lambda, \tau)} \frac{\left|b^{\prime}(\lambda)\right|^{n} \tau^{m}+\sigma^{n} \varphi(\lambda, \tau)^{-n} \tau^{c+d+2-\rho}}{N_{\alpha}\left(\tau b_{\alpha}^{\prime}(\lambda-\alpha), \tau\right)} b_{\alpha}^{\prime \prime}(\lambda) d \lambda d \tau .
\end{aligned}
$$

Now $b_{\alpha}^{\prime \prime}(\lambda-\alpha)=b^{\prime \prime}(\lambda)$, and according to Proposition 5.1,

$$
N_{\alpha}\left(\tau b_{\alpha}^{\prime}(\lambda-\alpha), \tau\right)=\exp \left(2 \tau h_{\alpha}(\lambda-\alpha)\right) \varphi(\lambda, \tau) .
$$

Thus we get

$$
\begin{aligned}
\left|\partial_{z_{1}}^{a} \partial_{\bar{w}_{1}} \partial_{z_{2}}^{c} \partial_{\bar{w}_{2}}^{d} \mathscr{P}_{\rho}(z, w)\right| & \\
& \lesssim \int_{0}^{\infty} \int_{-\infty}^{+\infty} e^{-\tau(A+\delta)-\sigma|\beta| / \varphi(\lambda, \tau)-2 \tau h_{\alpha}(\lambda-\alpha)} \frac{\left|b^{\prime}(\lambda)\right|^{n} \tau^{m} b^{\prime \prime}(\lambda)}{\varphi(\lambda, \tau)} d \lambda d \tau \\
& \quad+\int_{0}^{\infty} \int_{-\infty}^{+\infty} e^{-\tau(A+\delta)-\sigma|\beta| / \varphi(\lambda, \tau)-2 \tau h_{\alpha}(\lambda-\alpha)} \frac{\tau^{c+d+2-\rho} b^{\prime \prime}(\lambda)}{\varphi(\lambda, \tau)^{n+1}} d \lambda d \tau .
\end{aligned}
$$

Theorem 3.6 now follows from Lemma 4.18.

\section{Proof of Theorem 3.8}

Let $b \in \mathscr{C}^{\infty}(\mathbb{R})$ satisfy (3-1), (3-2), and (3-4). In this section we will show that if $b$ is "sufficiently flat" at the origin, the kernel $\mathscr{B}_{\rho}(z, w)$ has singularities when $z, w \in \partial \Omega$ and $z \neq w$. Again we put

$$
\begin{aligned}
z & =\left(z_{1}, z_{2}\right)=(x+i y, t+i b(x)+i h), \quad \text { and } \\
w & =\left(w_{1}, w_{2}\right)=(u+i v, s+i b(u)+i k) .
\end{aligned}
$$

We will study the case when $x=u=0$ and $t=s$, but $y \neq v$. Then, as in (7-1), $A=B=\alpha=0, \delta=h+k>0$, and $\beta=y-v>0$. In this case, Equation (7-2) gives

$$
\begin{aligned}
& \mathscr{B}_{\rho}((i y, t+i h),(i v, t+i k)) \\
& =c_{\rho} \int_{0}^{\infty} \tau^{1-\rho} e^{-\tau(h+k)}\left(\int_{-\infty}^{+\infty} \frac{e^{i \eta(y-v)}}{\int_{\mathbb{R}} e^{2(\eta r-\tau b(r))}} d \eta\right) d r .
\end{aligned}
$$

Put

$$
I(\delta, \tau)=\int_{-\infty}^{+\infty} \frac{e^{i \eta \delta}}{\int_{\mathbb{R}} e^{2(\eta r-\tau b(r))}} d \eta=\int_{-\infty}^{+\infty} e^{i \eta \delta} N(\eta, \tau)^{-1} d \eta
$$


The function $z \rightarrow e^{i z \delta} N(z, \tau)^{-1}$ is meromorphic, and we can use Cauchy's theorem to change the contour of integration in (8-2) from $\eta \in \mathbb{R}$ to $z \in \Gamma=\Gamma_{-2}+\Gamma_{-1}+$ $\Gamma_{0}+\Gamma_{1}+\Gamma_{2}$, where

$$
\begin{aligned}
\Gamma_{-2} & =\left\{\eta \in \mathbb{R} \mid-\infty<\eta \leq-\frac{A}{\mu(\tau) \Theta(\hat{\mu}(\tau))}\right\}, \\
\Gamma_{-1} & =\left\{-\frac{A}{\mu(\tau) \Theta(\hat{\mu}(\tau))}+i y \in \mathbb{C} \mid 0 \leq y \leq \frac{3 \pi}{2 \mu(\tau)}\right\}, \\
\Gamma_{0} & =\left\{\eta+i \frac{3 \pi}{2 \mu(\tau)} \in \mathbb{C} \mid-\frac{A}{\hat{\mu}(\tau) \Theta(\hat{\mu}(\tau))} \leq \eta \leq+\frac{A}{\hat{\mu}(\tau) \Theta(\hat{\mu}(\tau))}\right\}, \\
\Gamma_{1} & =\left\{+\frac{A}{\mu(\tau) \Theta(\hat{\mu}(\tau))}+i y \in \mathbb{C} \mid \frac{3 \pi}{2 \mu(\tau)} \geq y \geq 0\right\}, \\
\Gamma_{2} & =\left\{\eta \in \mathbb{R} \mid \frac{A}{\hat{\mu}(\tau) \Theta(\hat{\mu}(\tau))} \leq \eta<+\infty\right\},
\end{aligned}
$$

since according to Corollary 5.10, $N(z, \tau) \neq 0$ when $z \in \Gamma$. Also, Corollary 5.10 shows that $N(z, \tau)^{-1}$ has one pole at $i \sigma_{\tau} / \mu(\tau)$ inside the region

$$
\left\{x+i y \in \mathbb{C} \mid-\frac{A}{\mu(\tau) \Theta(\hat{\mu}(\tau))} \leq x \leq+\frac{A}{\mu(\tau) \Theta(\hat{\mu}(\tau))}, 0 \leq y \leq \frac{3 \pi}{2 \mu(\tau)}\right\},
$$

and $\lim _{\tau \rightarrow \infty} \sigma_{\tau}=\pi$.

Proposition 8.1. Suppose that $b \in \mathscr{C}^{\infty}(\mathbb{R})$ satisfies (3-1) and (3-2), and, with $\Theta(r)$ defined as in (3-6), that $\lim _{r \rightarrow 0} \Theta(r)=0$. Then for any $|\lambda|<1$,

$$
\lim _{r \rightarrow 0} \frac{b(\lambda r)}{b(r)}=0 .
$$

Proof. Since $b^{\prime}$ is monotone increasing,

$$
\begin{aligned}
\frac{b(r)}{b(\lambda r)} & =\frac{1}{b(\lambda r)} \int_{0}^{r} b^{\prime}(s) d s=1+\frac{1}{b(\lambda r)} \int_{\lambda r}^{r} b^{\prime}(s) d s \\
& \geq 1+\frac{\lambda r b^{\prime}(\lambda r)}{b(\lambda r)} \frac{1-\lambda}{\lambda} \geq 1+\frac{1-\lambda}{\lambda} \Theta(\lambda r)^{-1},
\end{aligned}
$$

and since $\lim _{r \rightarrow 0} \Theta(\lambda r)^{-1}=+\infty$, this gives the desired result.

Proposition 8.2. Suppose again that $b \in \mathscr{C}^{\infty}(\mathbb{R})$ satisfies (3-1) and (3-2), and that $\lim _{r \rightarrow 0} \Theta(r)=0$. Let $\sigma>0$. Then for $\mu(\tau) \leq 1$,

$$
\int_{0}^{\infty} r \sin (\sigma r) e^{-2 \tau b(\hat{\mu}(\tau) r)} d r=\int_{0}^{1} r \sin (\sigma r) d r+E(\tau)
$$

where $\lim _{\hat{\mu}(\tau) \rightarrow 0^{+}} E(\tau)=0$. 
Proof. For $r \geq 1$ we have $b(\hat{\mu}(\tau) r) \geq \tau^{-1}+(r-1) \hat{\mu}(\tau) b^{\prime}(\hat{\mu}(\tau))$. Thus

$$
\begin{aligned}
\left|\int_{1}^{\infty} r \sin (\sigma r) e^{-2 \tau b(\hat{\mu}(\tau) r)} d r\right| & \leq \int_{1}^{\infty} r e^{-2-2 \tau(r-1) \hat{\mu}(\tau) b^{\prime}(\hat{\mu}(\tau))} d r \\
& =e^{-2} \int_{0}^{\infty}(r+1) e^{-2 \tau r \hat{\mu}(\tau) b^{\prime}(\hat{\mu}(\tau))} d r \\
& \lesssim\left(\tau \hat{\mu}(\tau) b^{\prime}(\hat{\mu}(\tau))\right)^{-2}+\left(\tau \hat{\mu}(\tau) b^{\prime}(\hat{\mu}(\tau))\right)^{-1} \\
& =\Theta(\hat{\mu}(\tau))^{2}+\Theta(\hat{\mu}(\tau)) .
\end{aligned}
$$

Also, it follows from Proposition 8.1 that for $0 \leq r<1$,

$$
\lim _{\hat{\mu}(\tau) \rightarrow 0} \tau b(\hat{\mu}(\tau) r)=\lim _{\hat{\mu}(\tau) \rightarrow 0} b(r \hat{\mu}(\tau)) / b(\hat{\mu}(\tau))=0 .
$$

Thus using the Lebesgue dominated convergence theorem, we have

$$
\lim _{\hat{\mu}(\tau) \rightarrow 0^{+}} \int_{0}^{1} r \sin (\sigma r)\left(e^{-2 \tau b(\hat{\mu}(\tau) r)}-1\right) d r=0 .
$$

The residue of $e^{i z \delta} N(z, \tau)^{-1}$ at $i \sigma_{\tau} / \mu(\tau)=i \sigma_{\tau} /(2 \hat{\mu}(\tau))$ is

$$
\begin{aligned}
e^{-\sigma_{\tau} \beta / \mu(\tau)} N^{\prime}\left(i \sigma_{\tau} / \mu(\tau), \tau\right)^{-1} & \\
& =\frac{1}{2} e^{-\sigma_{\tau} \beta / \mu(\tau)}\left(\int_{-\infty}^{+\infty} r e^{2 i r \sigma_{\tau} / \mu(\tau)} e^{-2 \tau b(r)} d r\right)^{-1} \\
& =\frac{2}{\mu(\tau)^{2}} e^{-\sigma_{\tau} \beta / \mu(\tau)}\left(\int_{-\infty}^{+\infty} r e^{i r \sigma_{\tau}} e^{-2 \tau b(\hat{\mu}(\tau) r)} d r\right)^{-1} \\
& =\frac{1}{i \mu(\tau)^{2}} e^{-\sigma_{\tau} \beta / \mu(\tau)}\left(\int_{0}^{+\infty} r \sin \left(r \sigma_{\tau}\right) e^{-2 \tau b(\hat{\mu}(\tau) r)} d r\right)^{-1} \\
& =\frac{1}{i \mu(\tau)^{2}} e^{-\sigma_{\tau} \beta / \mu(\tau)}\left(\int_{0}^{1} r \sin \left(r \sigma_{\tau}\right) d r+E(\tau)\right)^{-1},
\end{aligned}
$$

where $E(\tau) \rightarrow 0$ as $\mu(\tau) \rightarrow 0$ by Proposition 8.2. Moreover, since $\sigma_{\tau} \rightarrow \pi$, it follows that

$$
I(\beta, \tau)=\frac{\pi^{2}}{\mu(\tau)^{2}} e^{-\pi \beta / \mu(\tau)}(1+o(1))+\int_{\Gamma} e^{i \beta z} N(z, \tau)^{-1} d z
$$

We show that the last integral is smaller than the main term. Note that

$$
\mu(\tau) \Theta(\hat{\mu}(\tau)) \approx \frac{b(\mu(\tau))}{b^{\prime}(\mu(\tau))}=\frac{1}{\tau b^{\prime}(\hat{\mu}(\tau))},
$$

so

$$
\frac{A}{\mu(\tau) \Theta(\hat{\mu}(\tau))} \approx B \tau b^{\prime}(\mu(\tau)) .
$$


Proposition 8.3. Let $B>0$. Then there are constants $C$ and $c$ depending only on B such that

$$
\int_{B \tau b^{\prime}(\mu(\tau))}^{\infty} N(\eta, \tau)^{-1} d \eta \leq C \frac{1}{\mu(\tau)^{2}} e^{-c / \Theta(\hat{\mu}(\tau))}
$$

Proof. Recall that $N\left(\tau b^{\prime}(\lambda), \tau\right)=e^{2 \tau h(\lambda)} \varphi(\lambda, \tau)$. Thus if we make the change of variables $\eta=\tau b^{\prime}(\lambda)$

$$
\begin{aligned}
\int_{B \tau b^{\prime}(\hat{\mu}(\tau))}^{\infty} N(\eta, \tau)^{-1} d \eta & =\int_{\lambda_{0}}^{\infty} N\left(\tau b^{\prime}(\lambda), \tau\right)^{-1} \tau b^{\prime \prime}(\lambda) d \lambda \\
& =\int_{\lambda_{0}}^{\infty} e^{-2 \tau h(\lambda)} \frac{1}{\varphi(\lambda, \tau)} \tau b^{\prime \prime}(\lambda) d \lambda
\end{aligned}
$$

where $b^{\prime}\left(\lambda_{0}\right)=B b^{\prime}(\hat{\mu}(\tau))$. By Lemma 4.14, $\varphi(\lambda, \tau)^{-1} \leq \varphi(0, \tau)^{-1}+A \tau b^{\prime}(\lambda)$, with $A$ an absolute constant. Thus since

$$
h^{\prime}(\lambda)=\lambda b^{\prime \prime}(\lambda) \quad \text { and } \quad \lambda^{-1} h(\lambda) \leq b^{\prime}(\lambda) \leq \lambda^{-1}(h(\lambda)+b(\lambda)) \leq 2 \lambda^{-1} h(\lambda)
$$

we have

$$
\begin{aligned}
\int_{B \tau b^{\prime}(\mu(\tau))}^{\infty} N(\eta, \tau)^{-1} d \eta & \leq \frac{1}{\lambda_{0}} \int_{\lambda_{0}}^{\infty} e^{-2 \tau h(\lambda)}\left((\varphi(0, \tau))^{-1}+A \tau b^{\prime}(\lambda)\right) \tau \lambda b^{\prime \prime}(\lambda) \\
& \leq \frac{1}{\lambda_{0} \varphi(0, \tau)} e^{-2 \tau h\left(\lambda_{0}\right)}+\frac{A}{\lambda_{0}^{2}} \tau h\left(\lambda_{0}\right) e^{-2 \tau h\left(\lambda_{0}\right)} \\
& \leq \frac{1}{\lambda_{0} \varphi(0, \tau)} e^{-\tau h\left(\lambda_{0}\right)} \frac{A}{\lambda_{0}^{2}} \tau h\left(\lambda_{0}\right) e^{-\tau h\left(\lambda_{0}\right)}
\end{aligned}
$$

But now we observe that $\lambda_{0} \approx \mu(\tau)$. Since $b^{\prime \prime}(r)$ is monotone increasing for $r>0$, we know that $b^{\prime}(2 r) \geq 2 b^{\prime}(r)$, and so for any integer $m \geq 1$ we have $2^{m} b^{\prime}\left(2^{-m} r\right) \leq b^{\prime}(r) \leq 2^{-m} b^{\prime}\left(2^{m} r\right)$. Now if $B \leq 1$, then $b^{\prime}\left(\lambda_{0}\right) \leq b^{\prime}(\mu(\tau))$, so $\lambda_{0} \leq \mu(\tau)$. Let $m$ be the nonnegative integer such that $2^{-m-1} \leq B<2^{-m}$. Then $b^{\prime}\left(\lambda_{0}\right) \geq 2^{-m-1} b^{\prime}(\mu(\tau)) \geq b^{\prime}\left(2^{-m-1} \mu(\tau)\right)$, and so $\lambda_{0} \geq 2^{-m-1} \mu(\tau) \geq B \mu(\tau) / 2$. Thus we have $B \mu(\tau) / 2 \leq \lambda_{0} \leq \mu(\tau)$.

On the other hand, if $B \geq 1$, then $b^{\prime}\left(\lambda_{0}\right) \geq b^{\prime}(\mu(\tau))$ so $\lambda_{0} \geq \mu(\tau)$. Let $m$ be the nonnegative integer such that $2^{m} \leq B<2^{m+1}$. Then $b^{\prime}\left(\lambda_{0}\right) \leq 2^{m+1} b^{\prime}(\mu(\tau)) \leq$ $b^{\prime}\left(2^{m+1} \mu(\tau)\right)$, and so $\lambda_{0} \leq 2^{m+1} \mu(\tau) \leq 2 B \mu(\tau)$. Thus $\mu(\tau) \leq \lambda_{0} \leq 2 B \mu(\tau)$.

Now for any $r$,

$$
\frac{b(r)}{b^{\prime}(r)} \leq r \Theta(r) \leq C \frac{b(r)}{b^{\prime}(r)}
$$


Thus

$$
\begin{aligned}
\tau h\left(\lambda_{0}\right) & \approx \tau \lambda_{0} b^{\prime}\left(\lambda_{0}\right)=B \tau \lambda_{0} b^{\prime}(\mu(\tau)) \\
& =B \tau \lambda_{0} \frac{b^{\prime}(\mu(\tau))}{b(\mu(\tau))} b(\mu(\tau))=B \frac{\lambda_{0}}{\mu(\tau) \Theta(\mu(\tau))} \approx \frac{1}{\Theta(\mu(\tau))},
\end{aligned}
$$

where $\tau h\left(\lambda_{0}\right) \approx(\Theta(\mu(\tau)))^{-1}$ means that the ratio is bounded above and below by a constant that depends on $B$. This completes the proof.

We now estimate the integral over $\Gamma$. First, by Proposition 8.3, we have

$$
\left|\int_{\Gamma_{ \pm 2}} e^{i \beta z} N(z, \tau)^{-1} d z\right| \leq \int_{A /(\mu(\tau) \Theta(\hat{\mu}(\tau)))}^{\infty} N(\eta, \tau)^{-1} d \eta \leq \frac{A}{\mu(\tau)^{2}} e^{-c / \Theta(\mu(\tau))} .
$$

Next, if we put $S(z, \tau)=z^{-1} \sinh (\mu(\tau) z)$, then we know from Theorem 5.9 that $|N(z, \tau)-S(z, \tau)| \leq|S(z, \tau)| / 2$ on $\Gamma_{ \pm 1}$. It follows that on $\Gamma_{ \pm 1}$, we have $|N(z, \tau)|^{-1} \leq 2|S(z, \tau)|^{-1}$. Thus

$$
\left|\int_{\Gamma_{ \pm 1}} e^{i \beta z} N(z, \tau)^{-1} d z\right| \leq \int_{\Gamma_{1}}|N(z, \tau)|^{-1} d|z| \leq 2 \int_{\Gamma_{1}}|S(z, \tau)|^{-1} d|z| .
$$

If $z \in \Gamma_{1}$, then $z=A /(\mu(\tau) \Theta(\hat{\mu}(\tau)))+i y$ with $0 \leq y \leq 3 \pi /(2 \mu(\tau))$. Thus for such $z$,

$$
\begin{aligned}
|S(z, \tau)|^{-1} & =\left|\frac{A}{\mu(\tau) \Theta(\hat{\mu}(\tau))}+i y\right| \frac{1}{|\sinh (A / \Theta(\hat{\mu}(\tau))+i \mu(\tau) y)|} \\
& =\left|\frac{A}{\mu(\tau) \Theta(\hat{\mu}(\tau))}+i y\right| \frac{1}{\sqrt{\sinh ^{2}(A / \Theta(\hat{\mu}(\tau)))+\sin ^{2}(\mu(\tau) y)}} \\
& \leq\left|\frac{A}{\mu(\tau) \Theta(\hat{\mu}(\tau))}+i y\right| \frac{1}{|\sinh (2 A / \Theta(\hat{\mu}(\tau)))|} \\
& \leq A\left(\frac{1}{\mu(\tau) \Theta(\hat{\mu}(\tau))}+\frac{1}{\mu(\tau)}\right) e^{-c / \Theta(\hat{\mu}(\tau))} .
\end{aligned}
$$

If $\Theta(\hat{\mu}(\tau))$ is small enough and we estimate $\Theta(\hat{\mu}(\tau))^{-1} \exp \left(-c \Theta(\hat{\mu}(\tau))^{-1}\right)$ by $A \exp \left(-c^{\prime} \Theta(\hat{\mu}(\tau))^{-1}\right)$ where $c^{\prime}<c$, it follows that

$$
\left|\int_{\Gamma_{ \pm 1}} e^{i \beta z} N(z, \tau)^{-1} d z\right| \leq \frac{A}{\mu(\tau)^{2}} e^{-c / \Theta(\mu(\tau))} .
$$

Finally if $z \in \Gamma_{0}$, then $i \beta z=i \beta s-3 \pi \beta /(2 \mu(\tau))$, so

$$
\begin{aligned}
\left|\int_{\Gamma_{0}} e^{i \beta z} N(z, \tau)^{-1} d z\right| & \leq \int_{|s|<A /(\mu(\tau) \Theta(\hat{\mu}(\tau)))} e^{-3 \pi \beta /(2 \mu(\tau))}\left|N\left(s+\frac{3 \pi i}{2 \mu(\tau)}, \tau\right)\right|^{-1} d s \\
& \leq 2 e^{-3 \pi \beta /(2 \mu(\tau))} \int_{|s|<A /(\mu(\tau) \Theta(\hat{\mu}(\tau)))}\left|S\left(s+\frac{3 \pi i}{2 \mu(\tau)}, \tau\right)\right|^{-1} d s .
\end{aligned}
$$


But since $\sinh ^{2}(x)+1=(\cosh (2 x)+1) / 2>e^{2 x} / 2$, we have

$$
\begin{aligned}
\left|S\left(s+\frac{3 \pi i}{2 \mu(\tau)}, \tau\right)\right|^{-1} & \leq\left(|s|+\frac{3 \pi}{2 \mu(\tau)}\right) \frac{1}{\sqrt{\sinh ^{2}(\mu(\tau) s)+\sin ^{2}(3 \pi / 2)}} \\
& =\left(|s|+\frac{3 \pi}{2 \mu(\tau)}\right) \frac{1}{\sqrt{\sinh ^{2}(\mu(\tau) s)+1}} \\
& \leq\left(|s|+\frac{3 \pi}{2 \mu(\tau)}\right) e^{-2 \mu(\tau) s},
\end{aligned}
$$

and so

$|s|<A /(\mu(\tau) \Theta(\hat{\mu}(\tau)))$

$$
\left|S\left(s+\frac{3 \pi i}{2 \mu(\tau)}, \tau\right)\right|^{-1} d s \leq A \int_{0}^{\infty}\left(s+\frac{1}{\mu(\tau)}\right) e^{-2 \mu(\tau) s} d s \leq \frac{A}{\mu(\tau)^{2}} .
$$

Thus

$$
\left|\int_{\Gamma_{0}} e^{i \beta z} N(z, \tau)^{-1} d z\right| \leq \frac{A}{\mu(\tau)^{2}} \exp \left(-\frac{3 \pi \beta}{2 \mu(\tau)}\right)
$$

It now follows from equations $(8-1),(8-2),(8-4),(8-8),(8-9)$, and $(8-10)$ that for $\mu(\tau)$ sufficiently small we have

$$
\mathscr{B}_{\rho}((i y, t+i h),(i v, t+i k)) \geq c \int_{0}^{\infty} \tau^{1-\rho} e^{-\tau \delta} e^{-\pi \beta / \mu(\tau)} \mu(\tau)^{-2} d \tau .
$$

If we make the change of variables $r=\mu(\tau)$, then $b(r)=\tau^{-1}$, and so $d \tau=$ $-b(r)^{-2} b^{\prime}(r) d r$. Thus we need to consider the integral

$$
H(\delta, \beta)=\int_{0}^{\infty} \exp \left(-\frac{\delta}{b(r)}-\frac{\pi \beta}{r}\right) \frac{b^{\prime}(r)}{b(r)^{3-\rho}} \frac{d r}{r^{2}} .
$$

However, if $b$ is supercritical, $\lim _{\delta \rightarrow 0} H(\delta, \beta)=+\infty$.

\section{Appendix: The $\mathscr{H}^{p}(\Omega)$ spaces}

In this appendix, we use the ideas in [Stein 1993] to develop the theory of the Hardy spaces $\mathscr{H}^{p}(\Omega)$ for model unbounded domains. For simplicity, we restrict attention to $1<p<\infty$, although the results are also true if $p=1$ or $p=\infty$.

\section{A.1. Definitions and statement of results.}

Let $\varphi: \mathbb{C}^{n} \rightarrow \mathbb{R}$ be upper semicontinuous, and put

$$
\Omega_{\varphi}=\Omega=\left\{\left(z, z_{n+1}\right) \in \mathbb{C}^{n+1} \mid \Im \mathrm{m}\left(z_{n+1}\right)>\varphi(z)\right\} .
$$

Identify the boundary $\partial \Omega$ with $\mathbb{C}^{n} \times \mathbb{R}$ so that a point $(z, x) \in \mathbb{C}^{n} \times \mathbb{R}$ corresponds to $(z, x+i \varphi(z)) \in \partial \Omega$. Let $d z d x$ denote Lebesgue measure on $\mathbb{C}^{n} \times \mathbb{R}$. Then integration on the boundary of $\Omega$ means integration with respect to $d z d x$. 
Notation A.2. $\quad$ For each $z \in \mathbb{C}^{n}$ and each $\epsilon>0$, let

$$
r(z, \epsilon)=\sup \{r \geq 0|| z-w \mid \leq r \text { implies } \varphi(w)<\varphi(z)+\epsilon\} .
$$

Since $\varphi$ is upper semicontinuous, it follows that $r(z, \epsilon)>0$.

- Let $F: \Omega \rightarrow \mathbb{C}$. For $\epsilon>0$, put $F_{\epsilon}\left(z, z_{n+1}\right)=F\left(z, z_{n+1}+i \epsilon\right) . F_{\epsilon}$ is defined in a neighborhood of the closure $\bar{\Omega}$ of $\Omega$. We use the same notation to denote the restriction $F_{\epsilon}: \partial \Omega \rightarrow \mathbb{C}$ of this function to the boundary.

- The upper half-plane in $\mathbb{C}$ is $U=\{x+i y \in \mathbb{C} \mid y>0\}$.

- Let $F: \Omega \rightarrow \mathbb{C}$. For $z \in \mathbb{C}^{n}$ and $\epsilon \geq 0$, put $f_{z, \epsilon}(x+i y)=F(z, x+i y+i \varphi(z)+i \epsilon)$. Then $f_{z, \epsilon}: \mathcal{U} \rightarrow \mathbb{C}$.

- Let $F: \Omega \rightarrow \mathbb{C}$. The "radial" maximal function is

$$
\mathcal{N}_{0}[F](z, x)=\sup _{y>0}|F(z, x+i \varphi(z)+i y)| .
$$

Remark A.3. If $F: \Omega \rightarrow \mathbb{C}$ is holomorphic, then $F_{\epsilon}$ is holomorphic on a neighborhood of $\bar{\Omega}$ if $\epsilon>0$, and $f_{z, \epsilon}$ is holomorphic on a neighborhood of $\bar{u}$ if $z \in \mathbb{C}^{n}$ and $\epsilon>0$.

Definition A.4. For $1<p<+\infty, \mathscr{H}^{p}(\Omega)$ is the space of holomorphic functions $F$ on $\Omega$ such that

$$
\|F\|_{\mathscr{H} p(\Omega)}^{p}=\sup _{\epsilon>0}\left\|F_{\epsilon}\right\|_{L^{p}(\partial \Omega)}^{p}=\sup _{\epsilon>0} \iint_{\mathbb{C}^{n} \times \mathbb{R}}|F(z, x+i \varphi(z)+i \epsilon)|^{p} d z d x<+\infty .
$$

Our objective is to prove the following:

Lemma A.5. Let $F \in \mathscr{H}^{p}(\Omega)$. Then there exists $F^{b} \in L^{p}(\partial \Omega)$ with the following properties.

(a) For almost every $(z, x) \in \mathbb{C}^{n} \times \mathbb{R}$,

$$
\lim _{y \rightarrow 0^{+}} F(z, x+i \varphi(z)+i y)=F^{b}(z, x+i \varphi(z)) .
$$

(b) $\left\|F^{b}\right\|_{L^{p}(\partial \Omega)}=\|F\|_{\mathscr{H}^{p}(\Omega)}$.

(c) $\lim _{\epsilon \rightarrow 0^{+}}\left\|F_{\epsilon}-F^{b}\right\|_{L^{p}(\partial \Omega)}=0$.

(d) There is a constant $C_{0}$ independent of $F$ with $\left\|\mathcal{N}_{0}[F]\right\|_{L^{p}(\partial \Omega)} \leq C_{0}\|F\|_{\mathscr{H}(\Omega)}$.

(e) The boundary function $F^{b}$ is annihilated (in the sense of distributions) by all tangential Cauchy-Riemann operators on $\partial \Omega$. 
A.6. The one-variable case. As in [Stein 1993], we reduce proving Lemma A.5 to a similar statement in one variable. We first recall the definition and some basic results about the classical Hardy spaces on the upper half-plane $U$.

Definition A.7. For $1<p<+\infty$, denote by $\mathscr{H}^{p}(U)$ the space of holomorphic functions $F$ on $U$ such that

$$
\|F\|_{\mathscr{H} p(U)}^{p}=\sup _{y>0} \int_{\mathbb{R}}|F(x+i y)|^{p} d x<+\infty .
$$

We use the same notation as in the several-variable case. If $F$ is a complex-valued function defined on $\mathcal{U}$, the radial maximal function is

$$
\mathcal{N}_{0}[F](x)=\sup _{y>0}|F(x+i y)| .
$$

For $F \in \mathscr{H}^{p}(U)$ and $\epsilon>0$, set

$$
F_{\epsilon}(x+i y)=F(x+i y+i \epsilon) .
$$

$F_{\epsilon}$ is defined in a neighborhood of $\overline{\mathcal{U}}$, and when restricted to the boundary $\mathbb{R}$, we have $F_{\epsilon} \in L^{p}(\mathbb{R})$, and $\|F\|_{\mathscr{C}^{p}(\mathscr{U})}=\sup _{\epsilon>0}\left\|F_{\epsilon}\right\|_{L^{p}(\mathbb{R})}$.

The following result describes some of the basic properties of the spaces $\mathscr{H}^{p}(\mathcal{U})$. A proof of the more general statements for harmonic functions can be found in [Stein and Weiss 1971].

Lemma A.8. Let $1<p<\infty$, and let $F \in \mathscr{H}^{p}(\mathcal{U})$.

(a) There exists $F^{b} \in L^{p}(\mathbb{R})$ such that $\lim _{\epsilon \rightarrow 0^{+}}\left\|F^{b}-F_{\epsilon}\right\|_{L^{p}(\mathbb{R})}=0$.

(b) There is a constant $C_{0}$ independent of $F$ with $\left\|\mathcal{N}_{0}[F]\right\|_{L^{p}(\mathbb{R})} \leq C_{0}\|F\|_{\mathscr{H}^{p}(U)}$.

(c) There exists a subset $E \subset \mathbb{R}$ of Lebesgue measure zero such that for $x \notin E$, $\lim _{y \rightarrow 0^{+}} F(x+i y)=F^{b}(x)$.

(d) $\|F\|_{\mathscr{H}^{p}(U)}=\left\|F^{b}\right\|_{L^{p}(\mathbb{R})}$.

(e) If $z=x+i y \in \mathcal{U}$, then

$$
F(z)=\frac{1}{2 \pi i} \int_{\mathbb{R}} \frac{F^{b}(t)}{t-z} d t=\frac{1}{\pi} \int_{\mathbb{R}} \frac{y}{y^{2}+(t-x)^{2}} F^{b}(t) d t .
$$

A.9. Proof of Lemma A.5. The key fact that allows us to reduce the situation in several variables to the one-variable case is the following.

Proposition A.10. Let $1<p<\infty$, and let $F \in \mathscr{H}^{p}(\Omega)$. For every $z \in \mathbb{C}^{n}$ and for every $\epsilon>0$, the function $f_{z, \epsilon} \in \mathscr{H}^{p}(U)$, and

$$
\left\|f_{z, \epsilon}\right\|_{\mathscr{H}^{p}(U)} \leq 4^{1 / p}\left(\pi \omega_{n} r(z, \epsilon)^{2 n}\right)^{-1 / p}\|F\|_{\mathscr{H}^{p}(\Omega)},
$$

where $\omega_{n}$ is the volume of the unit ball in $\mathbb{C}^{n}$. 
Proof. Let $z \in \mathbb{C}^{n}, \epsilon>0$, and $x+i y \in U$. Let $\chi$ be the characteristic function of the set

$$
\triangle=\left\{\left(w, w_{n+1}\right) \in \mathbb{C}^{n+1}|| w \mid<r(z, \epsilon) \text { and }\left|w_{n+1}\right|<y\right\},
$$

which is the Cartesian product of a ball of radius $r(z, \epsilon)$ in $\mathbb{C}^{n}$ and a disk of radius $y$ in $\mathbb{C}$. It follows from $(\mathrm{A}-1)$ that if $\chi(z-w,(x+i y)-(u+i v)) \neq 0$, then $\varphi(z)>\varphi(w)-\epsilon$ and $v>0$, so $v+\varphi(z)+\epsilon>\varphi(w)$. In particular, the inequality $\chi(z-w, x-u+i(y-v)) \neq 0$ implies $(w, u+i v+i \varphi(z)+i \epsilon) \in \Omega$. The volume of $\triangle$ is $\pi \omega_{n} r(z, \epsilon)^{2 n} y^{2}$. It follows from the plurisubharmonicity (that is, the submean-value property) of $\left|F_{\epsilon}\right|^{p}$ that

$$
\begin{aligned}
\pi \omega_{n} r & (z, \epsilon)^{2 n} y^{2}\left|f_{z, \epsilon}(x+i y)\right|^{p} \\
& =\pi \omega_{n} r(z, \epsilon)^{2 n} y^{2}|F(z, x+i y+i \varphi(z)+i \epsilon)|^{p} \\
& \leq \int_{\mathbb{C}^{n+1}} \chi(z-w, x-u+i(y-v))|F(w, u+i v+i \varphi(z)+i \epsilon)|^{p} d w d u d v \\
& =\int_{\mathbb{C}^{n+1}} \chi(z-w, u+i(y-v))|F(w, x-u+i v+i \varphi(z)+i \epsilon)|^{p} d w d u d v
\end{aligned}
$$

Integrating with respect to $x \in \mathbb{R}$ then gives

$$
\begin{aligned}
& \pi \omega_{n} r(z, \epsilon)^{2 n} y^{2} \int_{\mathbb{R}}\left|f_{z, \epsilon}(x+i y)\right|^{p} d x \\
& \leq \int_{\mathbb{C}^{n+1}} \chi(z-w, u+i(y-v)) \int_{x \in \mathbb{R}}|F(w, x-u+i v+i \varphi(z)+i \epsilon)|^{p} d x d w d u d v \\
& \quad \leq \int_{\substack{|y-v|<y \\
|u|<y}} \int_{w \in \mathbb{C}^{n}} \int_{x \in \mathbb{R}}|F(w, x+i v+i \varphi(z)+i \epsilon)|^{p} d x d w d u d v \leq 4 y^{2}\|F\|_{\mathscr{H} p}^{p}(\Omega) .
\end{aligned}
$$

Taking the supremum in $y$ gives $\left\|f_{z, \epsilon}\right\|_{\mathscr{H}^{p}(\vartheta)}^{p} \leq 4\left(\pi \omega_{n} r(z, \epsilon)^{2 n}\right)^{-1}\|F\|_{\mathscr{H}^{p}(\Omega)}^{p}$, as claimed.

Recall that if $F \in \mathscr{H}^{p}(\Omega)$, then

$$
\mathcal{N}_{0}[F](z, x+i \varphi(z))=\sup _{y>0}|F(z, x+i \varphi(z)+i y)| .
$$

We then can now prove Lemma A.5(d), the analogue of Lemma A.8(b).

Corollary A.11. Let $1<p<\infty$, and let $F \in \mathscr{H}^{p}(\Omega)$. Then

$$
\int_{\mathbb{C}^{n} \times \mathbb{R}}\left|\mathcal{N}_{0}[F](z, x+i \varphi(z))\right|^{p} d z d x \leq C_{0}^{p}\|F\|_{\mathscr{F}^{p}(\Omega)}^{p} .
$$


Proof. Let $z \in \mathbb{C}^{n}$ and $\epsilon>0$. Since $f_{z, \epsilon} \in \mathscr{H}^{p}(\mathcal{U})$, it follows from Lemma A.8(b) that

$$
\int_{x \in \mathbb{R}} \sup _{y>0}|F(z, x+i y+i \epsilon+i \varphi(z))|^{p} d x \leq C_{0}^{p} \int_{x \in \mathbb{R}}|F(z, x+i \epsilon+i \varphi(z))|^{p} d x .
$$

Integrating with respect to $z \in \mathbb{C}^{n}$ then gives

$$
\begin{aligned}
\int_{\mathbb{C}^{n} \times \mathbb{R}} \sup _{y>0}|F(z, x+i y+i \epsilon+i \varphi(z))|^{p} d z d x & \leq C_{0}^{p} \int_{\mathbb{C}^{n} \times \mathbb{R}}|F(z, x+i \epsilon+i \varphi(z))|^{p} d z d x \\
& \leq C_{0}^{p}\|F\|_{\mathscr{H}^{p}(\Omega)}^{p} .
\end{aligned}
$$

Since the right hand side is independent of $\epsilon$, we can let $\epsilon \rightarrow 0$ and obtain

$$
\iint_{\mathbb{C}^{n} \times \mathbb{R}} \sup _{y>0}|F(z, x+i \varphi(z)+i y)|^{p} d z d x \leq C_{0}^{p}\|F\|_{\mathscr{H} p}^{p}(\Omega) .
$$

We turn to the proof of the other parts of Lemma A.5. It follows from the inequality in Corollary A.11 that if $F \in \mathscr{H}^{p}(\Omega)$, then for almost every $z \in \mathbb{C}^{n}$, the function $f_{z}(x+i y)=F(z, x+i \varphi(z)+i y)$ belongs to $\mathscr{H}^{p}(u)$. Let $E \subseteq \mathbb{C}^{n}$ be the set where $f_{z} \notin_{\mathscr{H}^{p}}(U)$, so $|E|=0$. Then for each $z \notin E$, we can apply Lemma A.8 to the function $f_{z}$. In particular, since functions in $\mathscr{H}^{p}(U)$ have boundary values, the limit

$$
F^{b}(z, x+i \varphi(z))=\lim _{y \rightarrow 0^{+}} F(z, x+i \varphi(z)+i y)
$$

exists for almost every $x \in \mathbb{R}$ if $z \notin E$. In particular, $F^{b}(z, x+i \varphi(z))$ exists for almost every $(z, x+i \varphi(z)) \in \partial \Omega$. Moreover, for $z \notin E$,

$$
\lim _{y \rightarrow 0^{+}} \int_{\mathbb{R}}\left|F(z, x+i \varphi(z)+i y)-F^{b}(z, x+i \varphi(z))\right|^{p} d x=0,
$$

and

$$
\int_{\mathbb{R} y>0} \sup _{y>0}|F(z, x+i \varphi(z)+i y)|^{p} \leq C_{0}^{p} \int_{\mathbb{R}}\left|F^{b}(z, x+i \varphi(z))\right|^{p} d x .
$$

Proposition A.12. Let $1<p<\infty$, and let $F \in \mathscr{H}^{p}(\Omega)$.

(a) $\lim _{\epsilon \rightarrow 0^{+}}\left\|F^{b}-F_{\epsilon}\right\|_{L^{p}(\partial \Omega)}=0$.

(b) $\left\|F^{b}\right\|_{L^{p}(\partial \Omega)}=\|F\|_{\mathscr{H}^{p}(\Omega)}$. 
Proof. It follows from (A-4) that $\lim _{\epsilon \rightarrow 0^{+}} F_{\epsilon}(z, x)=F^{b}(z, x)$ for almost every $(z, x) \in \partial \Omega$. Also, we have $\sup _{\epsilon>0}\left|F_{\epsilon}(z, x)\right|=\mathcal{N}_{0}[F](z, x)$, and by Corollary A.11, $\mathcal{N}_{0}[F] \in L^{p}(\partial \Omega)$. Thus (a) follows from the Lebesgue dominated convergence theorem.

To establish (b), first note that it follows from Fatou's lemma that

$$
\begin{aligned}
\iint_{\mathbb{C}^{n} \times \mathbb{R}}\left|F^{b}(z, x+i \varphi(z))\right|^{p} d z d x & =\iint_{\mathbb{C}^{n} \times \mathbb{R}} \lim _{\epsilon \rightarrow 0^{+}}\left|F^{b}(z, x+i \varphi(z)+i \epsilon)\right|^{p} d z d x \\
& \leq \limsup _{\epsilon \rightarrow 0^{+}} \iint_{\mathbb{C}^{n} \times \mathbb{R}}\left|F^{b}(z, x+i \varphi(z)+i \epsilon)\right|^{p} d z d x \\
& \leq\|F\|_{\mathscr{H} p(\Omega)}^{p} .
\end{aligned}
$$

On the other hand, if $z \notin E$ so that $f_{z} \in \mathscr{H}^{p}(\mathcal{U})$,

$$
\begin{aligned}
\int_{\mathbb{R}}\left|F^{b}(z, x+i \varphi(z))\right|^{p} d x & =\int_{\mathbb{R}}\left|f_{z}^{b}(x)\right|^{p} d x=\sup _{\epsilon>0} \int_{\mathbb{R}}\left|f_{z}(x+i \epsilon)\right|^{p} d x \\
& =\sup _{\epsilon>0} \int_{\mathbb{R}}|F(z, x+i \varphi(z)+i \epsilon)|^{p} d x .
\end{aligned}
$$

Since this holds for almost every $z \in \mathbb{C}^{n}$, integrating with respect to $z \in \mathbb{C}^{n}$ gives

$$
\begin{aligned}
\iint_{\mathbb{C}^{n} \times \mathbb{R}}|F(z, x+i \varphi(z)+i \epsilon)|^{p} d z d x & \leq \int_{\mathbb{C}^{n}}\left(\sup _{\epsilon>0} \int_{\mathbb{R}}|F(z, x+i \varphi(z)+i \epsilon)|^{p} d x\right) d z \\
& =\int_{\mathbb{C}^{n}}\left(\int_{\mathbb{R}}\left|F^{b}(z, x+i \varphi(z))\right|^{p} d x\right) d z \\
& =\iint_{\mathbb{C}^{n} \times \mathbb{R}}\left|F^{b}(z, x+i \varphi(z))\right|^{p} d z d x .
\end{aligned}
$$

This shows that $\left\|F^{b}\right\|_{L^{p}(\partial \Omega)}=\|F\|_{\mathscr{H}^{p}(\Omega)}$, and completes the proof.

\section{Acknowledgment}

We thank the referee for providing us with several of the references we have cited.

\section{References}

[Bell 1986] S. Bell, "Differentiability of the Bergman kernel and pseudolocal estimates", Math. Z. 192:3 (1986), 467-472. MR 87i:32034 Zbl 0594.32025

[Boas 1987] H. P. Boas, "Extension of Kerzman's theorem on differentiability of the Bergman kernel function”, Indiana Univ. Math. J. 36:3 (1987), 495-499. MR 88j:32028 Zbl 0628.32030

[Boas et al. 1995] H. P. Boas, E. J. Straube, and J. Y. Yu, "Boundary limits of the Bergman kernel and metric", Michigan Math. J. 42:3 (1995), 449-461. MR 96j:32029 Zbl 0853.32028

[Bruna et al. 1988] J. Bruna, A. Nagel, and S. Wainger, "Convex hypersurfaces and Fourier transforms", Ann. of Math. (2) 127:2 (1988), 333-365. MR 89d:42023 Zbl 0666.42010 
[Catlin 1987] D. Catlin, "Subelliptic estimates for the $\bar{\partial}$-Neumann problem on pseudoconvex domains”, Ann. of Math. (2) 126:1 (1987), 131-191. MR 88i:32025 Zbl 0627.32013

[Christ 2001a] M. Christ, "Hypoellipticity in the infinitely degenerate regime", pp. 59-84 in Complex analysis and geometry (Columbus, OH, 1999), edited by J. D. McNeal, Ohio State Univ. Math. Res. Inst. Publ. 9, de Gruyter, Berlin, 2001. MR 2003e:35044 Zbl 1015.32032

[Christ 2001b] M. Christ, "Spiraling and nonhypoellipticity", pp. 85-101 in Complex analysis and geometry (Columbus, OH, 1999), edited by J. D. McNeal, Ohio State Univ. Math. Res. Inst. Publ. 9, de Gruyter, Berlin, 2001. MR 2003g:32053 Zbl 1014.32032

[Christ 2002] M. Christ, "Hypoellipticity of the Kohn Laplacian for three-dimensional tubular Cauchy-Riemann structures", J. Inst. Math. Jussieu 1:2 (2002), 279-291. MR 2003k:32048 Zbl 1038.32031

[Christ and Geller 1992] M. Christ and D. Geller, "Counterexamples to analytic hypoellipticity for domains of finite type", Ann. of Math. (2) 135:3 (1992), 551-566. MR 93i:35034 Zbl 0758.35024

[Diederich and Fornaess 1978] K. Diederich and J. E. Fornaess, "Pseudoconvex domains with realanalytic boundary”, Ann. Math. (2) 107:2 (1978), 371-384. MR 57 \#16696 Zbl 0378.32014

[Fu 1994] S. Fu, "A sharp estimate on the Bergman kernel of a pseudoconvex domain", Proc. Amer. Math. Soc. 121:3 (1994), 979-980. MR 94m:32035 Zbl 0808.32024

[Haslinger 1995] F. Haslinger, "Singularities of the Szegő kernel for certain weakly pseudoconvex domains in $\mathbb{C}^{2}$,, J. Funct. Anal. 129:2 (1995), 406-427. MR 96g:32045 Zbl 0853.32023

[Haslinger 1998] F. Haslinger, "Bergman and Hardy spaces on model domains", Illinois J. Math. 42:3 (1998), 458-469. MR 99f:32004 Zbl 0909.32001

[Hörmander 1965] L. Hörmander, " $L^{2}$ estimates and existence theorems for the $\bar{\partial}$ operator", Acta Math. 113 (1965), 89-152. MR 31 \#3691 Zbl 0158.11002

[Kerzman 1972] N. Kerzman, "The Bergman kernel function: Differentiability at the boundary", Math. Ann. 195 (1972), 149-158. MR 45 \#3762

[Kohn 1963] J. J. Kohn, "Harmonic integrals on strongly pseudo-convex manifolds, I", Ann. of Math. (2) 78 (1963), 112-148. MR 27 \#2999 Zbl 0161.09302

[Kohn 1964] J. J. Kohn, "Harmonic integrals on strongly pseudo-convex manifolds, II", Ann. of Math. (2) 79 (1964), 450-472. MR 34 \#8010 Zbl 0178.11305

[Kohn 1979] J. J. Kohn, "Subellipticity of the $\bar{\partial}$-Neumann problem on pseudo-convex domains: Sufficient conditions", Acta Math. 142:1-2 (1979), 79-122. MR 80d:32020 Zbl 0395.35069

[Krantz 1992] S. G. Krantz, Function theory of several complex variables, 2nd ed., Wadsworth \& Brooks/Cole, Pacific Grove, CA, 1992. MR 93c:32001 Zbl 0776.32001

[Kusuoka and Stroock 1985] S. Kusuoka and D. Stroock, "Applications of the Malliavin calculus, II”, J. Fac. Sci. Univ. Tokyo Sect. IA Math. 32:1 (1985), 1-76. MR 86k:60100b Zbl 0568.60059

[Nagel 1986] A. Nagel, "Vector fields and nonisotropic metrics", pp. 241-306 in Beijing lectures in harmonic analysis (Beijing, 1984), edited by E. M. Stein, Ann. of Math. Stud. 112, Princeton Univ. Press, 1986. MR 88f:42045 Zbl 0607.35011

[Nagel et al. 1988] A. Nagel, J.-P. Rosay, E. M. Stein, and S. Wainger, "Estimates for the Bergman and Szegő kernels in certain weakly pseudoconvex domains", Bull. Amer. Math. Soc. (N.S.) 18:1 (1988), 55-59. MR 89a:32025 Zbl 0642.32014

[Nagel et al. 1989] A. Nagel, J.-P. Rosay, E. M. Stein, and S. Wainger, "Estimates for the Bergman and Szegó kernels in $\mathbb{C}^{2}$, Ann. of Math. (2) 129 (1989), 113-149. MR 90g:32028 Zbl 0667.32016

[Ohsawa 1984] T. Ohsawa, "Boundary behavior of the Bergman kernel function on pseudoconvex domains”, Publ. Res. Inst. Math. Sci. 20:5 (1984), 897-902. MR 86d:32025 Zbl 0569.32013 
[Pflug 1975] P. Pflug, "Quadratintegrable holomorphe Funktionen und die Serre-Vermutung", Math. Ann. 216:3 (1975), 285-288. MR 52 \#3599 Zbl 0294.32009

[Pflug 1982] P. Pflug, "Various applications of the existence of well growing holomorphic functions", pp. 391-412 in Functional analysis, holomorphy and approximation theory (Rio de Janeiro, 1980), edited by J. A. Barroso, North-Holland Math. Stud. 71, North-Holland, Amsterdam, 1982. MR 85b:32018 Zbl 0501.32009

[Stein 1993] E. M. Stein, Harmonic analysis: Real-variable methods, orthogonality, and oscillatory integrals, Princeton Mathematical Series 43, Princeton University Press, 1993. MR 95c:42002 Zbl 0821.42001

[Stein and Weiss 1971] E. M. Stein and G. Weiss, Introduction to Fourier analysis on Euclidean spaces, Princeton Mathematical Series 32, Princeton University Press, 1971. MR 46 \#4102 Zbl 0232.42007

Received March 1, 2009.

JENNIFER HALFPAP

DEPARTMENT OF MATHEMATICAL SCIENCES

UNIVERSITY OF MONTANA

MisSOULA, MT 59812

UNITED STATES

halfpap@mso.umt.edu

http://www.umt.edu/math/People/Halfpap.html

AleXANDER NAGEL

DEPARTMENT OF MATHEMATICS

UNIVERSITY OF WISCONSIN

480 LINCOLN DRIVE

MADISON, WI 53706-1388

UNITED STATES

nagel@math.wisc.edu

http://www.math.edu/ nagel

STEPHEN WAINGER

DEPARTMENT OF MATHEMATICS

UNIVERSITY OF WISCONSIN

480 LINCOLN DRIVE

MADISON, WI 53706-1388

UNITED STATES

wainger@math.wisc.edu 